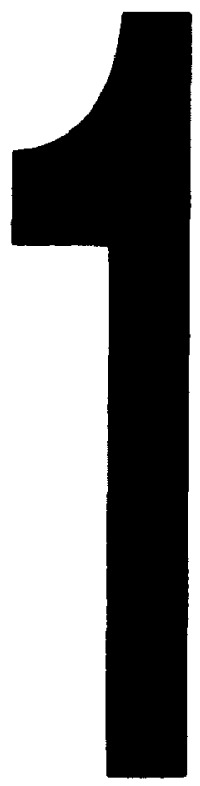

PM-1 3\%"X4" PHOTOGRAPHIC MICROCOPY TARGET NBS 1010a ANSI/ISO \#2 EOUIVALENT

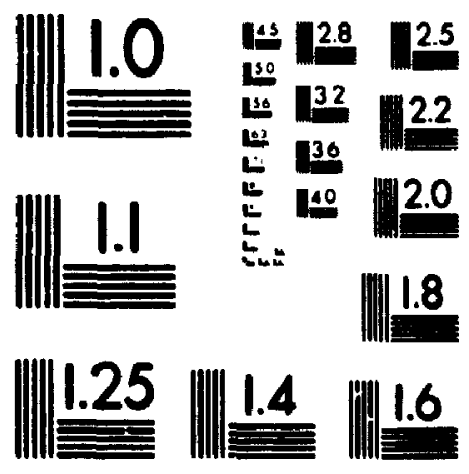

PRECISION ${ }^{\text {MW }}$ RESOLUTION TARGETS 


\title{
PERFORMANCE OF LOAD SHARING POLICIES IN \\ HOMOGENEOUS AND HETEROGENEOUS SYSTEMS
}

\author{
by \\ KWOK CHEONG MICHAEL LO, B.C.S. \\ A thesis submitted to \\ the Faculty of Graduate Studies and Research \\ in partial fulfillment of the requirements for the degree of
}

Master of Computer Science

Ottawa-Carleton Institute for Computer Science

School of Computer Science

Carleton University

Ottawa, Ontario

April 15, 1996

○ copyright

1996, Kwok Cheong Michael Lo 
National Library

of Canada

Acquisitions and

Bibliographic Services Branch

395 Wellington Street

Ottawa. Ontario

KIA ON4
Bibliotheque nationale

du Canada

Direction des acquisitions et

des services bibliographiques

395. rue Wellington

Ottawa (Ontario)

K1A ON4

Vour ile votre ioliesence

Our the Notre ifference

The author has granted an irrevocable non-exclusive licence allowing the National Library of Canada to reproduce, loan, distribute or sell copies of his/her thesis by any means and in any form or format, making this thesis available to interested persons.
L'auteur a accordé une licence irrévocable et non exclusive permettant à la Bibliothèque nationale du Canada de reproduire, prêter, distribuer ou vendre des copies de sa thèse de quelque manière et sous quelque forme que ce soit pour mettre des exemplaires de cette thèse à la disposition des personnes intéressées.

L'auteur conserve la propriété du droit d'auteurr qui protège sa thèse. Ni la thèse ni des extraits substantiels de celle-ci ne doivent être imprimés ou autrement reproduits sans son autorisation.
The author retains ownership of the copyright in his/her thesis. Neither the thesis nor substantial extracts from it may be printed or otherwise reproduced without his/her permission. 
Nomo Kwork Cheore Michnel lo

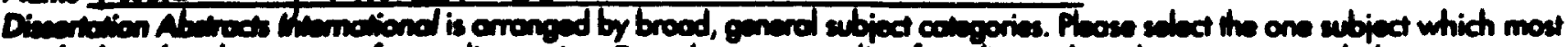

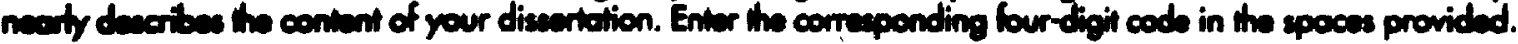

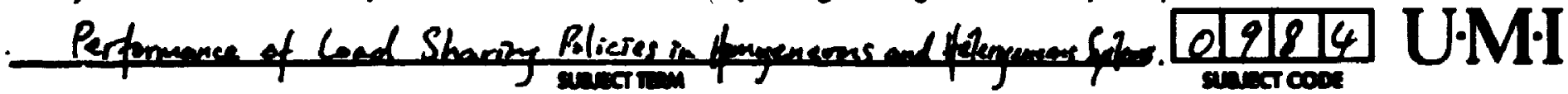

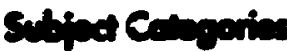

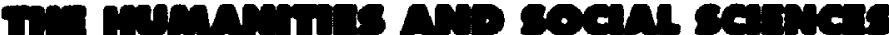

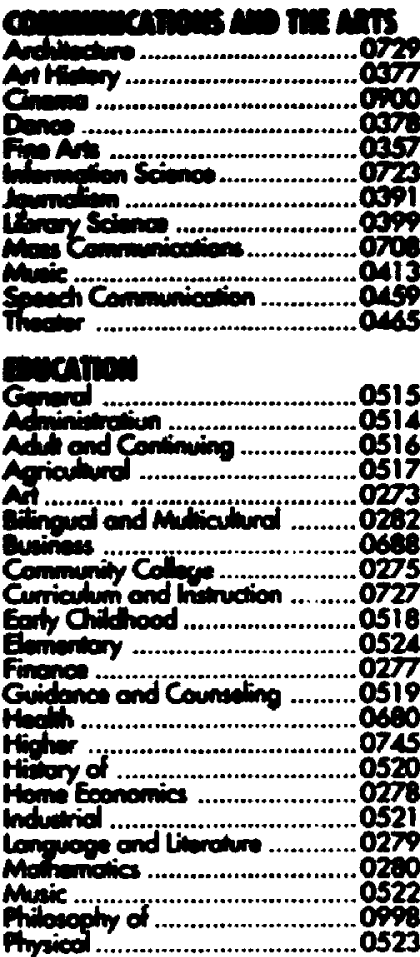

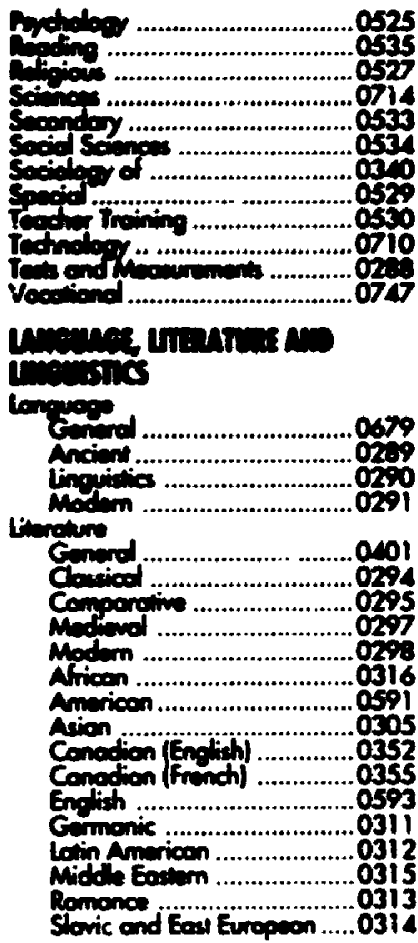

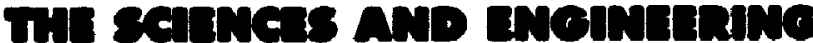

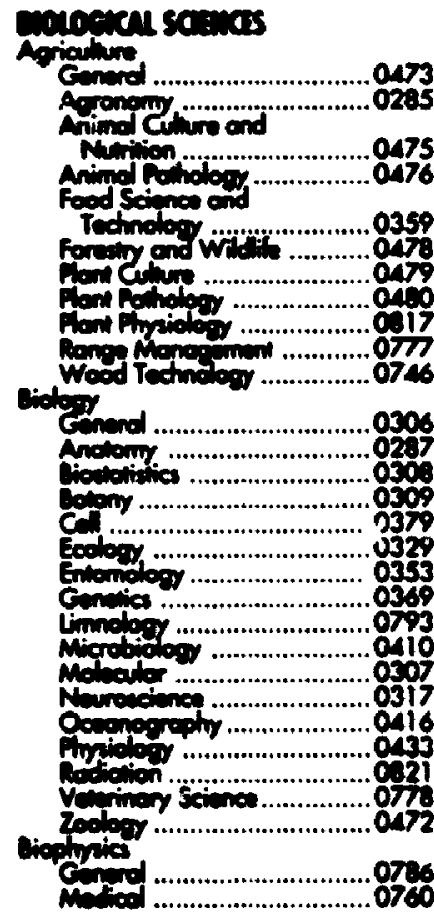

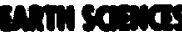

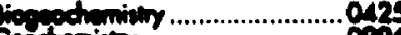

Godery ....................................0370 Gology ...................................037 Hor

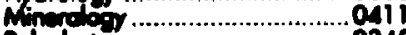

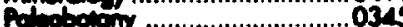

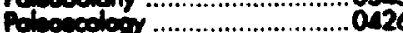

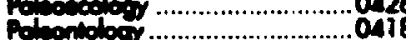

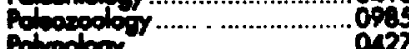

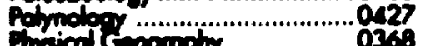
Pinpiced Ocoinegrepty ........... .0415

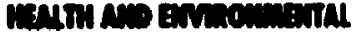
scinas

Envenmentel Sciences .............0768 Hoinh Sciences:

Gomed ............................0566

Autioley. ...........................030

Chenelverap .................. Opp

Dentistry …..........................056

Edvedion ...........................0350

Howiol Monocornent ...........0769

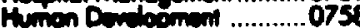

monolo.

Medicine ond Sureny ..........058

Mented Hodis .....................034

Nursing ..............................0568

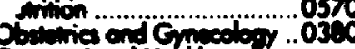

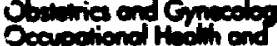

Therey .............................035

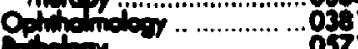

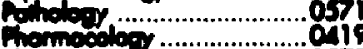

promed ............................057

promed fi.......................8d

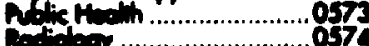

Radied ...........................0575

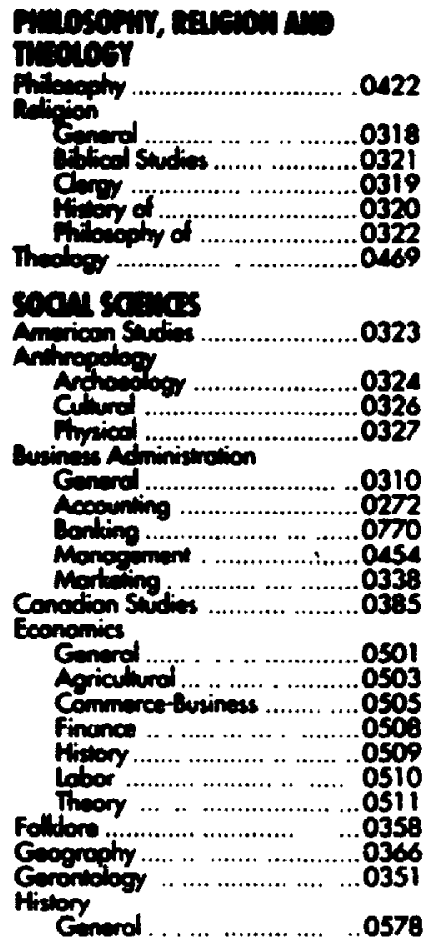

Aximt .................. . $\mathbf{0 5 7 9}$

Madion ...................... go

Modom .....................

Aco......................... gost

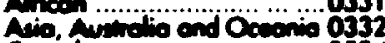

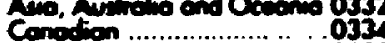

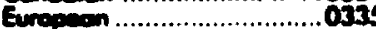

Letip Anorioen .....................g33

pongen

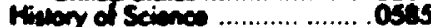

Low ore

Peliniool science

Gonerd...............

0615

portions.................

0816

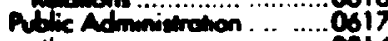

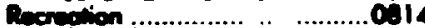

Socid Work ..............................0452

Sociology

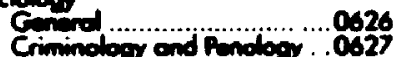

o93

Etric ond locied sudien $\quad 0631$

Individual and fomily

062

Induetid ond iobor

Relotion ....... 062

Public and socid watione ....0830

Sociod sencture and

Domosprent ............ 0700

Theory and Muhods .... .... 034

Tromportation ......

Women's sudies .. .. .... .0453
Engineming

Anowed.

Arrieculured.

Aurometive

Bionedicel

Chemeal

os.3

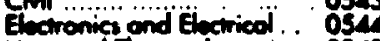

Heat and Themodmanici 034

Axdralic..................... 054

modutried

Marim

Moriots Science......

Mectuonicel.. . . . ...... 0540

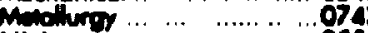

Mining.

Nuebor

Podkoging

Potroleng

Gowetmology... .042

Operetions krowach ...

Tautib Technolooy. ... ... 099

crepust

Emavione

Chinicoil

Dombonenter

Eeponimantel

Indentive

Prometion

Anpiobicos

rectebled

Socie

0621

36.

0.22

(2)

0.23

cotd san ........ Os

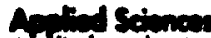

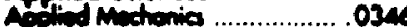

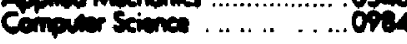


The undersigned recommend to the Faculty of Graduate Studies

and Research acceptance of the thesis

\title{
"PERFORMANCE OF LOAD SHARING POLICIES IN HOMOGENEOUS AND HETEROGENEOUS SYSTEMS"
}

\author{
submitted by \\ Kwok Cheong Michael Lo, B.C.S. \\ in partial fulfiliment of the requirements for \\ the degree of Master of Computer Science
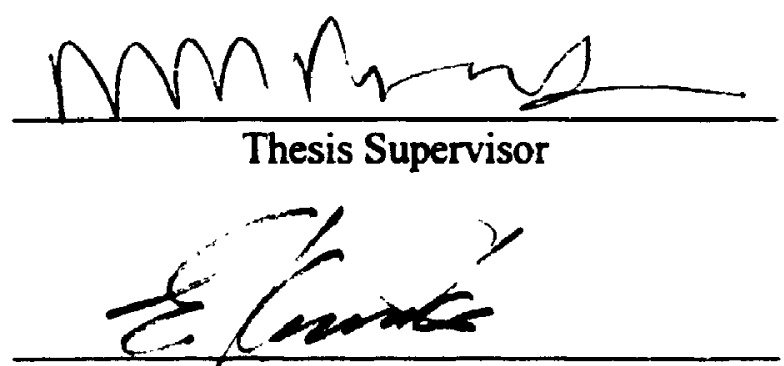

- Director, School of Computer

Carleton University

May 16, 1996 


\begin{abstract}
Load sharing improves the performance of distributed systems by transferring jobs from highly loaded computers to lightly loaded computers. Most of the previous studies have evaluated load sharing policies only in a homogeneous system model. Furthermore, some good load sharing policies have not been compared under the same system and workload models.
\end{abstract}

This thesis compares the performance of six load sharing policies in a homogeneous system model and the performance of five load sharing policies in two types of heterogeneous models. These policies are the sender-initiated policy, the receiver-initiated policy, the periodic symmetrically-initiated policy, the stable symmetrically-initiated adaptive policy, the hierarchical policy and the single-coordinator policy. The periodic symmetrically-initiated policy is not considered in the heterogeneous models as its performance is substantially worse than that of the stable symmetrically-initiated adaptive policy in the homogeneous system model.

The results indicate that the single coordinator policy, the stable symmetricallyinitiated adaptive policy and the hierarchical policy are the best three policies among the six policies considered in this thesis. 


\section{ACKNOWLEDGEMENTS}

I would like to express my deepest appreciation to my thesis supervisor, Dr. S. Dandamudi, for his continued interest and guidance provided during the development of this thesis and throughout my study at Carleton University. Finally. I would like to present this thesis to my beloved parents and brother. 


\section{TABLE OF CONTENTS}

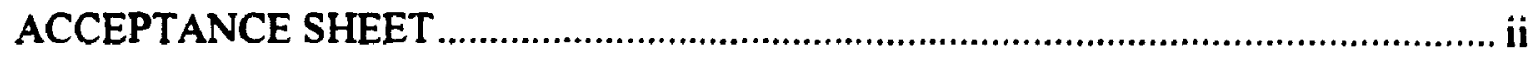

ABSTRACT

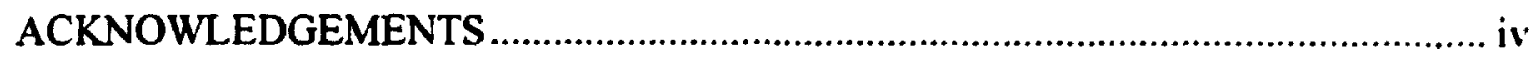

TABLE OF CONTENTS

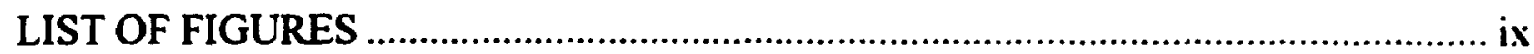

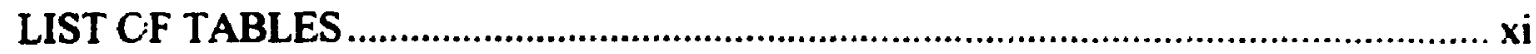

\section{CHAPTER I}

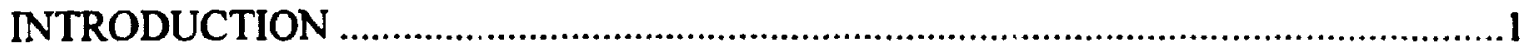

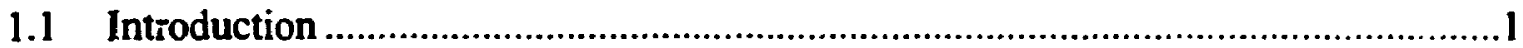

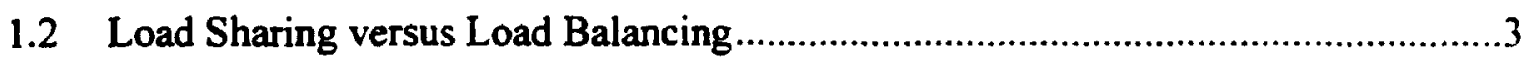

1.3 Issues in Load Sharing ....................................................................................

1.3.1 Classes of Load Sharing Policies .................................................................

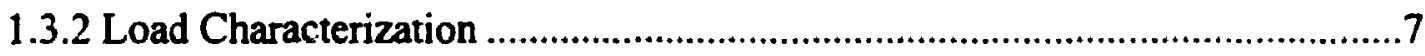

1.3.3 Components of Load Sharing Policies ...........................................................

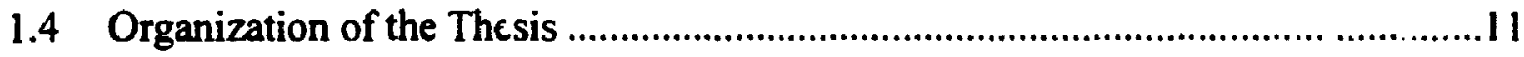

\section{CHAPTER II}

A SURVEY OF DYNAMIC LOAD SHARING POLICIES ……................................13

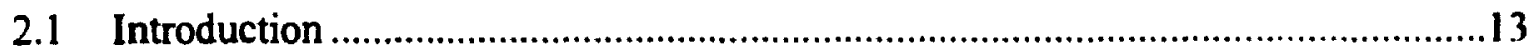

2.2 The State Broadcast Algorithm (STB) ……...................................................13

2.3 The Broadcast Idle Algorithm (BID) ………....................................................15

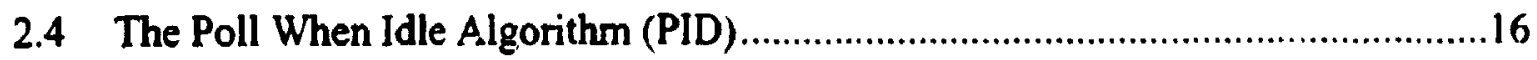




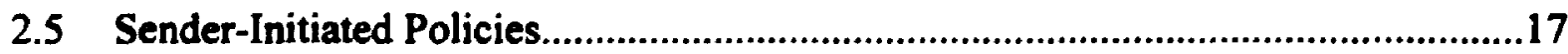

2.5.1 Random Sender-Initiated Policy (RSI) ....................................................18

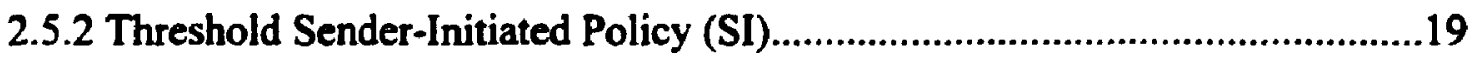

2.5.3 Shortest Sender-Initiated Policy (SSI) ........................................................20

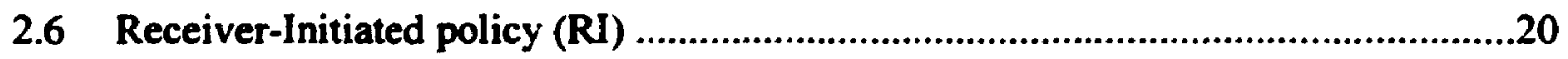

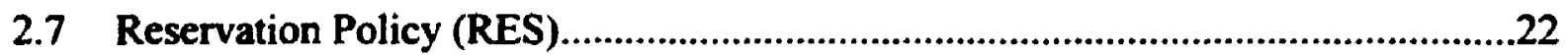

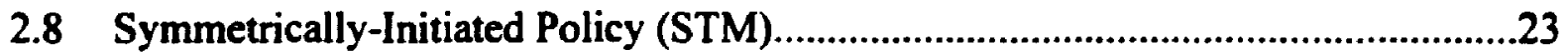

2.9 Stable Symmetrically Initiated Adaptive Policy (SSYM)........................................24

2.10 Stable Sender-Initiated Adaptive Policy (SSIA) ....................................................28

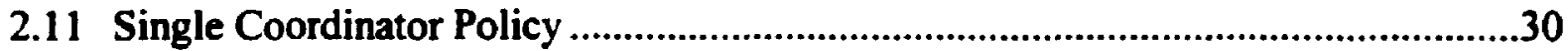

2.12 Periodic Symmetrically-Initiated Policy (PSI) ...........................................................31

2.13 Hierarchical Receiver-Initiated Policy (HRI) ............................................................32

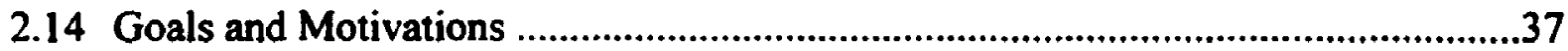

CHAPTER III

PERFORMANCE OF LOAD SHARING POLICIES IN A

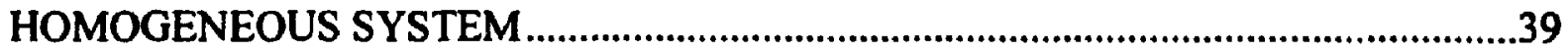

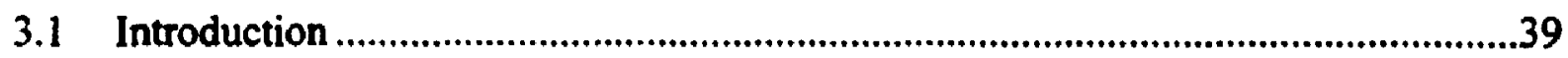

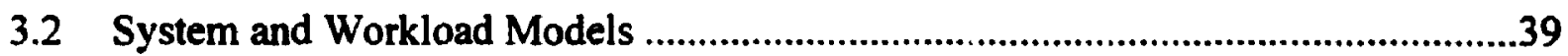

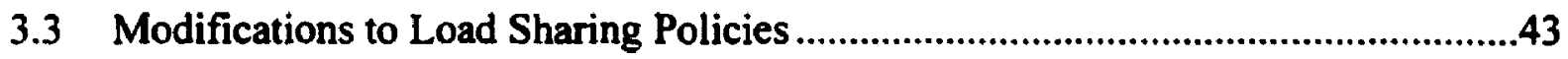

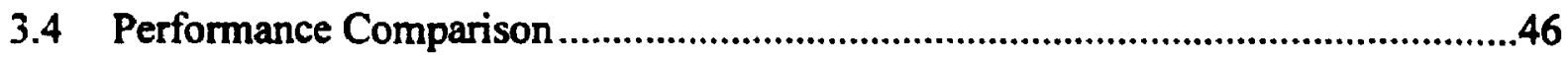

3.4.1 Performance as a Function of System Load ........................................................47

3.4.2 Sensitivity to Variance in Inter-Arrival Times..............................................55

3.4.3 Sensitivity to Variance in Service Times .......................................................57

3.4.4 Performance Sensitivity to Probe Limit .............................................................59 
3.5 Two Variations of the Hierarchical Receiver-Initiated Policies.

3.5.1 Global Hierarchical Load Sharing Policies

3.6 Performance Comparison of Hierarchical Load Sharing Policies .64

3.6.1 Principal Performance Comparison 64

3.6.2 Sensitivity to Variance in Service Times and Inter-Arrival Times 67

3.6.3 Performance Sensitivity to Branching Factor .69

3.6.4 Reduction in Message Handling Rate 72

3.7 Summary .77

\section{CHAPTER IV}

PERFORMANCE OF LOAD SHARING POLICIES IN HETEROGENEOUS SYSTEMS .80

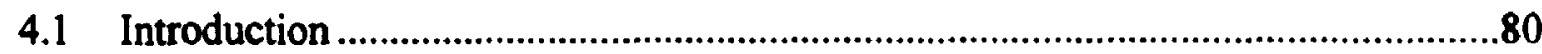

4.2 System and Workload Models ....................................................................

4.3 Performance Comparison of Five Load Sharing Policies in Type I Systems .........81

4.3.1 Mean Response Time as a Function of System Load ...............................83

4.3.2 Sensitivity to the Inter-Arrival Time Coefficient of Variation......................87

4.3.3 Performance Sensitivity to Variance in Service Times...............................89

4.3.4 Sensitivitv to the Degree of Heterogeneity ..............................................91

4.4 Performance of Five Load Sharing Policies in Type II Systems .......................93

4.4.1 Performance as a Function of System Load ..............................................93

4.4.2 Impact of the Degree of Heterogeneity ..................................................95

4.5 Performance of Hierarchical Policies in Type I Heterogeneous Systems ...............97

4.5.1 Performance as a Function of Offered System Load on Class II Nodes .........97

4.5.2 Sensitivity to Variance in Inter-Arrival Times......................................... 100 
4.5.3 Sensitivity to Variance in Service Times ....................................................101

4.5.4 Impact of the Degree of Heterogeneity .........................................................102

4.6 Performance of Hierarchical Policies in Type II Heterogeneous Systems.............104

4.6.1 Performance as a Function of Offered Load ...............................................104

4.6.2 Performance Sensitivity to the Degree of Heterogeneity ..............................105

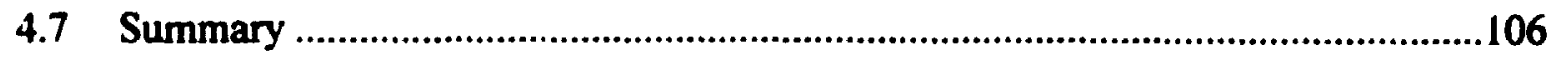

CHAPTER V

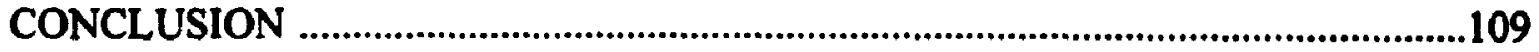

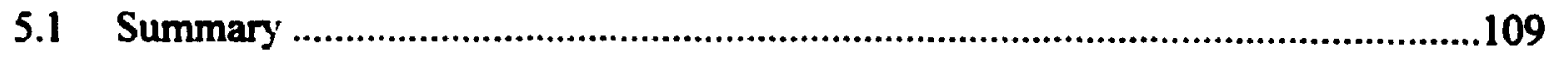

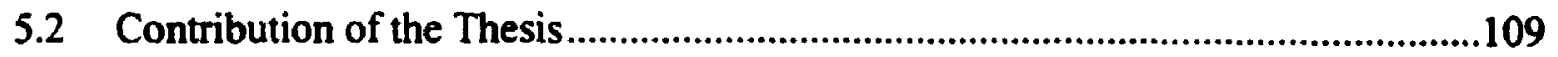

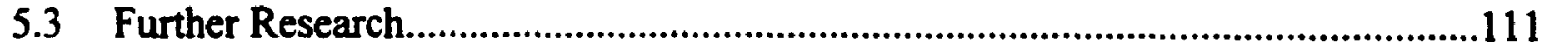

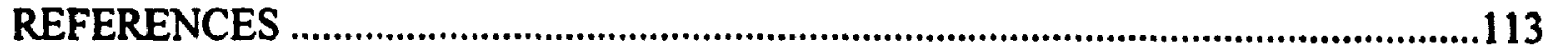




\section{LIST OF FIGURES}

Figure 1.1 A load imbalance distributed computer system .......................................2

Figure 2.1 An example of a local hierarchical organization for an 8-node system with a branching of 2 . .........................................................................................33

Figure 3.1 A system model of a typical distributed computer system ........................41

Figure 3.2(a) Mean response time as a function of offered load .....................................48

Figure 3.2(b) Mean response time as a function of offered load (RI, R-RI, SI, LHRI)...50

Figure 3.3 Mean response time as a function of offered load ......................................53

Figure 3.4 Performance sensitivity to inter-arrival coefficient of variation (CV).......56

Figure 3.5 Performance sensitivity to service time coefficient of variation................57

Figure 3.6 Performance sensitivity to probe limit........................................................60

Figure 3.7 An example of global hierarchical organization for an 8-node system with branching factor $\mathrm{B}=2$

Figure 3.8(a) Mean response time versus offered system load.......................................65

Figure 3.8(b) Mean response time versus offered system load......................................67

Figure $3.9 \quad$ Impact of variance in service times..........................................................68

Figure 3.10 Performance sensitivity to inter-arrival CV .............................................69

Figure 3.11 Performance impact of branching factor on three hierarchical policies.....70

Figure 3.12(a) Average message handling rate at leaf level .............................................74

Figure 3.12(b) Average message handling rate at middle level......................................74

Figure 3.12(c) Average message handling rate at root level ............................................75

Figure 3.13(a) Average message handling rate at leaf level ..............................................75

Figure 3.13(b) Average message handling at middle level.................................................76

Figure 3.13(c) Average message handling rate at root level .........................................76 
Figure 4.1(a) Mean response time as a function of offered load on Class II nodes.........83

Figure 4.1(b) Mean Response time as a function of offered system load on Class II nodes.

Figure 4.2 Performance sensitivity to inter-arrival CV ..........................................87

Figure 4.3 Impact of variance in service times.............................................................89

Figure 4.4 Impact of the degree of heterogeneity........................................................91

Figure 4.5 Performance sensitivity to offered system load .........................................94

Figure 4.6 Mean response time as a function of \# of class I nodes.............................96

Figure 4.7(a) Performance as a function of system load on Class II nodes.....................98

Figure 4.7(b) Perfonnance as a function of Offered load on Class II nodes...................99

Figure 4.8 Performance sensitivity to inter-arrival $\mathrm{CV}$...............................................100

Figure 4.9 Performance sensitivity to service time CV .........................................102

Figure 4.10 Performance sensitivity to the degree of heterogeneity ...........................103

Figure 4.11 Performance as a function of system load ..................................................104

Figure 4.12 Performance sensitivity to degree of heterogeneity ...................................106 


\section{LIST OF TABLES}

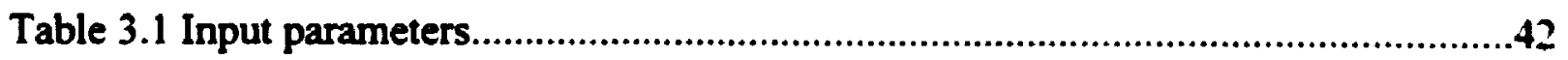




\section{CHAPTER I \\ INTRODUCTION}

\subsection{Introduction}

A distributed computing system consists of a collection of autonomous computers linked by a computer network and equipped with a set of software modules which enable the interconnected computers to share resources and coordinate activities. The computers in a distributed computer system will be referred to as nodes. Jobs' are usually generated independently and randomly at each node in a distributed system. In such a system some nodes may have more jobs than others to process (see Figure 1.1). This situation is referred to as load imbalance. Livny and Melman [Liv82] have shown that, in a homogeneous distributed computer system in which all nodes are identical, there is a high probability that the distributed computer system is in a state where at least one job is waiting for service and at least one node is idle. This load imbalance degrades overall system performance as computing resources are not fully utilized. Load sharing reduces the level of load imbalance by transparently transferring jobs from heavily loaded nodes to lightly loaded or idle nodes, in $\mathrm{c}$. Cer to improve the overall system performance. Load sharing requires a control policy and a job placement mechanism. While a job placement mechanism provides efficient means to actually transfer a job from one node to another

\footnotetext{
Jobs, tasks and processes are regarded as execution units. "Job", "task", and "process" are used interchangeably in this context.
} 
node, a control policy decides when, where and which jobs are transferred, such that the intended benefit is achieved. This thesis concentrates on the performance of load sharing policies rather than job placement mechanisms. An example of job placement mechanism and process migration can be found in [Zho93] and [Dou91] respectively.

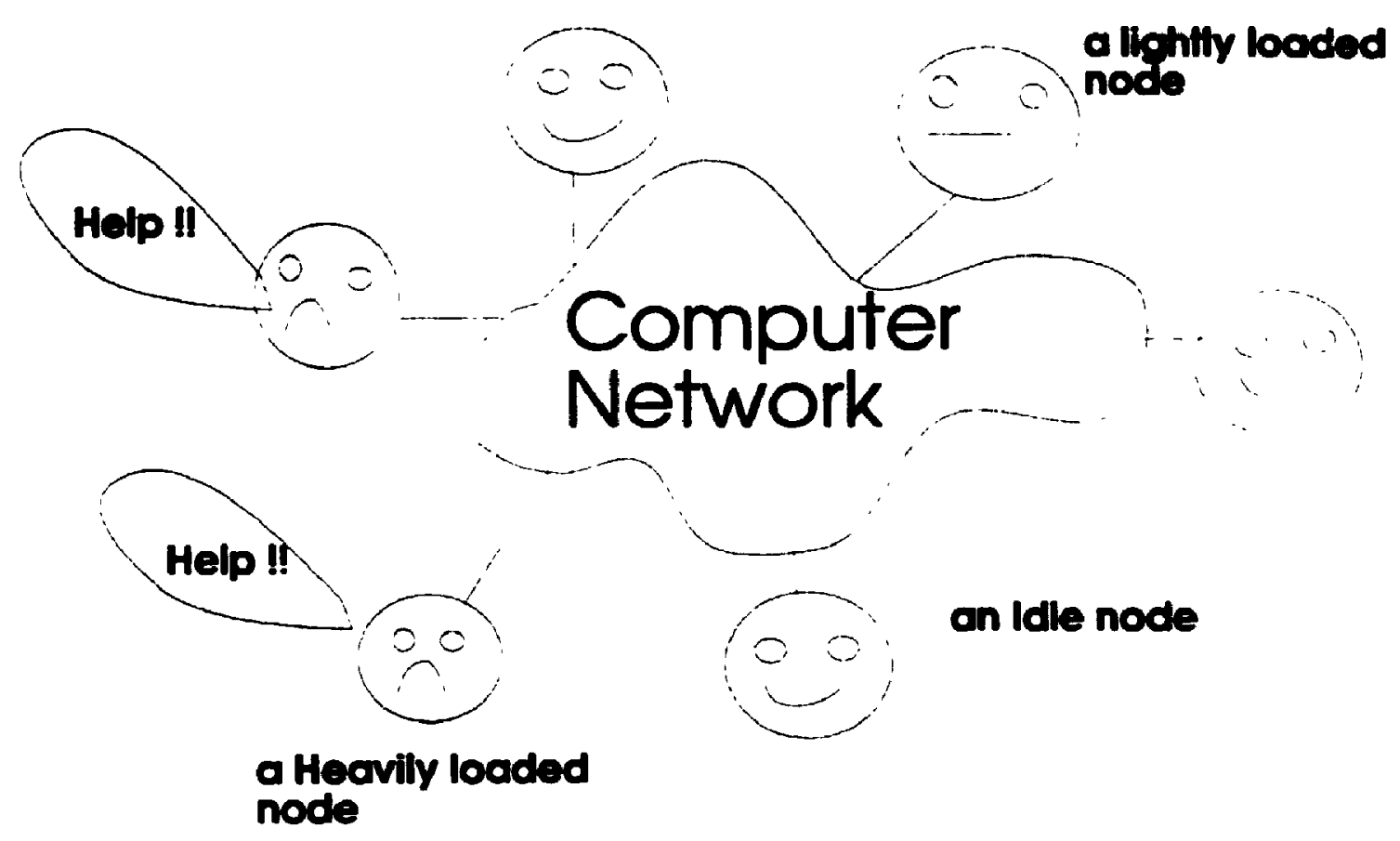

Figure 1.1 A load imbalance distributed computer system.

In examining existing literature, the following three methods have been used to study the performance of load sharing policies: analytical modeling [Eag86a,b]; simulation modeling [Dan95a,b]; and prototyping [Zho93]. The major drawback of the first approach is that most of the analytical models are based on some assumptions that may not be realistic to a system under study. For example, in Eager's model [Eag86a] the 
communication cost is assumed to be negligible. It has been shown [Mir90] that communication cost has an impact on the performance of load sharing policies. Thus, Eager's model was used to compute approximate performance only. Moreover, it has been shown by Wang et al. [Wan85] that even a simple load balancing policy can lead to an intractable mathematical model. Computer simulation represents the best alternative to analytical modeling, since simulation modeling is based on more realistic assumptions. Computer simulation can be divided into two types: trace-driven and self-driven. The first type uses traces as an input to a simulation program. A tace is a stream of events observed in an operational system. The input of the self-driven simulation program is represented by a set of parameters whose values are generated from probability functions with appropriate statistical distributions. Prototyping is sometimes not possible because hardware and software are not often available; and the costs of prototyping might be too high. The results from prototyping could be too specific and can only be applied to a specific type of distributed computing system. Thus, simulation modeling has been chosen to study the performance and characteristics of load sharing policies in this thesis.

\subsection{Load Sharing versus Load Balancing}

There are two types of load distributing policies to reduce load imbalance in distributed computer systems. They are load sharing and load balancing policies. The main difference between load sharing and load balancing policies is regarding the objec:ives of the two types of load distributing policies. Both types of policies attempt to provide 
conservation of work. In other words, they strive to prevent a distributed system from a state in which some computers lie idle while some jobs are waiting for service at some other computers. This state is referred to as an unshared state [Liv82]. However, load balancing not only conserves work but also attempts to equalize the loads at all computers in a distributed system. Under Krueger and Livny's model [Kru87], in which the migration cost is zero, load baiancing results in better process wait time and mean wait ratio than load sharing. Since load balancing attempts to equalize workload, it requires a higher migration rate than load sharing. The overheads associated with higher migration rate may reduce or eliminate the potential benefit of load balancing over load sharing. Load sharing may potentially provide better performance than load balancing when the system load is high.

\subsection{Issues in Load Sharing}

This section is a brief summary of issues in load sharing. An excellent review of the issues in load sharing policies is given in Shivaratri et al. [Shi92]. The terminology used in this section is similar to that in [Shi92].

\subsubsection{Classes of load sharing policies}

Load sharing policies can be broadly classified as static policies, dynamic policies and adaptive policies. A static policy does not take load information into consideration when making load sharing decisions, whereas both dynamic and adaptive policies use load information for such decisions. 
Shivaratri et al. [Shi92] have given two examples of static load sharing policy. The first static load sharing policy is based on a formula. Jobs are remotely or locally executed depending on the output value of the following static formula: $\mathrm{K} \bmod \mathrm{N}$ where $\mathrm{N}$ is the number of nodes in the system and $\mathrm{K}$ is the job number. The second static policy uses probability $P_{j}$ in assigning a job to node $j$ where $P_{j}$ is predetermined statically with system information such as the average task-initiation rate and execution rate for each node. The main advantage of static policies is simplicity. Since static policies do not consider the current system state in choosing transfer partners, it could possibly increase the response time of tasks, but not decrease response time when inappropriate job allocation occurs. In the first example, if job number 2 arrives at a node 1 with a system size of 10 then this job is transferred to node 2. It is an unwise transfer decision if node 2 is overloaded but node 1 is idle. In this case, the response time of job 2 would be higher than if the job could be executed locally. Similarly, the second example of static policy could also lead to a wrong assignment of jobs to nodes.

Unlike static policies, dynamic policies use load information to make load sharing decisions in order to avoid incorrect selections of transfer partners. One example that contrasts to the above static policies might be checking the local load information before transferring jobs. This would avoid the problem of transferring tasks from an idle node to a 
busy node. In Chapter 2 a survey of dynamic algorithms will be presented to give more details on dynamic algorithms?.

The next class of policies is the adaptive policy, which is actually a specialization of the dynamic policy. Adaptive policies disseminate. acquire and manipulate load information and they make decisions based on the information acquired. As well they change their parameter values and sub-policies according to the load information obtained. For instance, an adaptive policy might change its load sharing threshold value ${ }^{3}$ and subpolicies according to overall system utilization. Some sub-policies may perform better in some range of system loads while others may perform better at another spectrum of system loads.

Both dynamic and adaptive load sharing policies incur more overheads and are more complex than static load sharing policies. If these overheads are kept minimal, dynamic and adaptive policies have the potential of outperforming static load sharing policies by better avoidance of wrong jobs allocations. Nevertheless, it has been shown [Eag86b] that simple dynamic load sharing policies provide considerable performance improvement over no load sharing.

\footnotetext{
${ }^{2}$ Algorithm and policy are used interchangeably in this thesis

${ }^{3}$ In the literature, threshold values are static.
} 


\subsubsection{Load Characterization}

The choice of load index has a substantial impact on load sharing policies. Workloads are normally characterized by some load index such as: the run queue length; the mean CPU queue length over some period; the percentage of idle CPU-time; the amount of unused memory; the average context-switch rate; and the average system call rate. Knuz [Knu91] demonstrated that the performance of a heuristic load sharing policy is affected by the choice of workload index. He has also shown that the most effective load index is the CPU queve length and that even the combinations of all the above mentioned indexes cannot excel this simple load index. In fact, the CPU job queue length is usually the choice of most performance studies of load sharing policies in the literature.

\subsubsection{Components of Load Sharing Policies}

Dynamic load sharing policy generally consists of four sub-policies: a transfer policy, a location policy, a selection policy and an information policy.

A transfer policy determines whether a particular node is suitable for load sharing activities based on its load index. A common type of transfer policy is the threshold transfer policy. Under this transfer policy, current load index is checked against static threshold(s). In a hierarchical policy, two thresholds are used to identify the state of nodes [Dan96]. 
In the one threshold case, if the load index of a node is higher than the static threshold, say $T$, then the node is regarded as a sender. An overloaded node is a sender in the sense that it has a job to send out. On the other hand, if the load index is less than $T$. then it is regarded as a receiver. Similarly, a lightly loaded node is a receiver because it attempts to acquire a job from another node. A node is regarded as an OK node if it is neither a sender nor a receiver node. An OK node does not need to participate in load sharing.

In the two thresholds case, say $T_{h}$ and $T_{l}, T_{h}$ is the upper threshold and $T_{1}$ is the lower threshold. If the load index of a node is higher than $T_{h}$ then it is identified as a sender, whereas if the load index of a node is less than $T_{1}$ then it is a receiver. If the load index is greater that $T_{1}$ but less than or equal to $T_{h}$, then it is an $O K$ node. The use of two threshold values allow us to increase the difference . if necessary, between a serder node and a receiver node in terms of load index. An important condition for $T_{h}$ and $T_{1}$ is that $T_{h}$ must be greater than or equal to $T_{1}$. Note that in some studies, the location policy and the transfer policy use the same threshold(s) to make their decisions but in other studies they use different threshold to make their decisions. However, this difference does not change the basic semantics of the load sharing policies under studied.

The selection of the threshold value has a great influence on the performance of load sharing policies as shown in [Ben94], [MIR89] and [Dan95a]. Briefly, in a load sharing policy with one threshold, the mean response time is an increasing function of threshold 
value after an optimal value is reached for some policies because increasing the threshold value decreases the scope of load sharing. A system is a no-load sharing system when the value of the threshold of the transfer policy becomes infinity. For very high values of threshold, at any time the job queue length or some other load index never reaches the threshold. In other words, all nodes are not senders. However, setting the threshold at a very low value (e.g., $T=1$ ) may not be suitable for some policies (e.g., the sender-initiated policies). This is because most of the time the load index of any node is above that value at high system loads that all nodes are senders. In both cases, a transfer partner cannot be selected. Moreover, the optimal value of the threshold of a policy may be different for different levels of system utilization [Ben94] and communication delays [Mir89]. Similarly, a policy with two thresholds should set the value of the two thresholds according to the policy characteristics, the levels of system utilization and communication delays.

A location policy is responsible to find a suitable transfer partner for a node when it has been identified as a sender or recerver by the transfer policy. The location policy works closely with the information and transfer policy. Its decision is based on the load information collected by the information policy and sometimes the rule set out by the transfer policy. It can be broadly classified into two types: the centralized location policy and the distributed location policy. In the distributed location policy, each node finds its own transfer partner(s). It also decides which node to be polled, in an attempt to select a suitable load sharing partner. Polling is a common method for acquiring load information 
and can be done in serial or parallel (e.g., multicast) fashion. The selection of which node to be polled can be made on a random basis [Eag86b] or on previously polled information [Shi90]. In a centralized policy, a single node, called a coordinator, keeps the load information of all nodes collected via information policy. The coordinator is consulted by all the nodes that need a transfer partner. In other words, this coordinator makes all transfer decisions.

A selection policy chooses which jobs to be transferred when the location policy finds a suitable partner. The two major types of selection policies are preemptive and nonpreemptive selection policies. A preemptive selection policy involves both preemptive and non-preemptive jr transfers but a non-preemptive selection policy solely involves nonpreemptive job transfers. A preemptive job transfer moves partly executed jobs between two nodes, whereas a non-preemptive job transfer moves only newly arrived jobs (i.e., jobs have not been executed yet) from one node to another node. The preemptive transfer is also called process migration. Transferring partly executed jobs requires transferring the state of the job during execution. The state includes virtual memory, open file descriptors, pending messages, signals, etc. On the other hand, a non-preemptive job transfer only requires to transfer the binary file of the job. Since the size of a binary file is relatively smaller than that of the process state information, the cost for transferring a new job is relatively cheaper than that for process migration. It has been shown [Eag88] that there is only limited 
performance benefits to migrating active processes under some conditions. For these reasons, many proposed load sharing policies only allow non-preemptive job transfers.

An information policy is responsible for determining when the load information of other nodes in a system is collected. Information policies can be divided into three types: the periodic policy, the state-driven policy and the demand-driven policy. In the periodic policies every node in the system will disseminate (collect) load information to (from) a subset of nodes at the end of a preset time period. In the state-driven policy, nodes will only notify a subset of nodes, if and only if, when their state has changed (e.g., from a lightly loaded node to a heavily loaded node). A demand-driven policy is a policy in which a node acquires load information from other nodes only when it needs to. That is, when the transfer policy decides that the node is a sender or receiver and the location has selected a particular node for polling or probing.

\subsection{Organization of the Thesis}

This thesis is a performance study of load sharing policies in homogeneous distributed systems and in two types of heterogeneous distributed systems. As well, a survey of load sharing policies is also given. The results of the simulation experiments on the load sharing policies is presented in two chapters, one for each type of distributed systems. Finally, a summary of the results is presented. 
The remainder of this thesis is organized as follows. Chapter 2 presents a survey of dynamic load sharing policies. At the end of the chapter, reasons for choosing some policies over other policies for performance analysis in the thesis.

Chapter 3 describes the system and workload models used in the simulation experiments for homogeneous distributed system. It then provides a necessary extension of the selected policies to fit into the simulation model described. It presents the results of simulation experiments for six load sharing policies for a homogeneous distributed system. The last section of this chapter summarizes the findings of the experiments.

The first part of Chapter 4 describes the system and workload models used for heterogeneous distributed systems. It then describes the results of the simulation study of the performance regarding load sharing policies for Type I and Type II heterogeneous systems. In a heterogeneous system, nodes may not be functionally identical, be subject to different arrival rates of jobs, and have different processing rates. In the literature [MIR90], heterogeneous systems are classified into two types: Type I and Type II. In Type I heterogeneous distributed systems, nodes are functionally identical but the job arrival rates at nodes are different. In Type II heterogeneous distributed systems, nodes may have different processing rates and may also be subjected to different arrival rates of jobs. Finally, Chapter 5 presents a summary of this thesis. The contributions of this thesis and directions for further research are also given in Chapter 5 . 


\section{CHAPTER II}

\section{A SURVEY OF DYNAMIC LOAD SHARING POLICIES}

\subsection{Introduction}

This chapter presents a survey of ioad sharing policies studied in the literature. They are described in terms of sub-policies: the transfer policy; the location policy; the information policy; and the selection policy. Six load sharing policies, namely the sender-initiated (SI), receiver-initiated (RJ), periodic symmetrically-initiated (PSI), stable symmetrically-initiated (SSYM), local hierarchical receiver-initiated (LHRI) and single coordinator (SINCO) policies, are chosen for performance analysis in a homogeneous system model. Following this analysis five of the six policies, SI, RI, SSYM, LHRI and SINCO, are chosen for performance evaluation in heterogeneous system models. The motivation and the goals of this thesis are also presented in this chaprer.

\subsection{The State Broadcast Algorithm (STB)}

The state broadcast algorithm, the broadcast idle algorithm and the poll when idle algorithm were proposed by Livny and Melman in [Liv82]. Section 2.3 and 2.4 will present the latter two algorithms. All three algorithms assume that the communication system of a distributed system has message broadcast and uniform distance properties.

The term "uniform distance" means that the expected time that is required to transfer a message from one node to another is the same for all pairs of nodes. In such a system, all 
nodes can be an equal-priority candidate for receiving a job and the only consideration of a load sharing policy is the relative load of the nodes. Message broadcast refers to a facility for sending messages to all of the computers in a given network environment. The message broadcast capability improves the ability of the algorithm to get a global and updated description of system status. The authors also define the following terms: ST, SSV, UBF and BT. Both ST and SSV contain the states of all nodes in the system. The ST is a tuple, $\left(n_{1}, \ldots n_{i}, \ldots, n_{N}\right)$ where $n_{i}$ is the number of tasks in node $i$, that describes the state of the system whereas SSV $=\left(s_{1}, \ldots s_{n}\right)$ is an updated copy of the system state vector. The vector is maintained by the information policy. The state of the system is defined as unbalanced if there are two servers, $i$ and $j$. such that $n_{j}-n_{j}>1$. The unbalanced factor UBF of a state ST is defined as: maximum of $\left(n_{i}-n_{j}\right) / n_{j}$ for $i>0$ and $j \leq N$ if ST is unbalanced and 0 otherwise.

The information policy of the state broadcast algorithm uses the message broadcast and the uniform distance properties of the communication system. Whenever the state of a node changes, due to an arrival or a departure of a job, the node broadcasts a status message that describes its new state. The information policy ensures that each node has an updated and consistent copy of the system state vector (SSV). Note that state vectors (ST) may be different from SSV due to the communication delays. 
The selection policy of STB permits only non-preemptive job transfers. The control elements (transfer and location policies) will transfer a waiting task from node i to node $j$ if the following conditions are fulfilled [Liv82]:

1. $S_{i}-S_{j}>1+\left(B T . S_{j}\right)$ where BT is a parameter that controls the balancing threshold of the algorithm.

2. $\left(S_{i}>S_{k}\right)$ or $\left(S_{i}=S_{k}\right.$ and $\left.i>=k\right)$ for all $k=1, \ldots, N$.

3. $S_{j}<=S_{k}$ for $k=1, \ldots, N$

When more than one node has a minimal number of waiting tasks the selection of the destination node is made randomly.

\subsection{The Broadcast Idle Algorithm (BID)}

Unlike the information policy of STB, the information policy of the broadcast idle algorithm (BID) uses status broadcast but a status message is broadcasted only when a node becomes idle. Each status message alerts all the other nodes and causes them to activate the control element (the transfer policy and the location policy) of the BID algorithm. The controi element of BID has the following steps [Liv82]:

1. If $n_{i}>1$ go to step 2 . else terminate the algorithm. 
2. Wait $D / n_{i}{ }^{4}$ units of time. $D$ is a parameter of the algorithm. The value of $D$ depends on the properties of the communication system.

3. Broadcast a reservation message if no other node has broadcasted such a message during the time-out period" If another node has succeeded to broadcast a reservation message terminate the algorithm.

4. Wait for a reply message. The reply will be positive if the node that has broadcasted the idle message is still idle. The node will send a reply in any case.

5. If the reply is positive and $n_{i}>1$ transfer a task to the idle node, else terminate the algorithm.

\subsection{The Poll When Idle Algorithm (PID)}

This policy differs from STD and BID in both the information policy and control element. The information policy of STD uses polling instead of broadcasting the state of a particular node. The information policy is regarded as demand driven, since it collects the load index of a subset of nodes in a distributed system. When a node becomes idle, it polls a subset of the nodes. The sequences of the polling operation of the PID policy are as follows [Liv82]:

- The purpose of the state-dependent time-out period is to give nodes with greater load a better chance to transfer a task to the idle node

s If the transmission of the message is delayed due to collisions the same condition is tested before an attempt to retransmit the message is made. 
1. Randomly select a set of $R$ nodes $\left(a_{i}, \ldots, a_{r}\right)$ and set $J=1$. $R$ is a parameter of the algorithm.

2. Send a message to node $a_{j}$ and wait for a reply.

3. Receive the reply message. Node $a_{j}$ will either send back one of its waiting tasks, if there are any, or an 'empty queue' reply.

4. If the node is still idle and $j<R$, increment $j$ and go to step 2 else terminate the polling.

Note that $\mathrm{R}$ is also called a probe limit in the literature. The control element uses a distributed algorithm. When a node is polled, the control element at that node determines whether the node will transfer a task to the idle (polling) node in step 3. Again, the selection policy only allows non-preemptive job transfers.

\subsection{Sender-Initiated Policies}

in sender-initiated policies, load sharing activity is initiated at a sender node in which it has an extra job to transfer. A sender node is identified by the transfer policy of the sender-initiated policy using a threshold T. Eager et al. [Eag86] have proposed three sender-initiated policies. Each of the three policies has the same selection policy, transfer policy and information policy with the exception of the random sender-initiated policy; however the location policy differs among the three. All of these sender-initiated policies 
are based on the same threshold transfer policy, non-preemptive selection policy and demand-driven information policy.

Under a threshold transfer policy, when a job arrites at a node. the job is processed locally if the number of tasks already in service or waiting for service (the node queue length) is less than some threshold $T$; otherwise the task will be transferred to a receiver node if there is one available. The receiver is determined by the location policy. Its selection policy permits only newly arrived jobs to be transferred. The information policy is a demand-driven policy in which a node only acquires other nodes' state information when it is necessary. There is no information policy in the random senderinitiated policy since the random sender-initiated policy does not require load information of other nodes to make transfer decisions. The following sub-sections discuss each type of sender-initiated load sharing policies in terms of the location policy in detail.

\subsubsection{Random Sender-Initiated Policy (RSI)}

In the random sender-initiated policy, the random location policy does not require any load information from any node in the system. The job which makes a node become a sender node is transferred to a destination node chosen at random. Under this policy, a receiving node should treat remote jobs just as jobs originating at itself. That is, if the receiver's queue length is less than $T$, then it will accept and process the task locally; otherwise the task will be transferred again to some other node selected at random. As 
mentioned in Eager et al.'s paper, without a suitable control policy, the job transfer process will definitely cause system instability regardless of system load. Thus, this policy includes a static transfer limit $L_{t}$. The destination node of the $L_{t}^{\text {th }}$ transfer of a job must execute the job even its current queue length is not less than threshold $T$.

\subsubsection{Threshold Sender-Initiated Policy (SI)}

This policy acquires load information of other nodes via the demand-driven information policy and uses this information to make decisions to which node a job should be transferred. When a node becomes a sender, it probes another node selected on a random basis. The threshold location policy initiated at a sender node checks whether a transfer of a job to the probed node would increase the queue length of the probed node above threshoid $T$. If this is not the case, the job will be transferred from that sender node to the probed node. The destination node must prociess the task regardless of its queue length when a task is actually transferred. If transferring a job from that sender node to the probed node would increase the node queue length above $T$, then another node is selected at random and probed in the same way. The process continues until either a suitable node is found or the sender has probed the probe limit $L_{p}$ times. In the latter case, the overloaded node must process the job locally. As demonstrated in Eager et al.'s study, the performance of the policy is not sensitive to the probe limit and a probe limit of 3 or 5 is almost as good as the performance with a large probe limit, e.g., 20 . 


\subsubsection{Shortest Sender-Initiated Policy (SSI)}

Under the shortest sender-initiated load sharing policy, the shortest location policy acquires more system state information and strives to make the best receiver node selection, given the probed load information of a subset of nodes. When a node is determined as a sender node by the transfer policy, the location policy selects $L_{p}$ distinct nodes at random and probes them, in turn, to determine their queue lengths. The job in a sender node is transferred to a node with the shortest queue length, unless that queue length is greater than or equal to the threshold, in which case the sender node must process the job locally. Similar to threshold policy, the receiving node must process the job regardless of its state. The authors have also described an improved version of shortest sender-initiated policy. In this case, when a node with an empty queue is probed then that node is considered as a transfer partner and the probing process is stopped, since it is guaranteed that the node is an acceptable destination node.

\subsection{Receiver-Initiated policy (RI)}

Receiver-initiated policy was studied by Eager et al. [Eag86]. This policy is like the "poll when idle" policy [Liv82] but the policy examined by Eager et al. is a generalized one. Thus, it is considered as a different type of policy for the sake of description. It is called a receiver-initiated policy because the load sharing is initiated by receiver nodes. A lightly loaded node (receiver) attempts to acquire a task from a heavily 
loaded node (sender) in order to reduce the load imbalance and improve the performance of the system. The information policy is demand-driven, as probing is required only when a job departs from a node which becomes a receiver node. The selection policy is preemptive under this same study. However, the selection policy can be non-preemptive. The performance of the receiver-initiated policy with a non-preemptive transfer has been studied in [Dan95a] and [Ben94]. The transfer policy and location policy work as follows. When a job departs from a node, the transfer policy determines whether the node is a receiver ( $A$ node is a receiver if its queue length is less than threshold $T$ ). If so, the location policy is invoked to look for a suitable sender. The information policy is invoked by the location policy to acquire necessary load information for choosing a suitable transfer partner. A node is selected at random and probed to obtain the queue length of the probed node. If the queue length of the probed node is above threshold $T$, that is a transfer of a job from that node would not reduce its queue length below the threshold $\mathrm{T}$, and that node is not in a process of transferring a task to another node, then a job is transferred to the node which initiating the probe. Otherwise another node is selected at random and probed again in the same manner. The process continues until the probing node exhausts the probe limit $L_{p}$ or a sender node is located. In the former case, the original node must wait until another task departs before attempting to transfer a job. When the threshold T is set to one, it is identical to Livny's "poll when idle" policy. 


\subsection{Reservation Policy (RES)}

The reservation policy was also studied by Eager et al.[Eag86]. The major goal of this policy is to eliminate the cost of preemptive transfers. That is. the selection policy is non-preemptive. In a multi-tasking system, the local node scheduling policy used is usually "Round-Robin". Under such an environment, where receiver-initiated policy is used, almost all job transfers involve partly-executed jobs. This is more costly than nonpreemptive job transfers, since when transferring a partly executed job, the state of the job must be transferred from a source node to a destination node. In RES a receiver node attempts to acquire a job from a sender node. When a job departs from a node, the distributed threshold transfer policy determines whether the queue length of a node is less than threshold $T$. If this is so it dispatches the demand-driven information policy which chooses a node at random and probes for load information. The distributed threshold location policy then decides if the transfer of the next job from the probed node would place its queue length below threshold T. If not, and there is no other pending reservation for the probed node, then the next job is reserved by the probing node. $\mathbf{A}$ job is transferred if no other jobs have arrived at the probing node when the reserved job arrives. When the probe fails, another node is selected at random and probed in the same manner. This process is repeated until either it has successfully reserved a job or a static poll limit number of trials has failed to locate a sender node. In the second case, the node must wait for the next departure before it can attempt to reserve a job again. 


\subsection{Symmetrically-Initiated Policy (STM)}

Symmetrically-initiated policy has been studied by Mirchandaney et al.[Mir89], Shivaratri et al. [Shi90], Krueger et al. [Kru88] and Benmohammed-Mahieddine et al. [Ben 94]. Under this policy, load sharing is initiated by both senders and receivers. A sender node attempts to transfer a job to a receiver node when the arrival of a job places its queue length above a static threshold $T$. On the other hand, a receiver attempts to acquire a job from a sender when the departure of a job cause its queue length to fall below a static threshold $\mathrm{T}$. The goal of this policy is to combine the advantages of both sender-initiated policy and receiver-initiated policy. It has been shown [Eag86] that at low to moderate system loads the threshold sender initiated policy outperforms the receiver-initiated policy. However at high to very high system loads (above $70 \%$ ) the receiver-initiated policy gives better performance when the costs of transfer is comparable to that of sender-initiated policy.

This policy is constructed by combining the transfer and location policies of threshold sender-initiated and receiver-initiated policies. That is, it has a sender-initiated component and a receiver-initiated component which are activated according to the current load of a node. The selection policy can involve both preemptive and nonpreemptive transfers. The symmetrically-initiated policy studied by BenmohammedMahieddine only allows non-preemptive transfers. The information policy is demand driven, as polling of other nodes' load information is only done when a node become 
either a sender or receiver. A node is identified as a sender by transfer policy if its queve length is above threshold $T$ when a new job arrives at the node, and identified as receiver if its queue length is below threshold $T$ when a job departs from the node. The probing procedure of the sender-initiated component and the receiver-initiated component is the same as that described in Section 2.5 .2 and 2.6 respectively.

\subsection{Stable Symmetrically Initiated Adaptive Policy (SSYM)}

The stable symmetrically-initiated adaptive policy was proposed by Shivaratri [Shi90]. It includes both a sender-initiated component and a receiver-initiated component and utilizes three state lists to store the system state. This policy combines the merits of sender-initiated and receiver-initiated policies while eliminating the unnecessary probing generated by the sender-initiated component at high system loads, in order to maintain system stability at high system loads. It has been mentioned in the previous section that the receiver-initiated policy performs well at high loads while sender-initiated policy excels at low to moderate loads. Its transfer policy classifies nodes into three states: sender, OK and receiver. A node is a sender if its queue length is greater than some threshold T. A node is an OK if its queue length is equal to $T$. A node is a receiver if its queue length is less than $T$.

An efficient data structure is used to maintain the three state lists at each node $i$ : a sender list $\left(\right.$ Slist $\left.{ }_{i}\right)$, a receiver list (Rlist $\left.t_{i}\right)$ and an OK list $\left(\mathrm{OKlist}_{\mathrm{i}}\right)$. All nodes fall into one 
of the three lists at each node. The SSYM consists of two negotiations: the senderinitiated negotiation and the receiver-initiated negotiation. During a sender-initiated negotiation the sender node seeks a receiver node. In contrast, during a receivernegotiation a receiver node seeks a sender node. The policy works as follows [Shi90]:

\section{Initialization:}

Initially all sodes assume that every other node is idle, and therefore a potential receiver. For node $\mathrm{i}$ in a distributed system containing $\mathbf{n}$ nodes:

Rlist $_{i}=i+1, i+2, \ldots, n, 1, \ldots, i-1$ OKlist $t_{j}=$ null; Slist $t_{i}=$ null. This ordering for Rlists is not required for correctness, but it helps in initially dispersing negotiation activity among the nodes.

Sender-Initiated Negotiation:

During sender-initiated negotiation, an attempt is made at sender to find a receiver. To do so, the location policy at sender node $i$ does the following:

1. Probe the node at the head of Rlisti , say $j$, to determine whether $j$ is receiver.

2. If $\mathbf{j}$ identifies itself as receiver, return its ID to transfer policy and Stop. If $j$ is not a receiver, remove it form Rlisti and add it to the head of either Slist ${ }_{i}$ if $j$ is a sender, or to OKlist $_{\mathrm{i}}$ if $\mathrm{j}$ is $\mathrm{OK}$. 
3. STOP negotiation and return FAILURE to the transfer policy if either $i$ has probed probelimit nodes (where probelimit is a parameter of the algorithm) without success. if Rlist $t_{\mathrm{i}}$ is empty, or if $\mathrm{i}$ is no longer a potential sender. Otherwise go to step 1.

At probed node j:

1. On receipt of a probe message from node $i$, remove $i$ from whatever list it is in and add it to the head of Slist $t_{j}$ (Note: If $i$ was already in Slist $t_{j}$ this action simply changes its position in the list).

2. Send a reply message to $i$ indicating whether $j$ is a receiver sender or OK(determined by the transfer at node $\mathbf{j}$ ).

Receiver-Initiated Negotiation:

During receiver-initiated negotiation, an attempt is made by the receiver to find a sender. The location policy at a receiver node $i$ does the following:

1. Probe a selected node, say $j$, to determine whether $j$ is a sender. The node selected for probing is chosen as follows: If possible, choose the node at the head of Slist (most up-to-date information is used first). If Slist $t_{i}$ is empty, choose the last node from Oklist $t_{i}$ (most out of date information is used in the hope that the state has changed from OK to sender). If both OKlist ${ }_{i}$ and Slist $t_{i}$ are empty, choose the last node from Rlist $t_{i}$ (again the most out-of date information is used first). Note that the 
contents of the lists may change as negotiation proceeds, because of other negotiations occurring in parallel with this negotiation. Nodes that join Slist ${ }_{i}$ in this way may be probed in the current session. Nodes joining either OKlist $t_{i}$ or Rlist in this way, however, are not considered for probing, since it is unlikely that they would have become suitable senders in such a short time.

2. If $\mathrm{j}$ responds that it is a sender and that it will remain a sender even after migrating a task, add it to the head of Slist, return its ID to the transfer policy, and STOP. If $j$ responds that it is a sender but will no longer be a sender after migrating a task, remove it from whatever list it is in and add it to the head of OKlist, return its ID to the transfer policy, and STOP. If $\mathrm{j}$ responds that it is not a sender, remove it from whatever list it is in and add it to the head of either Rlist if it is a receiver, or OKlist if it is OK.

3. Stop negotiation and return FAILURE to the transfer policy if either: $i$ is no longer a receiver, $\mathrm{i}$ has probed probelimit nodes without success, or all the nodes that might be considered for probing (see step 1) have been probed. Otherwise go to step 1.

At probed node $j$ :

1. If $j$ is not a sender, return a message informing $i$ of its state (receiver or OK). Remove $i$ from whatever list it is in and add it to the head of Rlist $t_{j}$ 
2. If $j$ is a sender, migrate a lask to $i$. Inform $i$ of $j$ 's state after the migration.

The sender-initiated component only considers newly arrived jobs. Conversely, the receiver-initiated component considers partly-executed jobs as well as newly arrived jobs. Thus the selection policy of SSYM is both preemptive. The information policy of SSYM is demand-driven since load information is collected only when a node becomes either a sender or receiver.

\subsection{Stable Sender-Initiated Adaptive Policy (SSIA)}

This policy was also proposed by Shivaratri et al. [Shi90] and uses the same sender-initiated components, but different receiver-initiated components which are directed at reducing the cost of preemptive transfers by transferring newly arrived jobs only. This policy uses a state vector of size $n$ where $n$ is the system size, in addition to the three state lists. The state vector keeps track of what state a node is perceived by other nodes. That is, $S V_{i}[j]$ gives which list node $i$ belongs at the node $j . T$ The $S V_{i}[j]$ is defined as:

$S V_{i}[j]=0$ if $i$ knows that $i \in R$ list;

$S V_{i}[j]=1$ if $i$ knows that $i \in$ OKlist $_{j}$

$S V_{i}[j]=2$ if $i$ knows that $i \in$ Slist $_{j}$ 
The state vectors are initialized as: $\forall \mathrm{i}, \mathrm{j} i \neq \mathrm{j} S V_{\mathrm{i}}[\mathrm{i}]=0$ i.e. all nodes know that they are identified as receivers by other nodes initially. During a sender-initiated negotiation at probing node $\mathrm{i}$, this policy follows the same steps as that in Section 2.8 but after step 1 , $S V_{i j}[j]$ is set to a value of 2 which means that $i$ is in Slist $t_{j}$. At probed node $j$ : All the steps of Section 2.8 are carried out with the state vector set according to its reply below:

$S V_{j}[i]=0$ if $j$ replied 'receiver', i.e. $j \in R$ Rlist $_{i}$;

$S V_{j}[i]=0$ if $j$ replied 'OK', i.e. $j \in$ OKlist $_{\mathrm{i}}$;

$S V_{j}[i]=2$ if $j$ replied 'sender' $i . e . j \in$ Slist $_{\mathrm{i}}$.

During receiver-initiated negotiation, at receiver node i [Shi90]:

$\forall j . i \neq j$ do, it $S V i[j] \neq 0$ (i.e. , $i \notin R$ Rlist $t_{i}$, hence $j$ will not probe $i$ even $j$ is a sender) then inform $j$ that $i$ is a potential receiver and set $S V_{i}[j]$ to zero .

At informed node $j$ :

On receipt of a state-change message from $i$, remove $i$ from whatever list it is in, and add $i$ to the head of Rlist ${ }_{i}$ Note: If $i$ was already present in Rlist $t_{j}$, this action simply changes its position in the list). 


\subsection{Single Coordinator Policy}

There are many variations of a single coordinate policy but the main ideas of the single coordinate policy is to centralize load sharing decisions. This section discusses a receiver-initiated single coordinate policy [Dan96] in which a receiver node attempts to acquire a job from a sender node.

Each node in a distributed system can be in one of three states: SENDER, OK and RECEIVER. When a node changes its state due to an arrival or a departure of a job, it sends a state change message to the coordinator, called the supervisor node. indicating whether the nodes are in SENDER, OK or RECEIVER state, i.e., the information policy is state driven. Unlike stable symmetrically-initiated adaptive policy, the SINCO transfer policy determines the state of a node according to two threshold values, $T_{1}$ (lower threshold) and $T_{h}$ (upper threshold) where $T_{h} \geq T_{1}$. If its queue length is above threshold $T_{h}$, then it is a sender. If its queue length is below threshold $T_{1}$, then it is a receiver. Otherwise the node is in an OK state.

When a node becomes a receiver, then it will inform the supervisor node it needs a job. The supervisor will search its state array, $S V=\left(S_{1}, \ldots, S_{n-1}\right)$, which consists of the states (SENDER, RECEIVER, OK) of all the nodes in the system. If there is one or more sender nodes, then a sender is chosen at random. If a sender node is found, then the supervisor node sends a job request message to the sender on behalf of the receiver. If 
the supervisor cannot locate a suitable sender, then it will retum a 'FAILURE' reply to the receiver node. When a receiver node receives such a message, it sends a state change message to the supervisor to indicate it is now a receiver node. When a receiver node receives such messages, it also has to wait for a re-initiation period before it can consult the supervisor again (provided that it is still a receiver). The re-initiation of a load distribution is necessary in order to improve its performance under certain system and workload conditions.

\subsection{Periodic Symmetrically-Initiated Policy (PSI)}

PSI was proposed by Benmohammed-Mahieddine at el. [Ben94]. This policy consists of two major components: the sender-initiated and the receiver-initiated components. The transfer policy is triggered by a timer associated with each node that, in turn, dispatches the RI or SI components depending on the current load of a node. The probe limit $L_{p}$ of each component is set to one. That is, a node can, at most, probe one node for each initiation of either an SI or RI component. The goal of the policy is to reduce the overhead incurred during probing. The selection policy of PSI is nonpreemptive as only newly arrived jobs are eligible for transfer. At each timer period of a node, the transfer policy checks the load index of the node against its threshold $T$ and does one of the following [Ben94]: 
if exceeding the threshold (load.i $>T+1$ ), a request is sent to a random node $\left(L_{p}=1\right)$, the node replies with an ACCEPT message if it is underloaded (load.j $<T+1$ ), otherwise it ignores the request. The requesting node transfers a job from its transferable jobs queue as a response to an ACCEPT message (pushed job), or ignores the request if it is no longer overloaded.

if below the threshold (load. $i<T+1)$, a request to receive a job is made to a random node. The chosen node will respond by sending a job from its transferable jobs queue (pulled job), or just ignores the message if it is also underloaded (load.j $<T+1$ ).

if the load is normal (load. $\mathrm{i}=\mathbf{T}+1$ ), no load balancing is attempted.

\subsection{Hierarchical Receiver-Initiated Policy (HRI)}

This policy was proposed by Dandamudi and Lo in [Dan96]. This policy uses a tree-like structure instead of a single node to store the state of nodes in order to make load sharing decisions. It captures the essence of the "single coordinator" policy described in Section 2.10. It will be shown later in Chapter 3 that the "single coordinator" policy is relatively insensitive to variance in job service times and inter-arrival times, and yields the best performance among the other policies compared. However, the single coordinator may cause fault-tolerance and scalability problems. In this context, it should be noted that some studies have shown that a single node collecting and maintaining the

system state need not be a performance bottleneck for reasonably large distributed 
systems [The88]. However, if a system is geographically distributed (for example, several LAN clusters interconnected by a WAN), consulting a central node is very expensive and causes performance problems. Thus, the use of the centralized single ccordinator policy is often limited to a cluster of nodes in a large distributed system.

The hierarchical policy strives to eliminate the disadvantages of the centralized single coordinator policy mentioned above, while providing performance very close to that of the single coordinator policy and fitting the inherent structure of some large distributed systems where several LAN clusters are interconnected by a WAN.

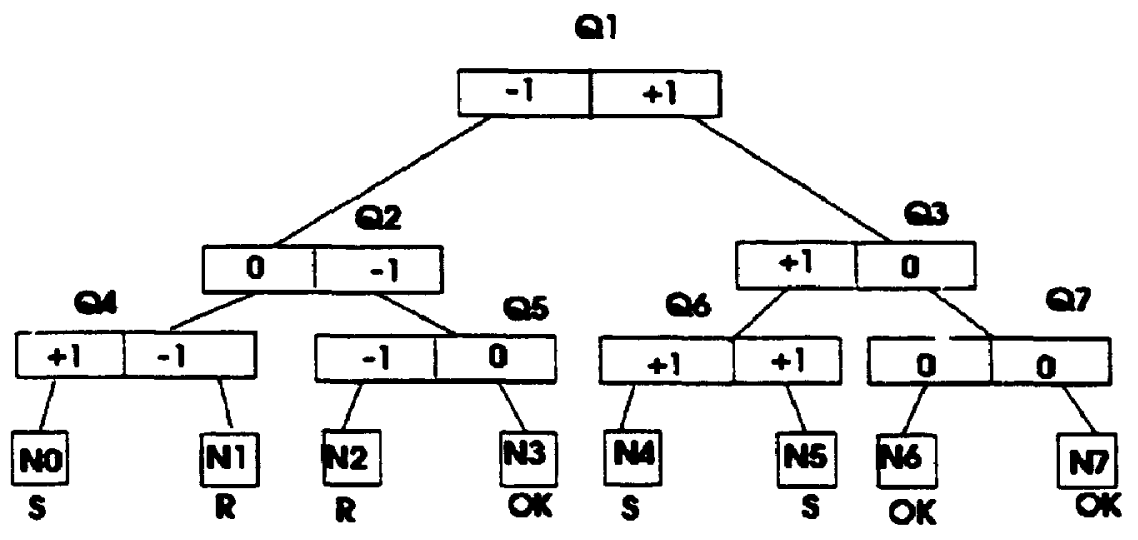

Figure 2.1 An example of a local hierarchical organization for an 8-node system with a branching of 2.

In the hierarchical policy, instead of a single node maintaining the entire system state, a set of nodes is given this responsibility. The system is logically divided into clusters and each cluster of nodes will have a single node that maintains the state information of the nodes within the cluster. The state information on the whole system is maintained in the form of a tree where each tree node maintains the state information on 
the set of processor nodes in the sub-tree rooted by the tree node. Figure 2.1 shows an example hierarchical organization for $\mathbf{8}$ processor nodes with a branching factor $\mathbf{B}$ of 2 . Recall that a node can be in one of three states: sender (overloaded), OK (normal load), or receiver (underloaded). We will use +1 to represent the sender state, 0 for the $\mathrm{OK}$ state, and -1 for the receiver state. For example, $Q 4$ maintains the state information in nodes NO ands N1. It may be noted from Figure 2.1 that each node in the tree maintains the state information on all the nodes in the sub-tree rooted at this tree node. In other words, cluster size increases as we move up the tree. For example, Q2 maintains state information on nodes $\mathrm{N} 0, \mathrm{~N} 1, \mathrm{~N} 2$, and $\mathrm{N} 3$. towever, in order to reduce the amount of information that has to be kept at higher tree nodes, only summary state information is maintained. The summary metric used in this policy is a interpretation of arithmetic sum of the state information of the tree nodes below it. For example, summary state metric for Q4 is zero, which implies that the cluster represented by Q4 (i.e., nodes N0 and N1) is in OK state. Thus this policy encourages local load sharing . For this reason, this policy is called the local hierarchical policy. For example, the summary metric for Q6 stored in Q3 is +1 rather than +2 . Note that the value stored in the parent node is +1 if the sum is positive, -1 if it is negative and 0 otherwise. An advantage of this scheme is that it reduces the number of ipdates required to maintain the hierarchy. For example, if the system state changes and N4 moves to OK state, only the entry in Q6 needs to be changed. Since N5 is still a sender and the cluster represented by Q6 is still in SENDER 
state $(+1)$, no state change is necessary for Q3. In addition, two global hierarchical policies are discussed in Chapter 3.

Since load sharing can be done at various levels of the tree, the root node does not have to handle requests form all nodes in the system. Furthermore, it can also be seen that the set of nodes that form the tree can all be distributed to different system nodes so that no node is unduly overloaded with system state information. For a system with $\mathbf{N}$ nodes, the maximum number $(\mathrm{N}-1)$ of tree nodes will be required when the branching factor $B$ is 2 . Since there are $N$ system nodes, these $(\mathrm{N}-1)$ tree nodes can be distributed uniformly.

The choice of the tree branching factor is a key design issue in such an organization. We wish to keep the tree as "bushy" as possible (i.e., a tree with a large branching factor) because it allows better load sharing and results in better performance. Taken to the extreme this represents the centralized single coordinator organization. In Chapter 3, the impact of the branching factor on the performance of the hierarchical policies will be studied. With this hierarchical structure in place, various load sharing policies can be implemented in conjunction with it. Here we describe a receiver-initiated policy that works with this hierarchy. Similar to the basic RI, its load sharing is activated when a node is identified by the transfer policy as a receiver. The receiver then consults 
the hierarchy (explained next) to locate a sender. The local hierarchical policy works in the following manner.

When a job is completed at a node, if there are no jobs in its local job queue, it sends a message to its parent tree queue (i.e., to the processor node that is responsible for maintaining this tree node) requesting a sender node. If the tree node does not have any sender nodes in its sphere, it forwards the message to its parent tree node. This process is repeated up the tree until either a tree node with a sender node is encountered or the root node is reached. If the root node does not have a sender node, implying that there is no sender node in the whole system, a "no job" message is sent back to the receiver node that initiated the request. If a tree node with at least one sender branch is found, the message follows a "downward" path along this branch until it reaches the sender node. If the tree node has more than one sender branch, one is selected at random. When the message reaches the sender node, if that node is still a sender, it transfers a job to the receiver node. If, on the other hand, the node is no longer a sender (as it is in the process of updating its entry in the hierarchy), it sends a "false sender" message to the receiver indicating that there is no job to transfer. When a "no job" or a "false sender" message is received by the receiver node, and if the node is still in the receiver state (i.e., there were no local job arrivals since the request), it updates its entry in the hierarchy (to receiver state), waits for the corresponding re-initiation period and initiates another load 
distribution request (if it remains in the receiver state at the end of the re-initiation period).

\subsection{Goals and Motivation}

In this chapter, a survey of load sharing policies is presented. Many of these load sharing policies have not been compared under the same workload and system models. Moreover, many performance studies have assumed that the job inter-arrival times and the job service times be exponentially distributed, and that the distributed system be homogeneous. Thus, the goal of this thesis is to select some representative load sharing policies for performance evaluation under the same workload and system models and under both homogeneous and heterogeneous systems. First, six load sharing policies are chosen for the study of their performance in a homogeneous system. These policies are the sender-initiated, receiver-initiated, hierarchical receiver-initiated, periodic symmetrically-initiated, single coordinator and stable symmetrically-initiated adaptive policies. The sender-initiated and receiver-initiated policies are chosen for the completeness of this study. The periodic symmetrically-initiated and the stable symmetrically-initiated adaptive policies are selected because it has been demonstrated in [Ben94] that the periodic symmetrically-initiated policy excels sender-initiated and recsiver-initiated policies and in [Shi90] that stable symmetrically-initiated policy provides superior performance over sender-initiated and receiver-initiated policies. However both periodic symmetrically-initiated and stable symmetrically-initiated 
adaptive policies have not been compared under the same system and workload models. Although single coordinator and hierarchical receiver-initiated policies have been studied in [Dan96], they have not been evaluated with these two distributed policies. Only five of the six policies are selected for the study of their performance in two types of heterogeneous systems. The performance of the periodic symmetrically-initiated policy in heterogeneous systems is not studied because it does not perform as well as the stable symmetrically-initiated adaptive policy at high system loads in homogeneous systems. even though both are symmetrically initiated load sharing policies. Two variations of the local hierarchical policy are aiso discussed, and their performance in both homogeneous and heterogeneous systems is evaluated in Chapter 3 and 4 respectively. 


\section{CHAPTER III \\ PERFORMANCE OF LOAD SHARING POLICIES IN A HOMOGENEOUS SYSTEM}

\subsection{Introduction}

This chapter presents the results of the simulation experiments on the six selected load sharing policies introduced in Chapter 2. Section 3.2 describes the system and workload models. The transfer and selection policies of sender-initiated, receiver-initiated, periodic symmetrically-initiated and stable symmetrically-initiated adaptive policies are modified as described in Section 3.3 in order to allow non-preemptive job transfers only, and to fit into the model described in Section 3.2. Section 3.4 evaluates the performance of the load sharing policies on different system and workload parameters and on the parameters of those policies. Section 3.5 introduces two variations of the local hierarchical policy: namely the global non-recursive, and global recursive hieranchical receiver-initiated policies. The performance of these hierarchical policies is reported in Section 3.6. In concluding this chapter a summary of findings of these experiments is presented.

\subsection{System and Workload Models}

For this study the system model and workload model are the same models used in [Dan96a. Dan96b]. In this system model (see Figure 3.1), a locally distributed system is represented by a collection of nodes. In this chapter, the nodes are assumed to be homogeneous. In other words, they are subjected to the same mean inter-arrival time and mean job service demand. Each node maintains two job queues, a job-wait queue and a job 
queue. The job wait-queue stores jobs that are eligible for remote execution and local execution and the job queue stores jobs that must be executed locally such that preemptive job transfers can be avoided. The communication network in the system is modeled at a higher level. Communication delays are modeled without modeling the low-level protocol details. An Ethernet-like network with $10 \mathrm{Mbits} / \mathrm{sec}$ is assumed. The communication network is modeled as a single server. Each node is assumed to have a communication processor (CP) that is responsible for handling communication with other nodes. Similar assumptions have been made by other researchers [Mir89]. The CPU would give preemptive priority to communication activities (such as reading a message received by the communication processor, initiating the communication processor to send a probe message. etc.) over the processing of jobs.

The CPU overheads that are involved in sending/receiving a probe and a standard message and in transferring a job, are modeled by $T_{\text {probe, }} T_{m s g}$ and $T_{j x}$, respectively. A standard message is a message for updating the state of a node, sending job request, sending a search message to the cluster coordinator or replying requests (except transferring a job which represents a reply in some policies). Note that not all CPU overheads are applicable to all load sharing policies (see Table 3.1). For example, in hierarchical policy (LHRI) node does not need to probe node(s) for load information but needs to notify its coordinator about its state which is done through a standard message. Actual job transmission (handled by the communication processor) time is assumed to be uniformly distributed between $U_{j x}$ and $L_{j x}$. Probe messages as well as state information messages are assumed to be transmitted 
serially, not in parallel. For example, the implementation in [Dik89] uses serial probing. Messages and probes are processed in a "First In First Out" order.

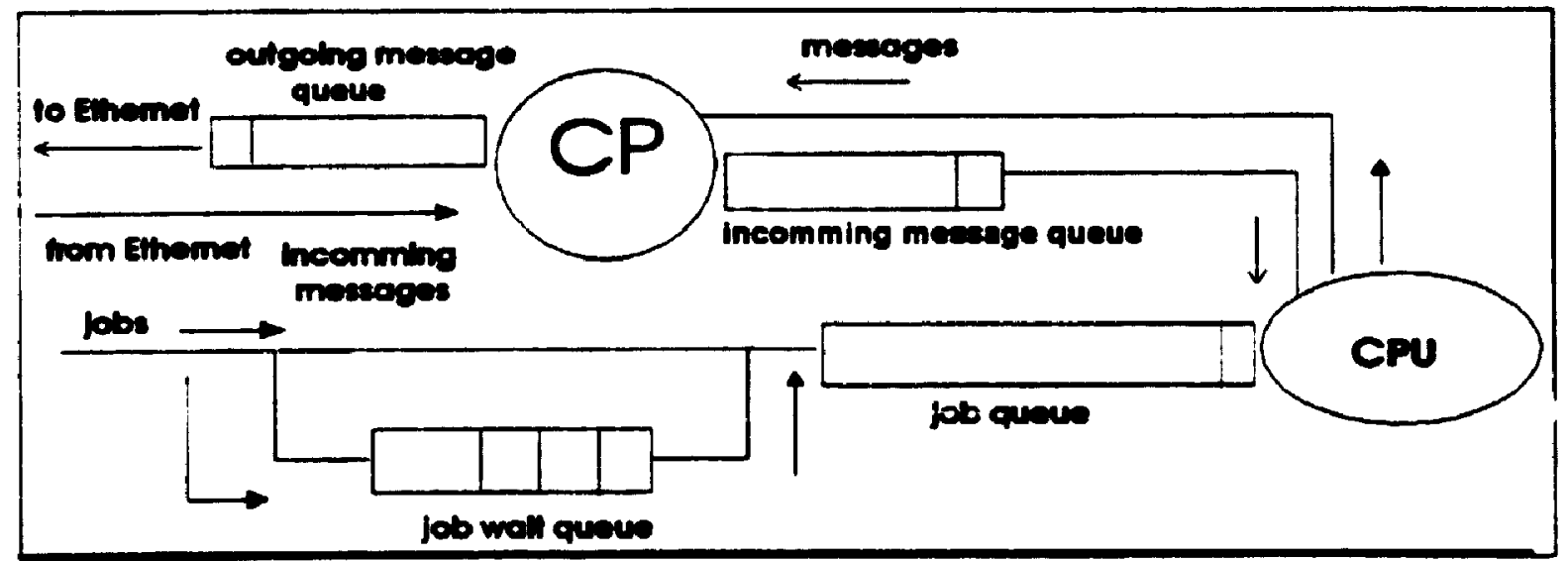

\section{A model of a single node}

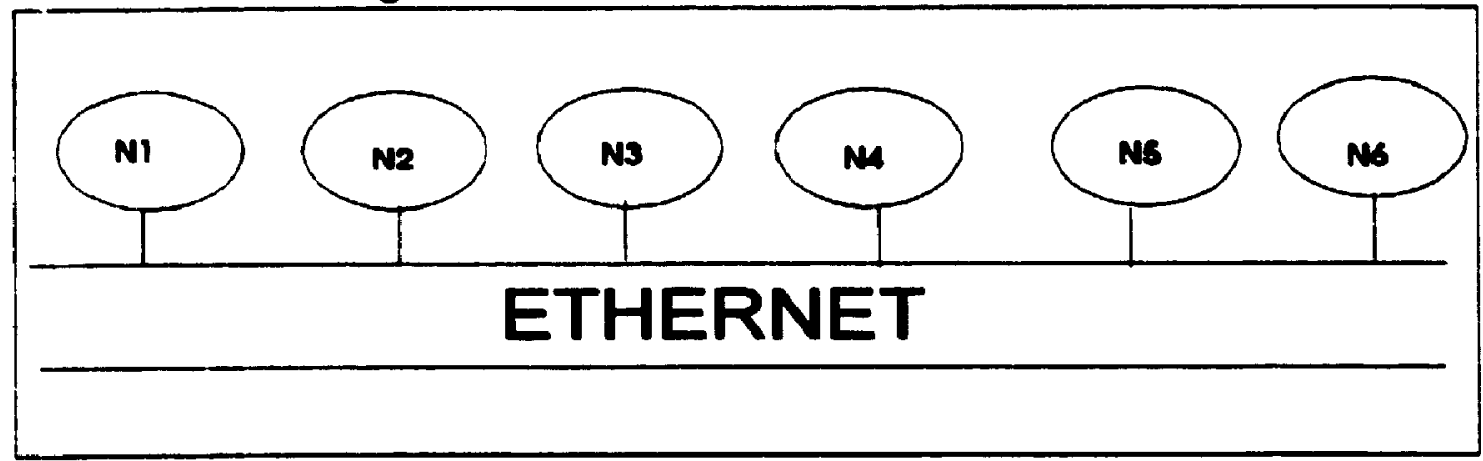

A model of distributed system with 6 nodes

Figure 3.1 A system model of a typical distributed computer system

The system workload is represented by four parameters. The job arrival process at each node is characterized by a mean inter-arrival time $1 / \lambda$ and a coefficient of variation $C_{2}$. Jobs are characterized by a processor service demand (with mean $1 / \mu$ ) and a coefficient of variation $\mathrm{C}_{5}$. The performance sensitivity to variance in both inter-arrival times and service times is studied in Section 3.4.2. and 3.4.3 respectively (the coefficient of variations $C_{a}$ and $C_{s}$ are varied from 0 to 4). A two-stage hyper-exponential model has been used to generate service time and inter-arrival time CVs greater than 1 [Kob81]. As in most previous 
studies, only CPU-intensive jobs are modeled in this thesis. The mean response time is used as the main performance metric to compare the performance of the various load sharing policies.

\begin{tabular}{|c|c|c|c|c|c|c|c|}
\hline Parmeter & Description & SI & $\mathbf{R I}$ & PSI & LHRI & SSYM & SINC() \\
\hline$\lambda$ & Mean job arival rate & $\mathbf{Y}$ & $\mathbf{Y}$ & $\mathbf{Y}$ & $\mathbf{Y}$ & $\mathbf{Y}$ & $\mathbf{Y}$ \\
\hline $\boldsymbol{\mu}$ & Mean job service rate & $\mathbf{Y}$ & $\mathbf{Y}$ & $\mathbf{Y}$ & $\mathbf{Y}$ & $\mathbf{Y}$ & $\mathbf{Y}$ \\
\hline $\mathbf{C}_{\mathbf{a}}$ & Inter-arrival time coefficient of variations & $\mathbf{Y}$ & $\mathbf{Y}$ & $\mathbf{Y}$ & $\mathbf{Y}$ & $\mathbf{Y}$ & $\mathbf{Y}$ \\
\hline $\mathrm{C}_{\mathrm{s}}$ & Service demand coefincient of variations & $\overline{\mathbf{Y}}$ & $\bar{Y}$ & $\overline{\mathbf{Y}}$ & $\overline{\mathbf{Y}}$ & $\mathbf{Y}$ & $\mathbf{Y}$ \\
\hline $\mathbf{U}_{\mathrm{jx}}$ & Upper job trensmission time limit & $\mathbf{Y}$ & $\mathbf{Y}$ & $\mathbf{Y}$ & $\mathbf{Y}$ & $\mathbf{Y}$ & $\mathbf{Y}$ \\
\hline $\mathbf{L}_{\mathbf{j k}}$ & Lower job transmission time limit & $\mathbf{Y}$ & $\mathbf{Y}$ & $\mathbf{Y}$ & $\mathbf{Y}$ & $\mathbf{Y}$ & $\mathbf{Y}$ \\
\hline $\mathbf{T}_{\mathbf{m}}$ & Threshold for transfer policy & $\mathbf{Y}$ & $\mathbf{Y}$ & $\mathbf{N}$ & $\mathbf{N}$ & $\mathbf{N}$ & $\mathbf{N}$ \\
\hline T, & Sender thresh:old & $\mathbf{Y}$ & $\mathbf{Y}$ & $\mathbf{N}$ & $\mathbf{N}$ & $\mathbf{N}$ & $\mathbf{N}$ \\
\hline $\mathrm{T}_{1} / \mathrm{T}_{\mathrm{h}}$ & Lower and upper threshold & $\mathbf{N}$ & $\mathbf{N}$ & $\mathbf{N}$ & $\mathbf{Y}$ & $\mathbf{N}$ & $\mathbf{Y}$ \\
\hline $\mathbf{T}$ & Threshold classifies senders and receivers & $\mathbf{N}$ & $\mathbf{N}$ & $\mathbf{Y}$ & $\mathbf{N}$ & $\bar{Y}$ & $\mathbf{N}$ \\
\hline$T_{\operatorname{man}}$ & CPU standard message overhead & $\mathbf{Y}$ & $\mathbf{Y}$ & $\mathbf{Y}$ & $\mathbf{Y}$ & $\mathbf{Y}$ & $\mathbf{Y}$ \\
\hline$T_{\text {prowe }}$ & CPU Probing message overhead & $\mathbf{Y}$ & $\mathbf{Y}$ & $\mathbf{Y}$ & $\mathbf{N}$ & $\mathbf{Y}$ & $\mathbf{N}$ \\
\hline$\overline{T_{\text {wat }}}$ & Re-initiation period for '10 partner' & $\mathbf{N}$ & $\bar{Y}$ & $\mathbf{N}$ & $\mathbf{Y}$ & $\mathbf{N}$ & $\mathrm{Y}$ \\
\hline$T_{\text {fine }}$ & Re-initiation period for 'false sender' & $\mathbf{N}$ & $\mathbf{N}$ & $\mathbf{N}$ & $\mathbf{Y}$ & $\mathbf{N}$ & $\bar{Y}$ \\
\hline $\mathbf{T}_{\mathrm{jx}}$ & CPU job trensfer overhend & $\bar{Y}$ & $\bar{Y}$ & $\bar{Y}$ & $\bar{Y}$ & $\mathbf{Y}$ & $\mathbf{Y}$ \\
\hline$T_{\text {pares }}$ & Timer period for initiation of location policy & $\bar{N}$ & $\mathbf{N}$ & $\mathbf{Y}$ & $\mathbf{N}$ & $\mathbf{N}$ & $\bar{N}$ \\
\hline$\overline{\mathbf{B}}$ & Branching factor & $\bar{N}$ & $\mathbf{N}$ & $\mathbf{N}$ & $\overline{\mathbf{Y}}$ & $\mathbf{N}$ & $\mathbf{N}$ \\
\hline $\mathbf{P}_{1}$ & Probe limit & $\mathbf{Y}$ & $\mathbf{Y}$ & $\mathbf{Y}$ & $\mathbf{N}$ & $\mathbf{Y}$ & $\mathbf{N}$ \\
\hline
\end{tabular}

Table 3.1 Input parameters

Table 3.1 summarizes all the input parameters, gives their descriptions and indicates their applicability. Some of the parameters are not applicable to all load sharing policies. The ' $\mathrm{Y}$ ' indicates that the parameter in the row is used by the policy in the intersecting colunin. For example, only PSI has a timer period for triggering the control elements (see 
Section 2.11), so only the cell on the same column of PSI has a ' $Y$ and all other cells have an ' $N$ '.

\subsection{Modifications to Load Sharing Policies}

This section describes the modifications made to the load sharing policies in order to support non-preemptive transfers and to fit the model described in Section 3.2. Most of the changes are made to the transfer policy, and the location policy which is the major component that affects the performance of a policy is not changed except that in the senderinitiated and receiver-initiated policies, their transfer and location policies use threshold $T_{m}$ and $T_{s}$ respectively instead of a single threshold as described in Chapter 2. The following paragraphs only describe the modifications.

In this study, the transfer policy and the selection policy of sender-initiated(SI), receiver-initiated (RI), periodic symmetrically-initiated(PSI) and stable symmetricallyinitiated adaptive policies needed to be modified to allow only non-preemptive transfers. For all load sharing policies, the selection policy only permits non-preemptive transfers. In order to avoid non-preemptive transfers, two queues are required. A job queue consists of all the jobs waiting to be executed as well as the job currently being executed. A job-wait queue contains those jobs that are eligible for a transfer (see Figure 3.1). The following are the modifications made to the load sharing policies:

Sender-initiated policy (SI): When a job arrives at a node, if the new job would place the job queue length above threshold $T_{m}$, then the transfer policy identifies the node as 
a sender and moves the job to the job-wait queue. The job is eligible for remote execution and transferred to a receiver node if there is one found through the probing process initiated by the location policy. A receiver node is a node at which its job queue is less than $T_{s}$. Note that if a receiver cannot be found, the sender node must process the job. The job will be transferred to the job queue from the job-wait queue.

Receiver-initiated policy (RI): The transfer policy of a receiver-initiated policy is the same policy as the transfer policy of a sender-initiated policy. When a job arrives at a node, if the job places job queue length of that node above $T_{m}$, then the job is placed into the wait queue. When a job departs from a node, and if the job-wait queue is not empty, a job is moved from the job-wait queue to the job queue. Otherwist, he location policy checks if its job queue length is less than threshold $T_{s}$. If so, the probing process is initiated to locate a sender node which has a non-empty job-wait queue.

Periodic symmetrically-initiated policy (PSI): The transfer policy is similar to that of the sender-initiated and receiver-initiated policies except that it does not trigger the location policy instead the location policy is triggered at each timer period $\left(\mathrm{T}_{\text {period }}\right)$. When a job arrives, the transfer policy must also determine in which queue a job should be put in order to allow non-preemptive transfers. When a job arrives, if the new job would place the job queue length above $T+1$, then the job is inserted into the job-wait queue; otherwise it is inserted into the job queue. When a job departs, the transfer policy moves a job from the job-wait queue to the job qur $\rightarrow$ if the job-wait queue is not empty. 
At each timer period, if a node is a sender (load.i $>T+1$ ) but the location policy fails to locate a receiver for that node, then the extra job remains at the job-wait queue and it is still eligible for remote execution iaitiated by a receiver or when another time period elapsed and the node is still a sender. The location policy of PSI is the same policy as that described in Section 2.11 .

Stable symmetrically-initiated adaptive policy (SSYM): The transfer policy of SSYM classifies the node into one of three states: sender, OK, and receiver. When a job arrives the policy inserts the job to either the job queue. if the node is in OK or receiver state, or to the job-wait queue if the node is a sender. The rest of the transfer policy and the location policy are not modified. (see Section 2.9).

Single coordinator and hierarchical policies: There is no change in the single coordinator policy except that when a job request is sent to a false sender where there is no extra job for remote execution, the request will be immeciately sent back to the single coordinator. The single coordinator will select a new potential sender for the initiator of that job request again. This process, which is like back tracking in the global recursive policy described later in Chapter 3, continues until either there is no more potential sender in the system or the request is satisfied. The local hierarchical receiver-initiated policy is not altered. 


\subsection{Performance Comparison}

The following are default parameter values assumed in the performance analysis in this chapter, unless otherwise specified. The distributed computing system consists of $\mathrm{N}=32$ nodes which are interconnected by a $10 \mathrm{megabit} / \mathrm{second}$ communication network. The average job service time is one time unit. The size of a probe message and a standard message is 16 bytes. The CPU overhead to send/receive probe $T_{\text {prube }}$ or send/receive standard message $T_{\text {msg }}$ is 0.003 and to job transfer $T_{j x}$ is 0.02 . The load distribution reinitiation period $T_{\text {wait }}\left(T_{\text {false }}\right)$ when a 'no-job'('false-sender') message is received is fixed at $1(0.2)$. The timer period $T_{\text {period }}$ for PSI is 0.4 . The thresholds $T_{1}$ and $T_{h}$ for LHRI and SINCO are both 1. The threshold T for PSI and SSYM is 0 and 1 respectively. The threshold $T_{s}$ is 2 and 1 for SI and RI respectively. The threshold $T_{m}$ is 2 for both $S I$ and RI. Note that although the values of threshold $T$ and $T_{s}$ are different, both values cause the load sharing policies to identify a node as a receiver node only if it is an idle node. These threshold values are used because a node is better off avoiding load sharing when there is work to do at a node.

The job transfer communication overhead is uniformly distributed between $\mathrm{L}_{\mathrm{jx}}=0.009$ and $\mathrm{U}_{\mathrm{jx}}=0.011$ time units (i.e., average job transfer communication overhead is $1 \%$ of the average job service time). Since only non-executing jobs are considered for transfer, $1 \%$ is a reasonable value. Note that transferring active jobs would incur substantial overhead, as the system state would also have to be transferred. A probe limit $P_{1}$ of 3 is used for the sender-initiated, the receiver-initiated and the stable symmetrically-initiated 
policies. For the hierarchical policy, the default branching factor is fixed at 4 . Section 3.5 discusses the impact of this parameter on the performance of the hierarchical policies.

Batch strategy has been used to compute confidence intervals. At least 30 batch runs with 30,000 jobs each were used for the results reported, except at very high CV values as well as high offered loads, and low offered loads. At high CV values and high offered loads, at least 50 batch runs with 50,000 jobs per run were used. At low system loads it is sufficient to use 10,000 jobs per nun; still 30 batches were used. This strategy has produced $95 \%$ confidence intervals, that were less than $1 \%$ of the mean response times when the system utilization is low to moderate, and less than $5 \%$ for moderate to high system utilization. At a very high load (90\% utilization) and both CVs $=4$, the $95 \%$ confidence intervals are about $10 \%$ of the job mean response time.

\subsubsection{Performance as a Function of System Load}

Figures 3.2 and 3.3 plot the job mean response time against offered system load.

The offered system load is given by $\lambda \mu$. Since $\mu=1$ for all experiments, offered system load is equal to $\lambda$. The results in Figure 3.2 correspond to inter-arrival coefficient of variation $\mathrm{C}_{\mathrm{a}}$ and service time coefficient of variation $\mathrm{C}_{s}$ of 1 . The results shown in Figure 3.3 is obtained with both $C_{s}$ and $C_{a}$ of 4 . The following are the main observations from Figure 3.2(a) which corresponds to the $C_{a}$ and $C_{s}$ of 1 . 


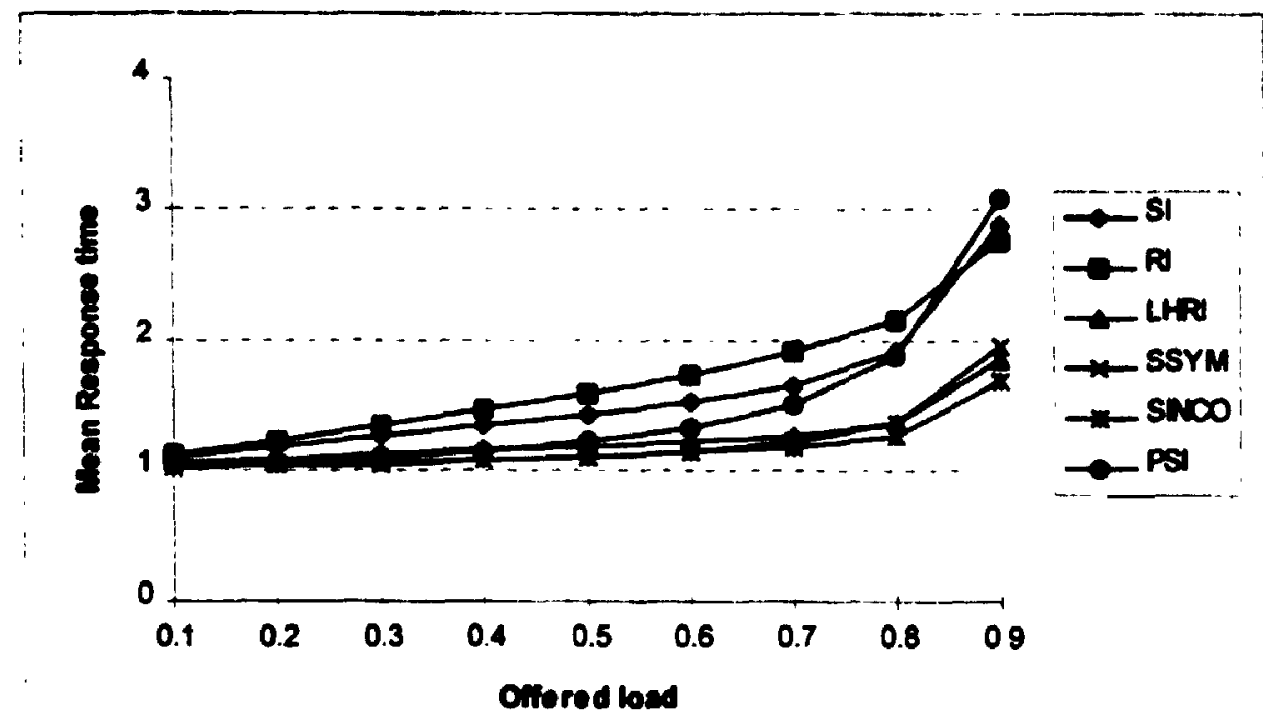

Figure 3.2(a) Mean response time as : function of offered load $\mathrm{N}=32$ nodes, $\mu=1, \lambda$ is varied to vary system load, $T_{1}=T_{h}=1, P_{1}=3, B=4$, transfer cost $=1 \%, T_{3}=2$ for $S I, T_{s}=1$ for $R I, T_{m}=2$ for $S I$ and $R I ; T=0$ for PSI and $T=I$ for SSYM, $C_{2}=C_{8}=1, T_{\text {wat }}=1, T_{\text {frue }}=0.2 . T_{\text {pard }}=0.4$ for PSI).

1. At high system loads, the performance of the local hierarchical (LHRI), stable symmetrically-initiated adaptive (SSYM) and single coordinator (SINCO) policies is substantially better than that of the sender-initiated (SI), receiver-initiated (RI) and periodic symmetrically-initiated (PSI) policies. SINCO gives the lowest mean response time at $70 \%$ to $90 \%$ loads, while SSYM and LHRI perform equally well over this range of loads.

2. The performance of PSI is superior to that of SI and RI except at $90 \%$ load. In the previous literature, PSI performed better than SI and RI even at 90\% load [Ben94]. The poor performance is due to the different threshold values used in these experiments. In these experiments, $\mathrm{T}$ is set to be zero for PSI; that is, it is a sender if the queue's length 
is greater than one (load.i $>T+1)$ and it is a receiver if it is idle (load. $i<T+1)$.

However, the threshold $T_{m}$ for $\mathrm{SI}$ is two which means that a node is a sender if the sum of the queue lengths is greater than two (job queue + job-wait queue length $>2$ ). In other words, there are fewer receivers under PSI, where $T=0$, than in SI because most of the nodes will have at least one job at $90 \%$ load. In this circumstance, job transfers initiated by sender nodes are substantially reduced in PSI and the system is moving towards no load sharing at this load. On the other hand, the timer period also reduces the chance for a receiver node to acquire a job from a sender node, as the duration of a node to be a receiver at this load is relatively shorter than that at a lower load. Thus, the $\mathrm{RJ}$ component of PSI cannot be as effective as the RI at this load. Therefore, the performance of PSI is worse than that of all other policies at $90 \%$ load. This shows that PSI is very sensitive to the threshold and timer period parameters.

3. At low to moderate loads, SSYM, LHRI, SINCO are the best policies among the six load sharing policies. PSI and SINCO show similar performance at the range of $10 \%$ to $40 \%$ system loads. The performance of $\mathrm{SI}$ is only comparable to that of SINCO at a very low load (10\%). Beyond $40 \%$ load, the mean response time of SI is at least $24 \%$ higher than that of SINCO. At this range of loads, RI has the poorest performance.

Figure 3.2(b) shows the mean response time of the sender-initiated policy, receiverinitiated policy, receiver-initiated policy with re-initiation period of one, and the local hierarchical receiver-initiated policy. The results for the sender-initiated policy (SI) and the receiver-initiated policy (RI) match those in the literature [Eager 86a, Dan 95a]. The 
performance of $\mathrm{SI}$ is marginally better than $\mathrm{RI}$ at $10 \%$ to $80 \%$ loads but $\mathrm{RI}$ performs better than SI at $90 \%$ load. At low to moderate loads. it is much easier for a sender node to find a receiver node as most of the nodes are likely to be lightly loaded or idle. Thus any job that would increase the job queue length beyond the threshold value $T_{m}$ would very likely be transferred almost immediately (i.e. after finishing probing) when SI is used.

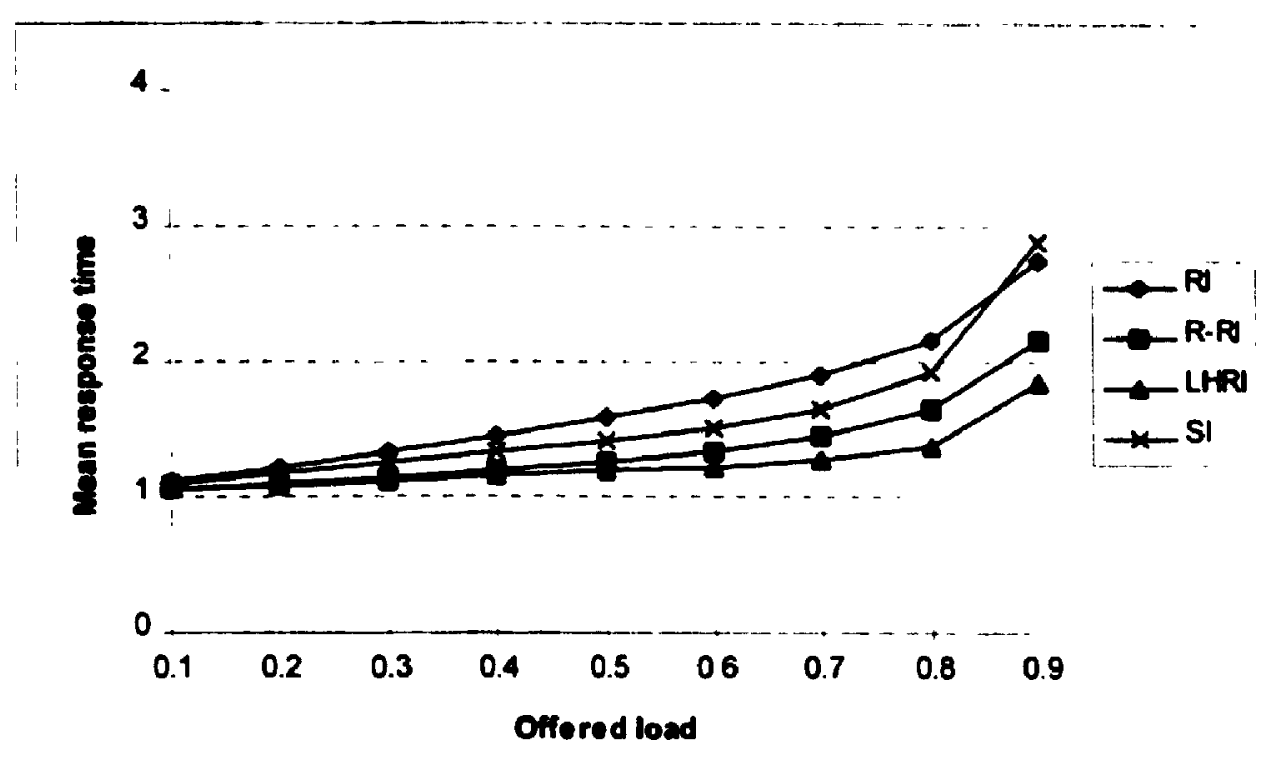

Figure 3.2(b) Mean response time as a function of offered load (RI, R-RI. SI, LHRI) $(\mathbf{N}=32$ nodes, $\mu=1, \lambda$ is varied to vary system load, $T_{1}=T_{h}=1, P_{1}=3 . B=4$, transfer cost $=1 \%$ and $T_{s}=2$ for $S I$ and $T_{s}=1$ for $R I, T_{m}=$ 2 for $\mathrm{SI}$ and $\mathrm{RJ} ; \mathrm{T}_{3}=1, T_{\mathrm{m}}=1$ for $\mathrm{R}-\mathrm{RI}, \mathrm{C}_{\mathrm{a}}=\mathrm{C}_{\mathrm{s}}=1$ ).

At $90 \%$ load, most of the nodes are not likely to be lightly loaded so the probability of successfully locating a receiver is low. The fruitless probing for a receiver further increases the system load. As the system load increases, more CPU time is wasted for such probing process (Note that not only the probing node CPU time is wasted but also that of other probed nodes is wasted). Thus, the performance of SI deteriorates rapidly at this load. 
However, the receiver-initiated policy with a reinitiation period of 1 performs better than $\mathrm{SI}$ and $\mathrm{RI}$ over the whole range of system loads. This is due to increased load sharing generated by the re-initiation of probing process. In R-RI a job transfer may not be immediate when a node becomes a sender but the delay would be shorter than that in RI, as more idle nodes are looking for senders with re-initiation of probing process (without reinitiation these nodes may still sit idle). Moreover, it is relatively easier to locate a sender in $R-R I$ than in RI. This is because $T_{m}$ is set to be one in R-RI. In addition, unlike SI no CPU time is wasted on probing while there are jobs to be processed under R-RI. Thus, probe reinitiation increases the number of job transfers with no additional burden on sender nodes and improves the performance of $\mathrm{RI}$. This performance improvement is shown by the performance of R-RI which reinitiates the probing process when a preset time period elapsed.

It may be noted that LHRI is the best load sharing policy among the policies shown in Figure 3.2(b) over the whole spectrum of system loads. This policy excels all these primitive load sharing policies for the following reasons. First, the load distribution activity of LHRI is receiver-initiated that performs well at high loads. Second, it allows the reinitiation of the search for sender that reduces the waste of computing resources at idle nodes. More importantly it maintains the state of nodes as effective as SINCO by utilizing a hierarchical structure. The following two paragraphs discuss the characteristics of the best three load sharing policies. 
The local hierarchical receiver-initiated policy (LHRI), the stable symmetricallyinitiated adaptive policy (SSYM) and the single-coordinator policy (SINCO) give low mean response time. The performance difference among these policies is within a narrow range over the whole range of system loads under examination. At moderate to high loads. SINCO offers the best performance. However. SINCO yields no better performance than SSYM at low loads. This is because SINCO does not include a sender-initiated component so load sharing activities are reduced at low loads. In other words, jobs have to wait longer to be transferred from busy nodes in SINCO. LHRI has a higher mean response time than SSYM except at $90 \%$ load. LHRI performs worse than SINCO over the range of system loads. This is because LHRI involves more overhead than the SINCO and the LHRI could not possibly be more effective than SINCO in selecting a sender. In SINCO, all nodes consult only a single node which maintains the state of the all nodes in a system. On the other hand, in the hierarchical policy each coordinator which maintains only the state of some nodes in the system is consulted by a subset of nodes. Each search request may need to flow through several coordinators thus may involve more overhead. At $90 \%$ system load, LHRI offers a $10 \%$ higher mean response time than that of SINCO while SSYM has a $15 \%$ higher mean response time than that of SINCO.

Both PSI and SSYM have a receiver-initiated component and a sender-initiated component. PSI and SSYM offer similar performance at low to moderate loads. However, when system loads increase, the gap between PSI and SSYM widens rapidly. This is because SSYM is more effective in controlling the sender-initiated probes which are 
unlikely to be successful at high loads. In addition, there are fewer receiver-initiated probes in PSI than in SSYM that reduce job transfers from sender nodes. This is because in PSI a receiver initiates a probing process only when the timer period has elapsed and the queue length is zero, not only when a job leaves an idle node. Thus, the performance of PSI degrades substantially at high loads.

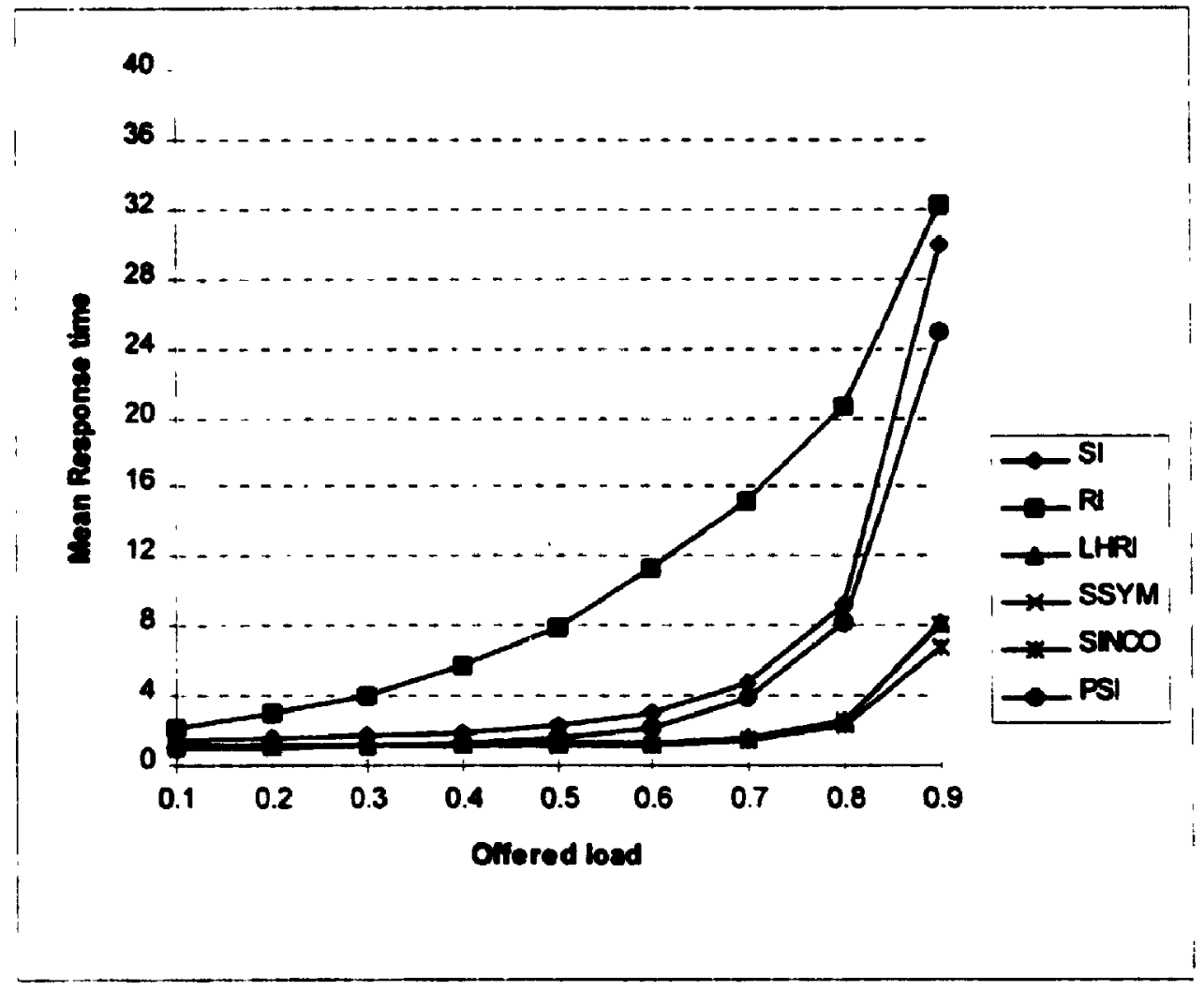

Figure 3.3 Mean response time as a function of offered load $\mathrm{N}=32$ nodes, $\mu=1, \lambda$ is varied to vary system lond. $T_{1}=T_{h}=1, P_{1}=3, B=4$, transfer cost $=1 \%$ and $T_{3}=2, T_{m}=2$ for $S I$ and $R I ; T=0$ for PSI and $T=1$ for SSYM, $C_{2}=C_{3}=4, T_{\text {mat }}=1, T_{\text {filue }}=0.2, T_{\text {period }}=0.4$ for PSI).

Figure 3.3 shows the mean response time as a function of offered system load with both $C_{a}$ and $C_{s}$ are fixed at 4 . The performance difference among policies are larger than that in Figure 3.2(a). The mean response time of all policies examined increases when both $\mathrm{C}_{\mathrm{s}}$ and $\mathrm{C}_{\mathrm{a}}$ are fixed at 4 . At high loads both the sender-initiated policy (SI) and the receiver- 
initiated policy (RJ) performs poorly, since the sender-initiated policy is sensitive to $C_{\text {s }}$ while the receiver initiated policy is sensitive to $C_{a}$ (that have also been shown in [Dan95a]). However, the sender-initiated policy performs better than the receiver-initiated policy at the whole range of system loads examined. This is because the sender-initiated policy allows more job transfers than the receiver-initiated policy at low to moderate loads and $\mathrm{RI}$ is relatively more sensitive to variance in inter-arrival times (see Section 3.4 .2 for details on performance sensitivity to variance in inter-arrival times).

The LHRI, SNCO nd SSYM policies again provide low mean response times and differ in a narrow range. These policies are stable even when there is high variance in both service times and inter-arrival times for the whole spectrum of system loads. As the system load increases, SINCO is better. At $90 \%$ offered system load, the mean response time of LHRI is $21 \%$ higher than that of SINCO and the mean response time of SSYM is $20 \%$ higher than that of SINCO.

The performance of PSI is significantly better than the receiver-initiated policy and marginally better than the sender-initiated policy. The marginal performance difference between PSI and SI is due to two factors. First, PSI has the sender-initiated component as well as the receiver-initiated component, which together increase load sharing activities and job transfers. Second the sender-initiated component of PSI is not as sensitive to variance in service time as SI, since the sender-initiated component of the location policy of PSI is triggered not only every time a node is overloaded but also when the predetermined period has elapsed for that node. Therefore, the sensitivity of PSI to 
variance in service times is reduced. However, the performance of PSI is still not comparable with that of SSYM, LHRI and SINCO at high system loads and high CVs.

\subsubsection{Sensitivity to Variance in Inter-Arrival Times}

This section considers the impact of inter-arrival coefficient of variation $C_{2}$ on the performance of the six load sharing policies. Figure 3.4 shows the mean response time of the six policies when the offered system load is fixed at $80 \%$ (i.e., $\lambda=0.8$ ) and service time coefficient of variation $C_{s}$ at 1 . All other parameters values are set as in Figure 3.2(a). From Figure 3.4, we can see that the best three policies, LHRI, SSYM, as well as SINCO in the previous sections, are less sensitive to variance in inter-arrival times than the other three policies, SI, RI and PSI. Increased inter-arrival time coefficient of variation $\mathrm{C}_{\mathbf{a}}$ means that the job arrival process at each node is clustered. The higher the $\mathrm{C}_{w}$, the more clustered the arrival process is. At a higher variance, jobs arrive as a larger batch at each node, which decreases load sharing activity generated by each node as the probability of finding a node with no job to execute is low. Eact. Ende must finish a larger batch of jobs before it can generate any probe. Thus, increasing the inter-arrival time coefficient of variation moves the system using RI policy towards no load sharing [Dan95a]. 
So it is not surprising that the basic receiver-initiated policy (RI) is the most sensitive to inter-arrival coefficient of variation. On the other hand. the c'. tered arrival of jobs increases the frequency of a node being a sender, and thus fosters increased load sharing activity in the sender-initiated policy. That explains why the sender-initiated policy is relatively less sensitive to variance in inter-arrival times than the receiver-initiated policy. The PSI exhibits similar behavinur as the sender-initiated policy, which is mainly due to the sender initiated component. It should be noted that LHRI, SSYM and SINCO are close in performance; however SINCO outperforms LHRI by 3\% to $15 \%$ and SINCO excels SSYM by $8 \%$ to $16 \%$ when $\mathrm{CV}$ is increased from 0 to 4 .

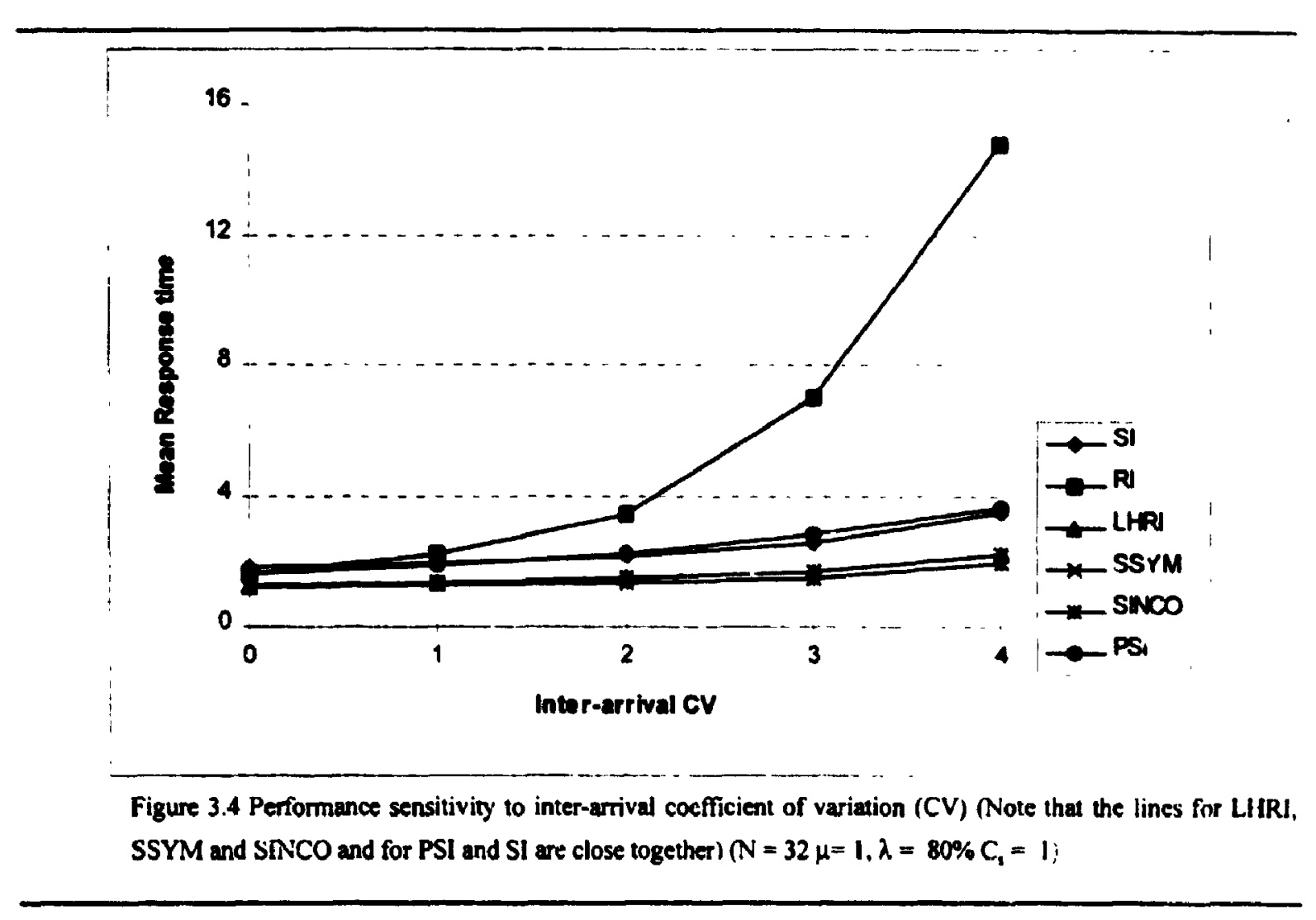




\subsubsection{Sensitivity to Variance in Service Times}

This section considers the impact of service time coefficient of variation $C_{s}$ on the performance of the six policies. In this context, it should be noted that the ser .ce time distribution of the data collected by Leland and Ott [Lel86] from over 9.5 million processes has been shown to have a coefficient variation of 5.3 [Kru88]. Figure 3.5 shows the mean response time when the offered system load is fixed at $80 \%$ (i.e., $\lambda=0.8$ ) and the interarrival time $\mathrm{C}_{\mathrm{a}}$ at 1. All oiher parameters are set as in Figure 3.2a.

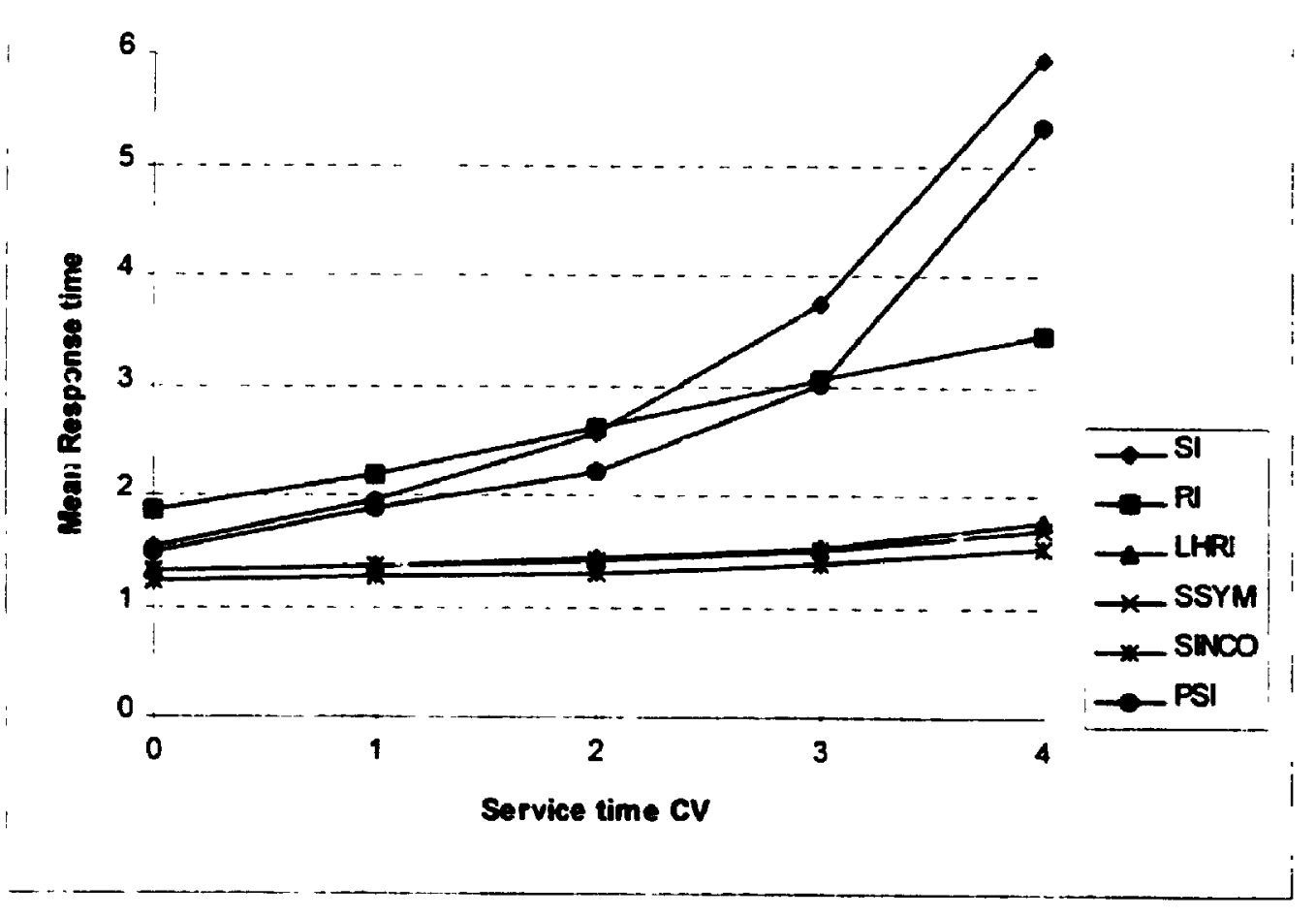

Figure 3.5 Performance sensitivity to service time coefficient of variation

(Note that the lines for LHRI and SSYM are close tugether. $N=32, \mu=1, \lambda=80 \%, C_{n}=1$, transfer cost $=$ $1 \% T_{\text {wall }}=1, T_{\text {ralse }}=0.2 . B=4 . T_{\text {period }}=0.4$ for PSI) 
The significant observations from Figure 3.5 are that SSYM. SINCO and LHRI are considerably less sensitive than PSI, RI, SI, and the SI is the most sensitive to variance in service time. With the FCFS node scheduling policy larger jobs tend to monopolize the processing power, which causes increased queue lengths. resulting in increased probing activity under the sender-initiated policy (SI). However, this increase in probing activity is useless because at high system loads the probability of finding a receiver node is low. On the other hand, RI, SINCO, LHRI. PSI and SSYM policies with the receiver-initiated component. can successfully locate a sender because the probability of finding an overloaded node is high. This results in better performance and reduced seısitivity of RI. PSI, SINCO LHRI and SSYM to variance in job service demand.

SINCO exhibits more superior behaviour, its response time only increases $20 \%$ while the mean response time of LHRI and SSYM increases about $33 \%$ and $30 \%$ respectively when the $C_{s}$ increases from 0 to 4 . These results further demonstrate that SSYM is effective in controlling unnecessary probing generated by sender nodes that reduces the sensitivity to service time coeffic ient of variation $C_{s}$. The PSl is less sensitive to service $\mathrm{C}_{\mathrm{s}}$ than $\mathrm{SI}$, even though it has a sender-initiated components. This is mainly due to the fact that the location policy of PSI is initiated only when the timer period elapsed and queue length was above some threshold value or below the threshold value. This reduces the amount of unnecessary probing generated by sender nodes at high service time CV, many nodes dre monopolized by large jobs and it is difficult for senders to locate a receiver. 
On the other hand, the timer period also reduces the benefit from receiver-initiated load sharing activities. Thus, when $C_{2}=4$, basic $R I$ shows better performance than PSI.

\subsubsection{Performance Sensitivity to Probe Limit}

This sections shows the sensitivity to probe limit $P_{1}$ of three policies namely, SI, RI and SSYM (Note that other policies do not require probing or only probe once each time a location is invoked). Figure 3.6 shows the mean response time versus probe limit. In this experiment, the probe limit is varied from 1 to 10 . The value of both $C_{2}$ and $C_{5}$ is set to four and the offered load is fixed at $85 \%$.

Increasing the probe limit for SSYM does not result in much performance improvement after the probe limit reaches 2 . As we can see, there is only marginal increase in performance when the probe limit change from 2 to 4 and it levels off when the probe limit reaches 4 . $\mathrm{RI}$ and $\mathrm{SI}$ are more sensitive to the probe limit. The performance gain of SI and $\mathrm{RI}$ from increasing the probe limit from 3 to 10 is $20 \%$ and $70 \%$ respectively. $\mathrm{RI}$ shows better performance than SI when probe limit reaches 10 . The results show that $\mathrm{RI}$ is more sensitive to probe limit than SI. Since the rate of probing generated at each receiver node is very small at high system loads and high inter-arrival coefficient of variation as explained earlier, it is important for a receiver to locate a sender at each probing process in order to yield good performance. The probability of success in probing for a sender increases with the value of probe limit. Thus, the increase in probe limit improves the performance of $\mathrm{RI}$. 
On the other hand, the further increase in probe limit does not improve the performence of SI because most of the nodes are monopolized by large jobs and very ur..ikely to be a receiver and the chances of a probing process to succeed would not increase by increasing the probe limit. In fact, increased probe limit causes more overhead and degrades the performance of SI.

The result of SSYM, although obtained at a higher system load and higher CV values, is similar to those in [Shi90]. As mentioned in [Shi90], the system state is accurately reflected in the state lists at each node that ensures the high probability of successful probing for partners. Since SSYM does not rely on the value of probe limit to yield high performance. it is scalable to large distributed system.

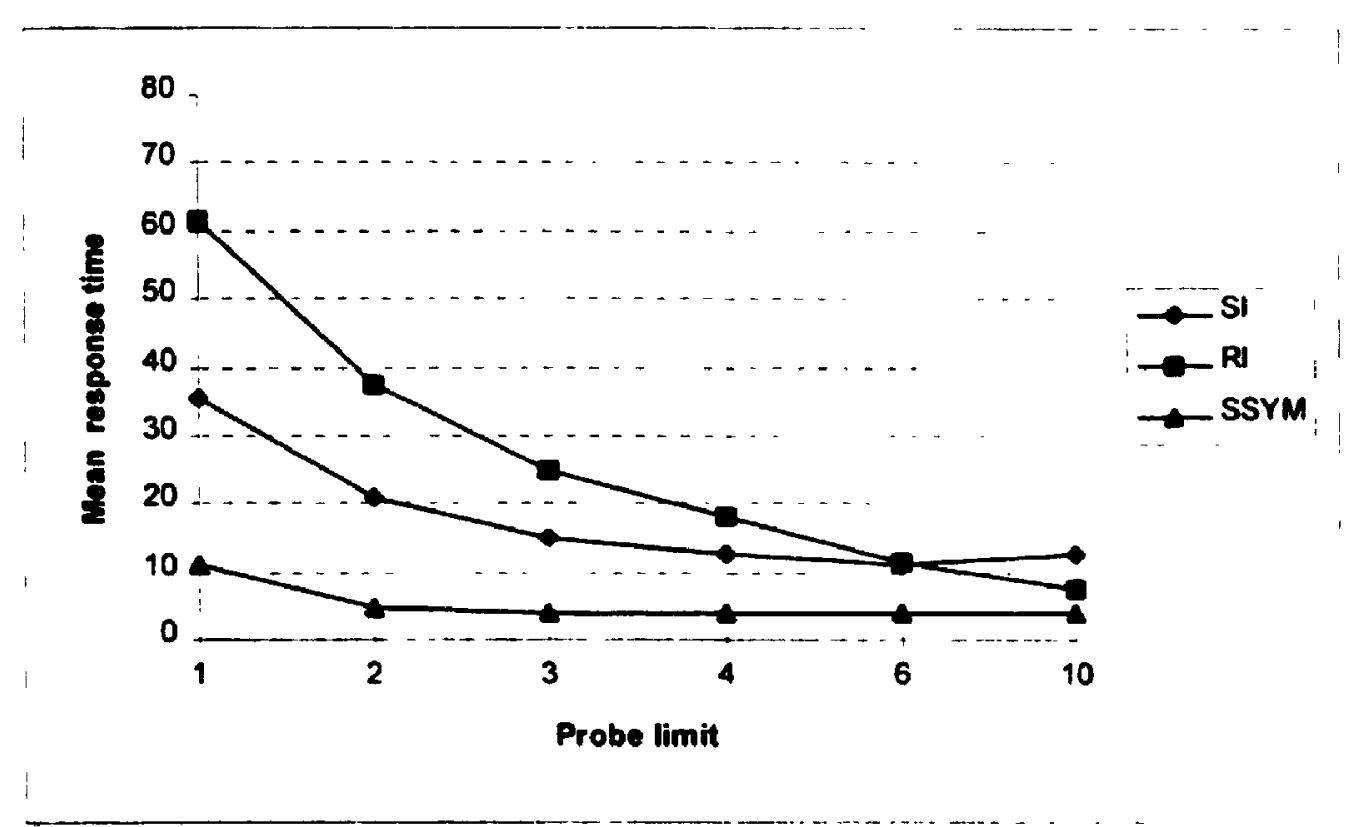

Figure 3.6 Performance sensitivity to probe limit $\left(N=32, \mu=1, \lambda=85 \%, C_{4}=C_{3}=4\right.$, transfer cost $=1 \%$ ) 


\subsection{Two Variations of the Hierarchical Receiver-Initiated Policy}

A hierarchical receiver-initiated policy is described in Section 2.12 and its performance is compared with other load sharing policies in the previous sections. This section describes two variations of hierarchical policies, namely the global non-recursive and global recursive hierarchical policies and the performance of three hierarchical policies is studied. They are named global policies in the sense that no preference is given to nodes in local clusters for load sharing. The hierarchical policy described in Chapter 2 is named as local hierarchical load sharing policy because it encourages local load sharing.

\subsubsection{Global Hierarchical Load Sharing Policies}

The local hierarchical policy promotes local load sharing. Figure 2.1 indicates that the node NO, a sender, is not known beyond the cluster of N0 and N1 nodes. Thus, if for example there is a receiver node outside the cluster looking for a sender, the existence of N0 as a sender node for load sharing is not known to that receiver node. Note, however, that this is a transient situation as the receiver node N1 eventually gets a job transferred from node N0. This job transfer might be delayed for some time depending on the re-initiation period. If node N0 remains a sender after the job transfer, this information will be made available to other nodes in the system.

The net effect is that the local policy sometimes delays informing other nodes of the existence of a sender node by giving priority to local load sharing. This type of load sharing is preferred when the system consists of L.AN clusters connected together by a WAN. If, on 
the other hand, the system is simply a single cluster of nodes connected by a LAN. performance improvements can be obtained by reducing this delay. This section describes two variants of local hierarchical policy that achieve this objective.

\section{Q)}

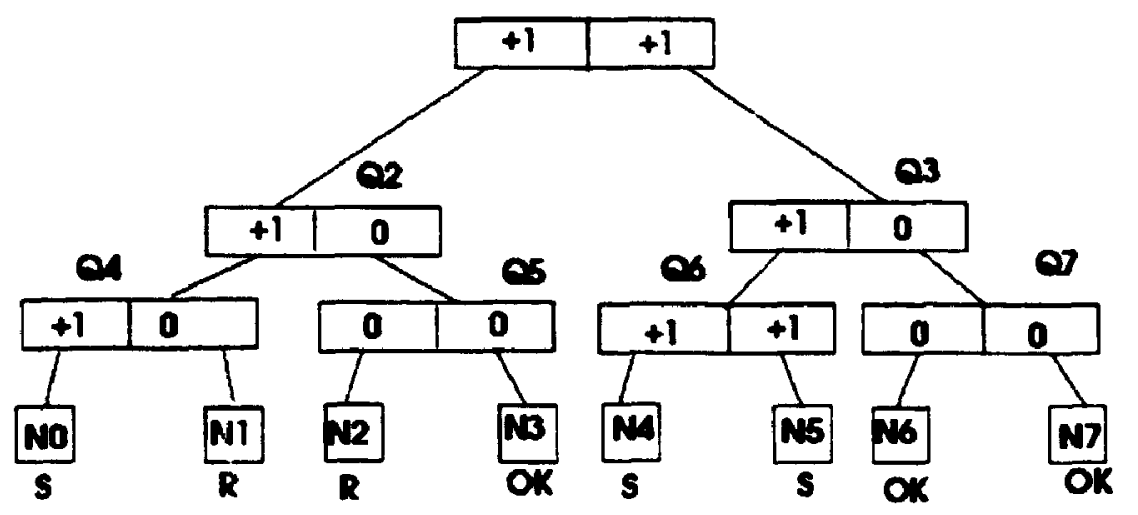

Figure 3.7 An example of global hierarchical organization for an 8-node system with branching factor $\mathbf{B}=\mathbf{2}$

Like the local policy, load sharing is initiated at a receiver node and state information of nodes is stored in a hierarchy in global policies. The two global policies differ from the local policy in the type of information maintained in the hierarchy and in handling the 'false-sender' scenario (described below). In the two global policies the existence of a sender node is publicized as widely as possible so that any receiver node in the whole system can initiate a job transfer. This is suitable for systems that do not have an inherent hierarchical structure in the communication network (as indicated before, the system is assumed to be a single cluster of nodes).

Figure 3.7 shows the information maintained in the hierarchy in the global policies for the same node states as in Figure 2.1. It can be observed from Figure 3.6 that the hierarchy only maintains two states: non-sender (receiver or $\mathrm{OK}$ ) and sender states. The 
sender state and non-sender state are indicated as 1 and 0 respectively. Under this scheme, the meanings of the state information kept in the tree nodes are: if there is at least one sender in that branch, a 1 is stored to indicated the fact; otherwise a 0 is stored. For example, the first value in Q2 is 1, which indicates the fact that there is at least one sender in the left subtree.

In these global policies and the local hierarchical policy described in Chapter 2, a receiver initiates load distribution by requesting a job from a sender node. This request flows through the hierarchy (up the tree and then down the tree) and finally terminates at the sender node (if there is one) or at the root node if there is no sender in the whole system. The sender node, upon the receiving a request from a receiver, directly replies to that node either by sending a job to it or a 'false-sender' message if its state has changed from sender to $\mathrm{OK}^{6}$. This is referred to as the "false-sender" scenario. In the global recursive policy when a request message ends up with a false sender, the search process continues by backtracking until a "true sender" is found". Thus, the global recursive policy improves the performance at the expense of increased message overhead. The performance of these hierarchical policies is presented in the next subsections.

- The state information in the hierarchy might be out of date temporarily because of message delays asociated with updating the hierarchy.

7 Unless there is no sender in the entire system; in this case, the root tree node sends a "no job" message to the originator of the request. 


\subsection{Performance Comparison of Hierarchical Load Sharing Policies}

This section presents the results of the simulation experiments on three hierarchical load sharing policies: the local hierarchical policy (LHRI), the global non-recursive hierarchical policy (GNHRI), and the global recursive hierarchical policy (GRHRI). The system and workload models used are the same as those described in Section 3.2.

\subsubsection{Principal Performance Comparison}

Figure 3.8(a) and Figure 3.8(b) plot mean response time of the three hierarchical policies versus offered system load. As in the Section 3.4 , the offered system load is defined as $\lambda / \mu$. Since $\mu=1$ for all the experiments in this section, the offered load is equal to $\lambda$. The service time coefficient of variation $\left(C_{s}\right)$ and the inter-arrival coefficient of variation $\left(C_{2}\right)$ are set to 1 in Figure 3.8(a). Both $C_{s}$ and $C_{a}$ are set to 4 in Figure 3.8(b). All other parameters are set to the default values mentioned in Section 3.4.

Figure 3.8(a) shows that at low system loads, the performance difference of three hierarchical policies is very small. The local hierarchical policy (LHRI) performs marginally worse than the two global policies at low to moderate loads and the performance difference becomes noticeable at $30 \%$ load. At low to moderate loads there are only a few sender nodes in the system and the time delay for a sender to be known to the whole system under LHRI is longer than that under GNHRI and GRHRI. In other words, the probability for a receiver node under LHRI to acquire job from a sender node is much less than for a 
receiver node to acquire a job from a sender under the two global policies. The performance difference between GNHRI and GRHRI is even smaller and it is noticeable only at $\mathbf{5 0} \%$ and $\mathbf{9 0 \%}$ loads. The GNHRI yields insignificantly better performance than GRHRI at low to moderate loads due to smaller overhead incurred without back tracking in GNHRI (this fact is only evident at $50 \%$ system load). Since there are not many sender nodes in the system, additional searches may be useless in GRHRI. However, at high system loads (from $80 \%$ to $90 \%$ ), the performance GRHRI is marginally better than that of LHRI and GNHRI. The performance of GNHRI and LHRI is still very close at this same loads.

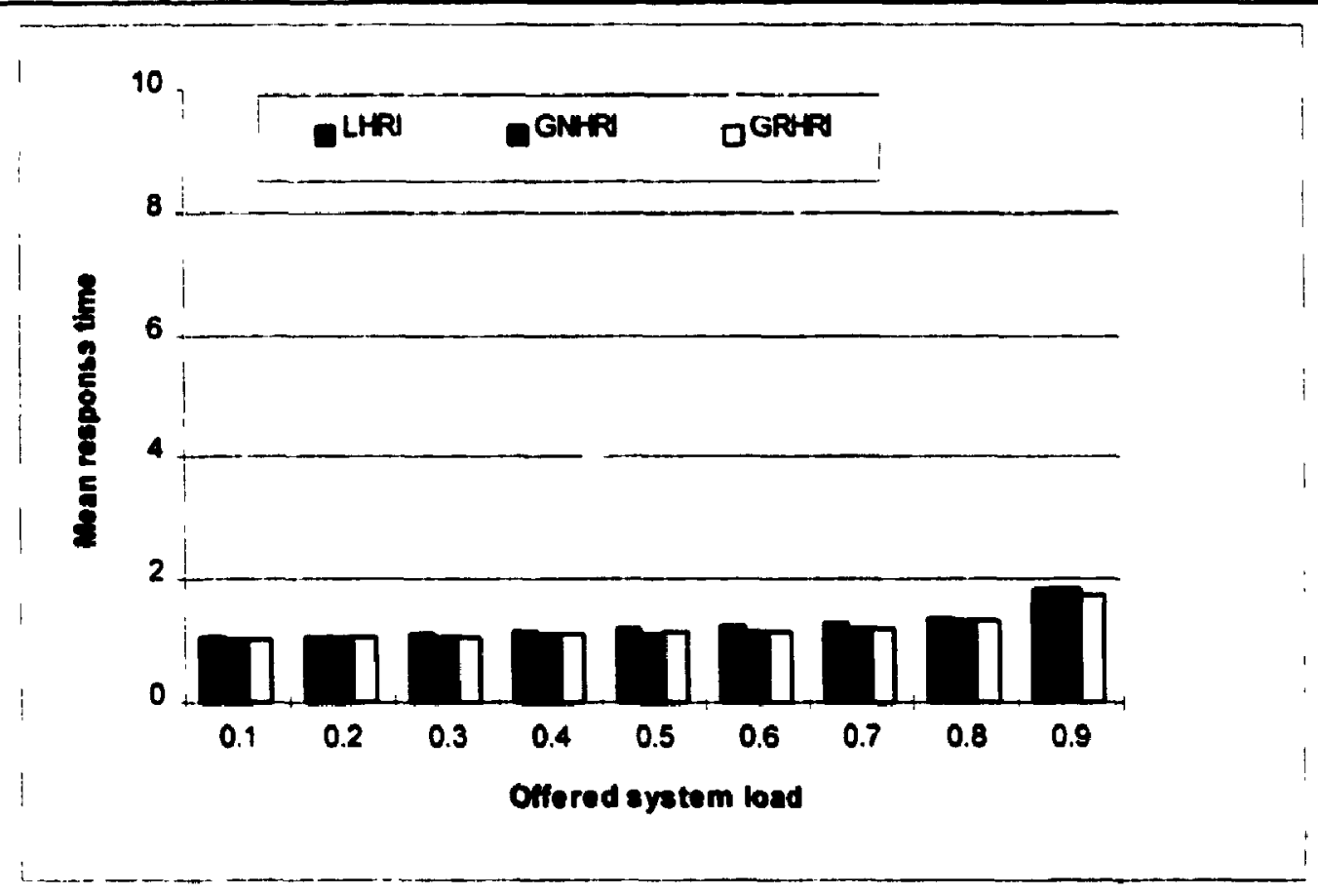

Figure 3.8(a) Mean response time versus offered system load $\left(N=32, T_{1}=T_{h}=1, C_{a}=C_{s}=1, T_{\text {wat }}=1\right.$, $T_{\text {false }}=0.2, B=4, \lambda$ is varied to vary offered system load, $\mu=1$ ) 
The mean response time of GRHRI is about $5.4 \%$ and $4.5 \%$ less than that of GNHRI and LHRI respectively, at $90 \%$ load. The better performance of GRHRI is due to the use of the back tracking method in searching for a sender node. That is, when a search request encounters a "false sender" (an unsuccessful search), the parent tree node will be notified immediately. However, in the LHRI and GNHRI policies the receiver must wait for a re-initiation of 0.2 unit once the search encounters a "false-sender". Thus, a receiver node under GRHRI has a relatively higher probability of acquiring a job than that under both GNHRI and LHRI.

Figure 3.8(b) shows similar performance patterns, in which at low to moderate system loads their performance difference is marginal and at high loads GRHRI once again yields better performance. However, at high system loads the performance difference among policies shown in Figure 3.8(b) is larger than that shown in Figure 3.8(a). GNHRI and LHRI yield almost the same mean response time at high system loads. These results show that back tracking is necessary in order to further improve the performance of a global hierarchical policy at high system loads, and at high variance in service times and in interarrival times; and the additional overhead is worth spending in order to obtain such a improvement. The recursive global (GRHRI) policy gives about $12 \%$ better performance than both GNHRI and LHRI at $90 \%$ load in Figure 3.8(b). The performance sensitivity of the three hierarchical policies to service time and inter-arrival time coefficients of variation (CVs) is presented in the next section. 
10

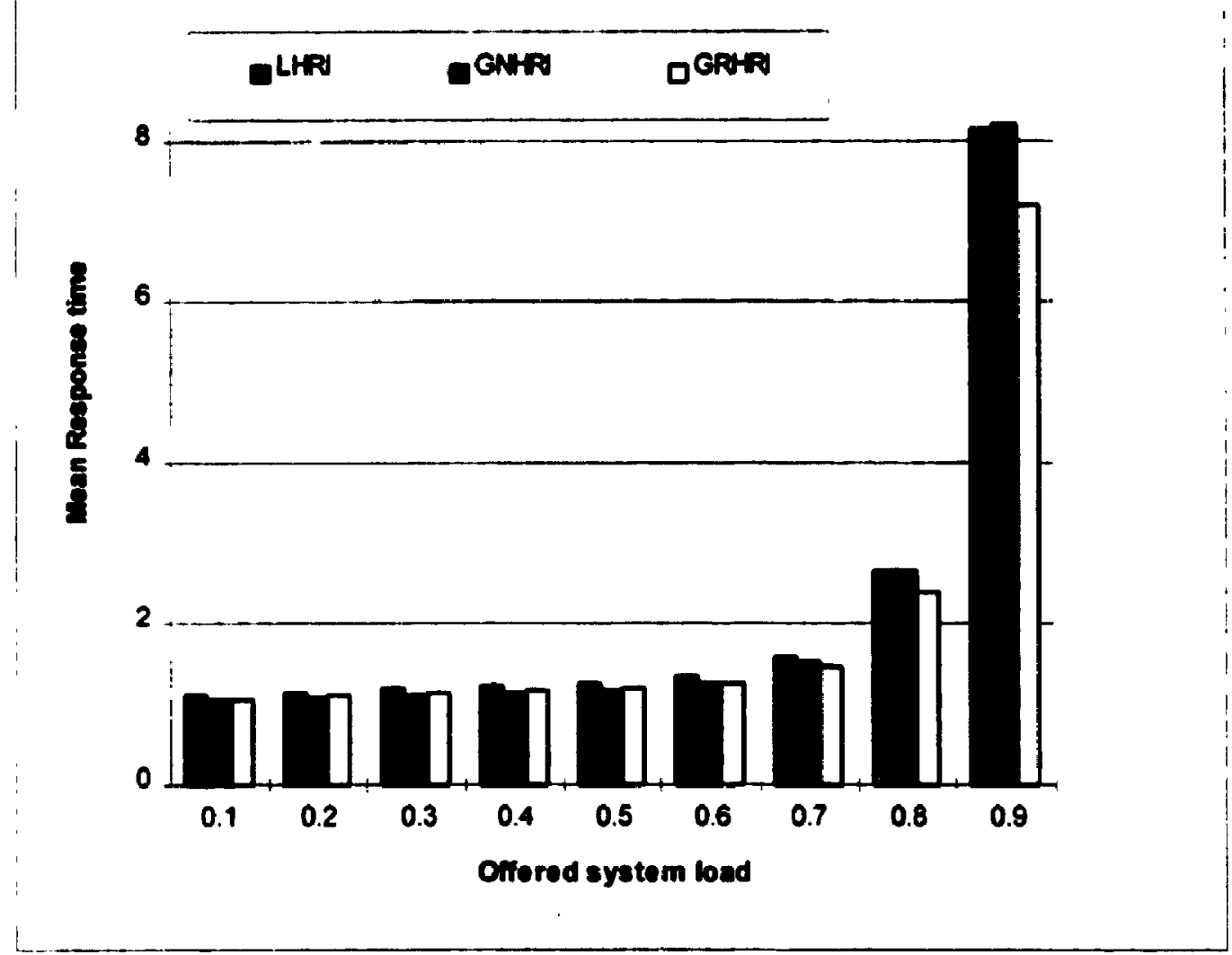

Figure 3.8(b) Mean response time versus offered system load $N=32, T_{1}=T_{h}, C_{2}=C_{3}=4, T_{\text {wer }}=1$, $T_{\text {fula }}=0.2, B=4$, is varied to vary offered system load

\subsubsection{Sensitivity to Variance in Service Times and Inter-Arrival Times}

Figure 3.9 shows the performance sensitivity of three hierarchical policies to service time coefficient of variation $\mathrm{C}_{s}$. The offered system load of this experiment is fixed at $80 \%$ and all other parameters are the default given in Section 3.6. We can see from this figure that the mean response time of three hierarchical policies increases as $\mathrm{C}_{s}$ increases. They all exhibit a very stable behaviour even at service time $\mathrm{CV}$ of 4 . That is, there is no immense increase in inean response time. The performance of GRHRI is relatively less sensitive to 
service time CV than that of the other two hierarchical policies. Th: performance difference between GRHRI and LHRI and between GRHRI and GNHRI increases with service time CV. The performance difference between GNHRI and LHRI is more or less the same for all values of $\mathrm{CV}$ tested.

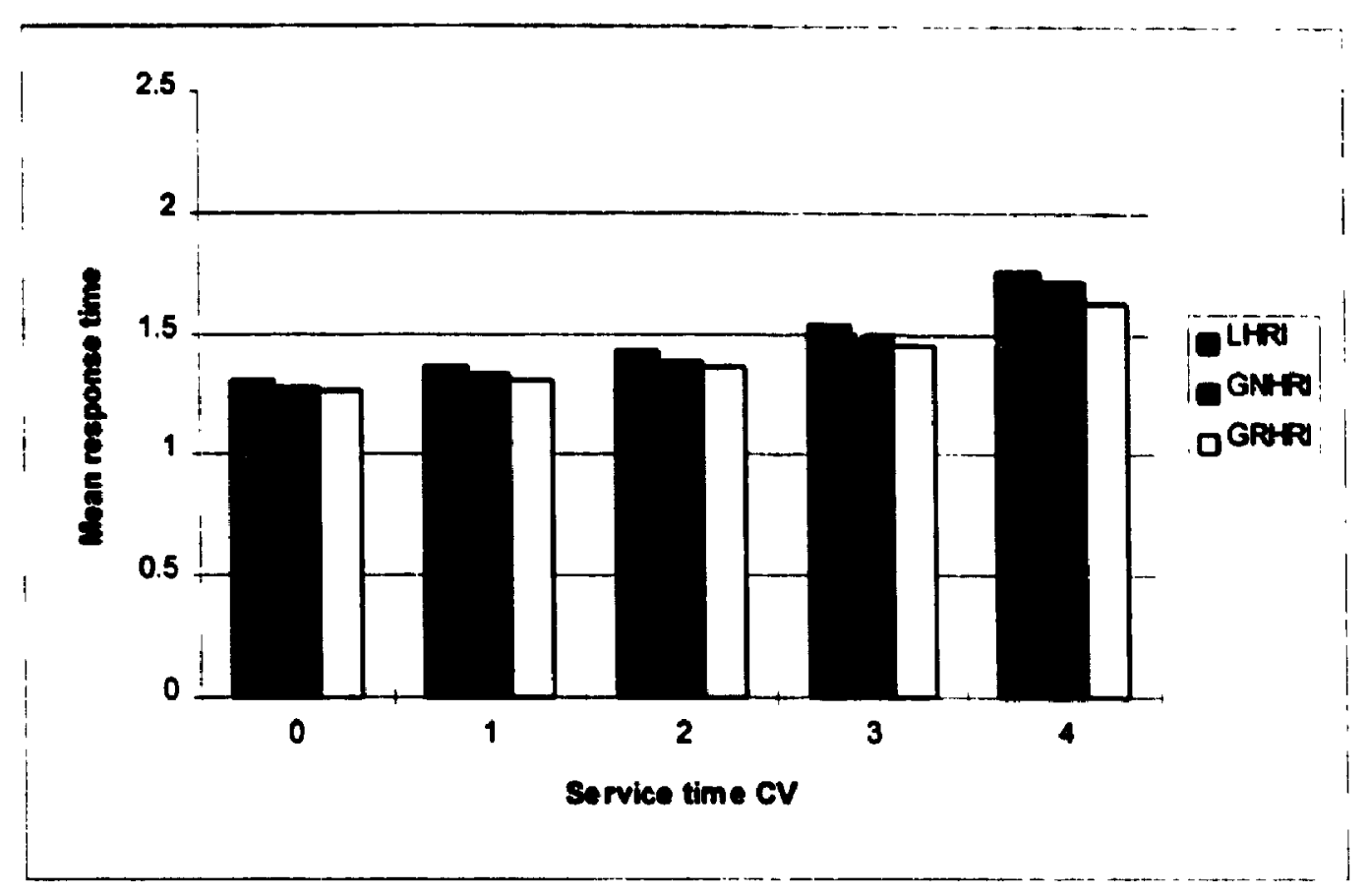

Figure 3.9 Impact of variance in service times $\left(C_{a}=1, B=4\right.$ for all policies, $\left.T_{h}=T_{1}=1, \lambda=0.8 \mu=1\right)$

Figure 3.10 shows the impact of the inter-arrival time $\mathrm{CV}$ to the mean response time of the three hierarchical policies. All policies are relatively more sensitive to the interarrival time CV than to the service time CV. This is due to the fact that all hierarchical load sharing policies are based on receiver-initiated activities, which have been shown (in Section 3.4.2) to be more sensitive to the inter arrival time CV than to the service time CV However, with the hierarchical organization the increase in mean response time of GRHRI 
is only about $54 \%$ when the inter-arrival time $\mathrm{CV}$ is changed from 1 to 4 . This is significantly lesser than the basic receiver-initiated policy $(\mathrm{RJ})$, which exhibits more than $570 \%$ increase in mean response time when the $\mathrm{CV}$ is alternated from 1 to 4 (see Figure 3.4).

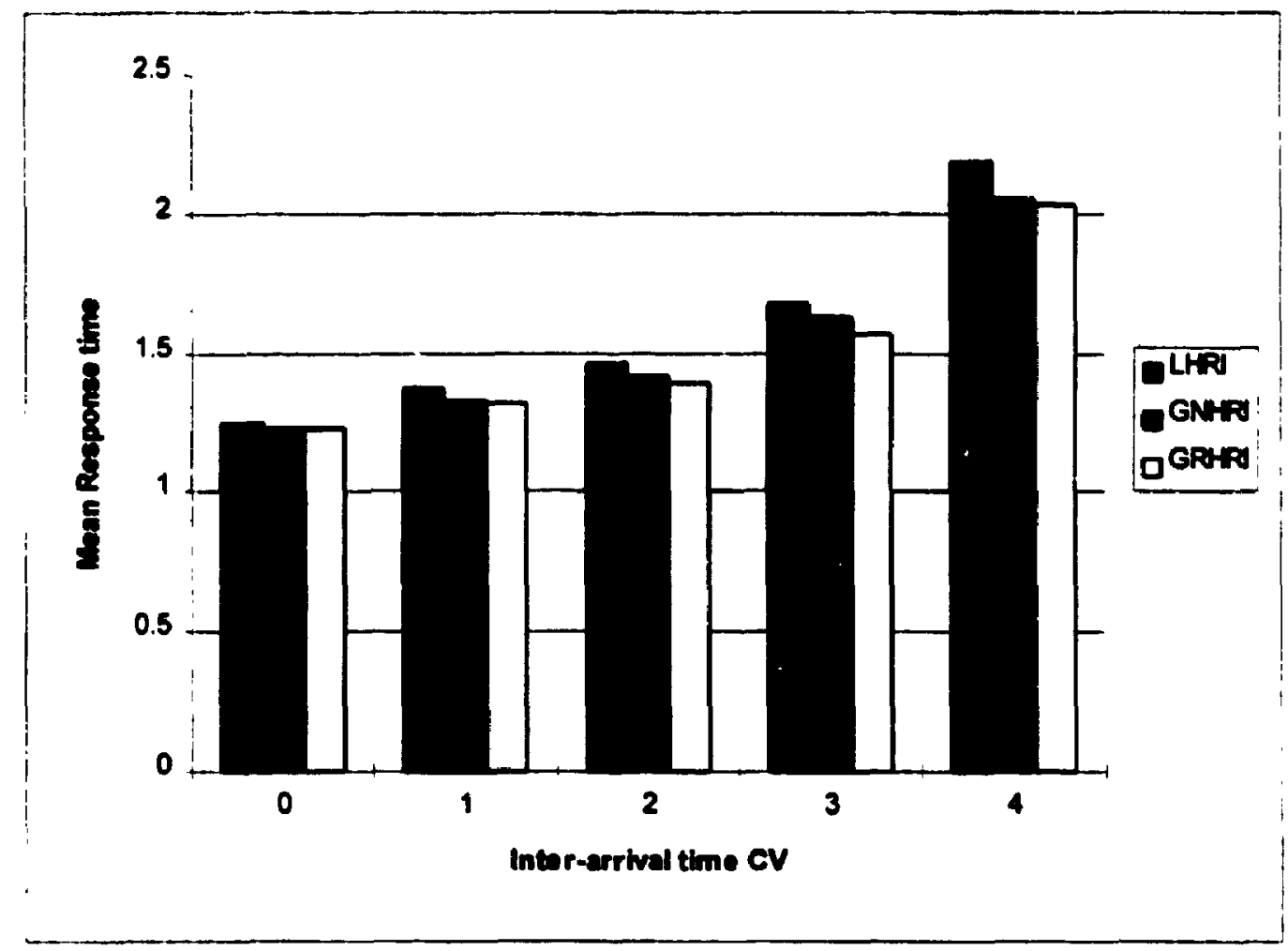

Figure 3.10 Performance sensitivity to inter-arrival $C V\left(N=32, T_{1}=T_{h}=1, B=4, C_{s}=1 \mu=1 \lambda=0.8\right.$ )

\subsubsection{Performance Sensitivity to Branching Factor}

This section discusses the performance sensitivity of three hierarchical load sharing policies to the branching factor. In these simulation experiments, the branching factor is varied from 2 to 32 . As in previous simulation experiments, the $\mathrm{CV}$ of inter-arrival time and service time are both set to 4 . The default values are used for all other parameters. 
Figure 3.11 shows mean response time as a function of branching factor. The offered system load is set at $80 \%$ and the threshold $T_{h}$ and $T_{1}$ are both set to 1 . The reinitiation period for the "no-sender" and "false-sender" scenario is set to 1 and 0.2 respectively. We can see from Figure 3.11 that the mean response time of the three hierarchical policies decreases with the increasing branching factor. At the extreme case. where the branching factor is 32 , all three policies behave like the single coordinator policy. The hierarchical is reduced into a single node which maintains the state of all the nodes in the system and makes the load sharing decision based on complete system state information. In this case, the global recursive (GRHRI) yields marginally worse performance than the other two hierarchical policies. This is due to the fact that the back tracking for the "false-sender" scenario is fruitless. It is very unlikely that the hierarchy of a single node is not reflecting an up-to-date load information of the whole system.

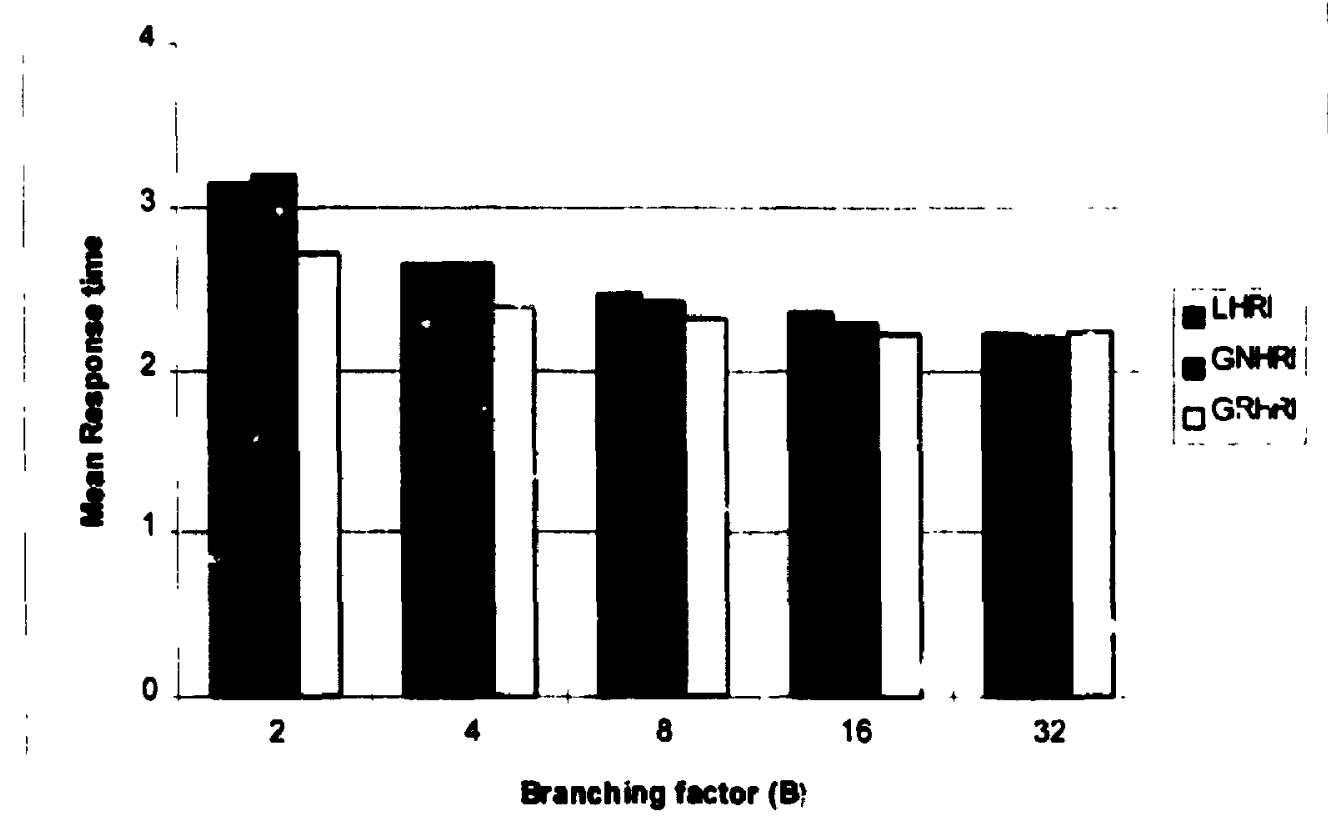

Figure 3.il Performance impact of branching factor on three hierarchical policies $\left(C_{2}=C_{1}=4, \lambda=0.8 \mu=1\right)$ 
However, when the branching is 2 , the global recursive policy with a back tracking mechanism yir Ids substantial performance improvement over the other two policies. The LHRI and GNHRI provide similar performances over the values of branching tested. In case of a branching factor of 2 , the performance of the local hierarchical policy is marginally better than the non-recursive global policy. This is because the local hierarchical policy encourages local sharing that reduces the chance of the "false sender" scenario when the branching factor is small, as the local receiver node is given higher priority for acquiring a job from a sender located in the same cluster than receiver nodes in other clusters.

However, in the non-recursive global policy, more CPU overhead is wasted on fruitless searches that fail because of "false-sender". For example, in Figure 3.7 when both $\mathrm{N} 1$ and N2 initiate a searct., only one of the two search requests can be satisfied using a global scheme (assumed N0 currently has only one extra nd the re-initiation period of N1 expires ). If the request of $\mathrm{V} 1 \mathrm{l}$ arr ves first, then $\mathrm{N} 2$ must wait for an re-initiation of 0.2 befol: initiating another search. This situation occurs more often when the branching factor is small. since there are not many senders for a tree-node to select from to avoid such a problem. In the local policy (Figure 2.1) the state of Q4 in Q2 is 0 and thus N2 request goes up along the hierarchical to the root and N2 eventually acquire a job from other sencier in other clusters. This is evident by the fact that, in general, at each level the average message handling rate of global non-recursive policy is higher than that of the local policy. The averal $\geq$ message handling rate of the hierarchical policies is presented in the next section. 


\subsubsection{Reduction in Message Handling Rate}

One of the goals of the hierarchical policy is to avoid the performance bottleneck problem caused by a single node in the single coordinator centralized policy. This section demonstrates that the bottleneck problem is reduced by using a hierarchical organization. Figure 3.12 and Figure 3.13 plot average message handling rate of tree nodes at a particular level as a function of offered system load (from $10 \%$ to $90 \%$ ). Figure 3.12 corresponds to the service time and inter-arrival time CVs of 1 and those in Figure 3.13 corresponds to both $\mathrm{CV}^{\mathrm{V}} \mathrm{s}$ of 4 . The branching factor of all policies is fixed at 4 .

The average message handling rate at each level $i$ is defined as:

number of messages received + number of messages ser.. out by all nodes at level $i$ number of tree-nodes at leveli ${ }^{*}$ number of jobs completed

In both figures, we can see that at any level the message handling rate of each hierarchical policy is less than that of the single coordinator policy. Note that in the single coordinator policy only the root node experiences this average message handling rate. The average message handling rate decreases and is more evenly distributed with increasing system loads. At high system loads, the number of receivers decreases and the search activit; decreases, thus reducing the search messages received and sent out by each treenode at each level. At low system loads (10\% to $30 \%)$, the root node of all policies including single coordinator has a similar handling rate, since most job requests must go up tc the top of the hierarchical as there are only a few senders in the whole system. 
However, at moderate to high system loads most of the searches can be satisfied by ieaf and intermediate tree-nodes, reducing the average message handling rate of the root node in all hierarchical policies. The middle level has the highest rate when the offered load is between $60 \%$ to $90 \%$, since at this range of loads most requests can be filled at the leaf tree-nodes and middle nodes but there are fewer number of them than that of the leaf treenodes. In other words, if there were equal number of middle tree nodes and leaf tree-nodes, the average rate would be more or less the same. At $80 \%$ load where both CVs are 1 , the average message handling rate of the root node, middle node and leaf node for all hierarchical policies is about $23 \%$ to $35 \%, 41 \%$ to $51 \%$ and $15 \%$ to $18 \%$ of the rate in single coordinator policy respectively. For both CVs of 4 with the same offered system load, the average message handling rate of the root node. middle nodes and leaf nodes for all hierarchical policies is about $23 \%$ to $30 \%, 37 \%$ to $50 \%$ and $14 \%$ to $18 \%$ of the rate in single coordinator policy respectively. 


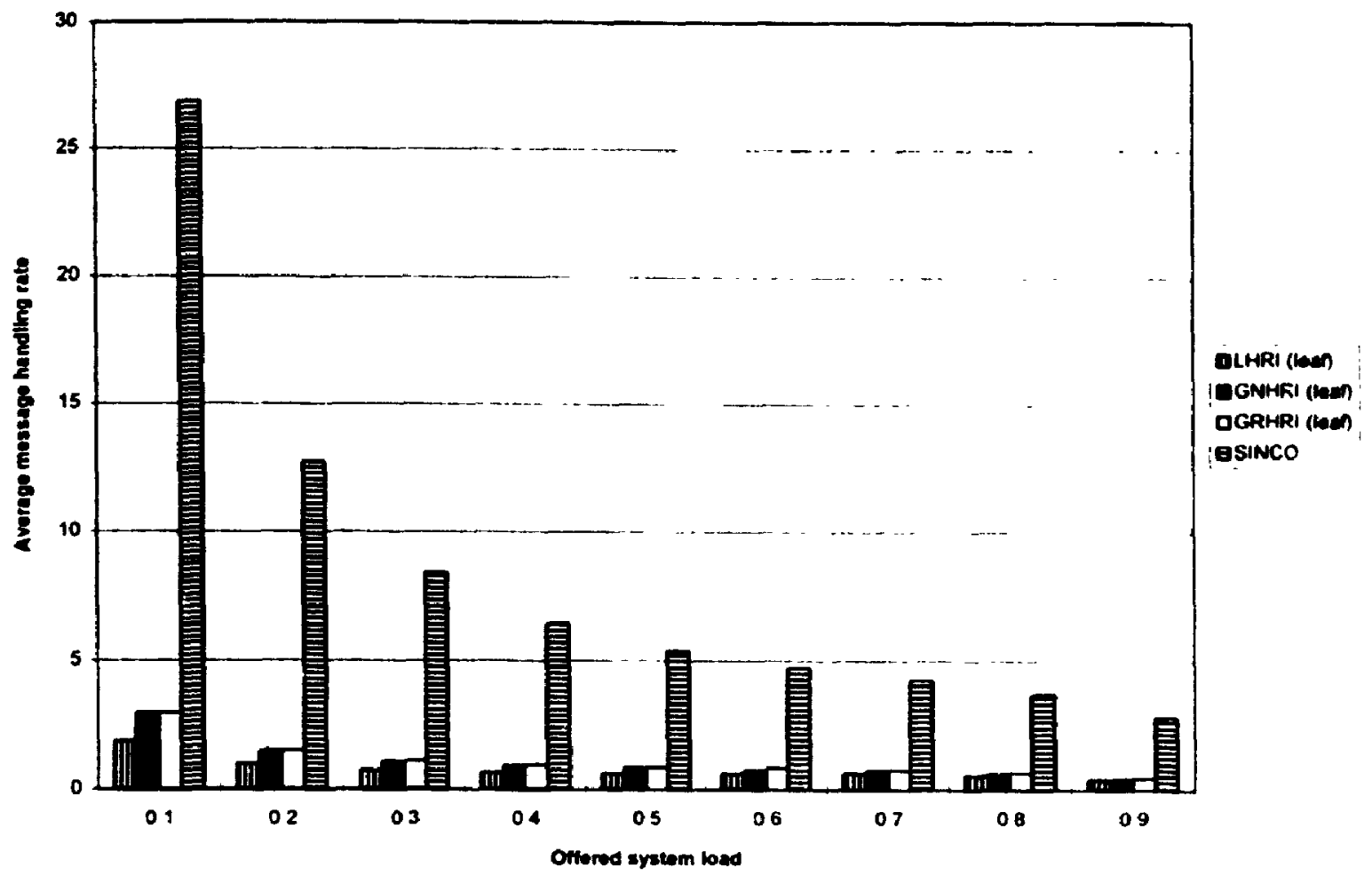

Figure 3.12(a) Average message handling rate at leaf level $\left(N=32 . B=4, C_{n}-C_{s}=1, \mu=1, \lambda\right.$ is varied to vary offered system load)

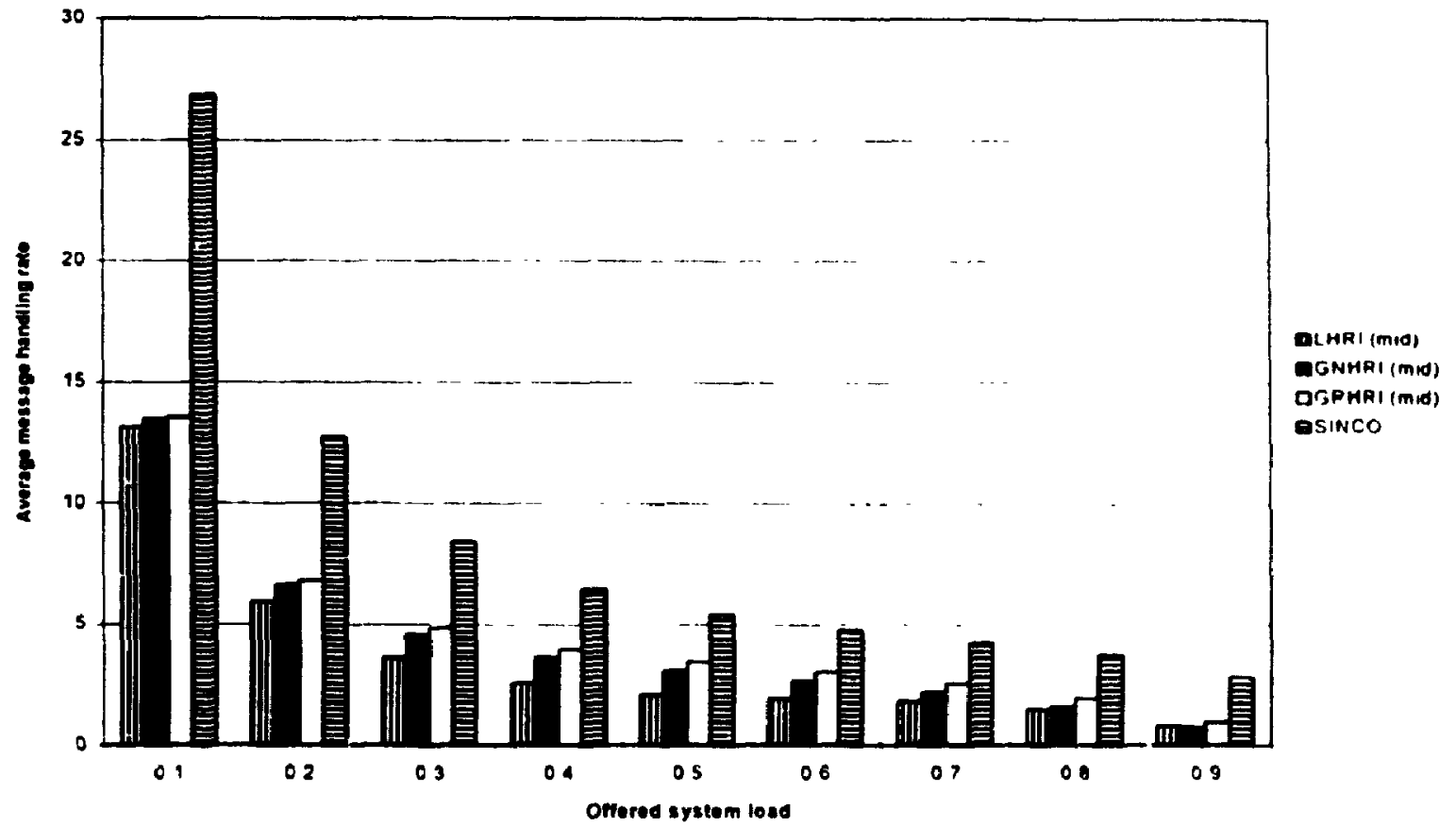

Figure 3.12(b) Average message handling rate at middle level $\left(N=32, B=4, C_{a}=C_{3}=1, i\right.$ is varied tor vary offered system load $\mu=1$ ) 


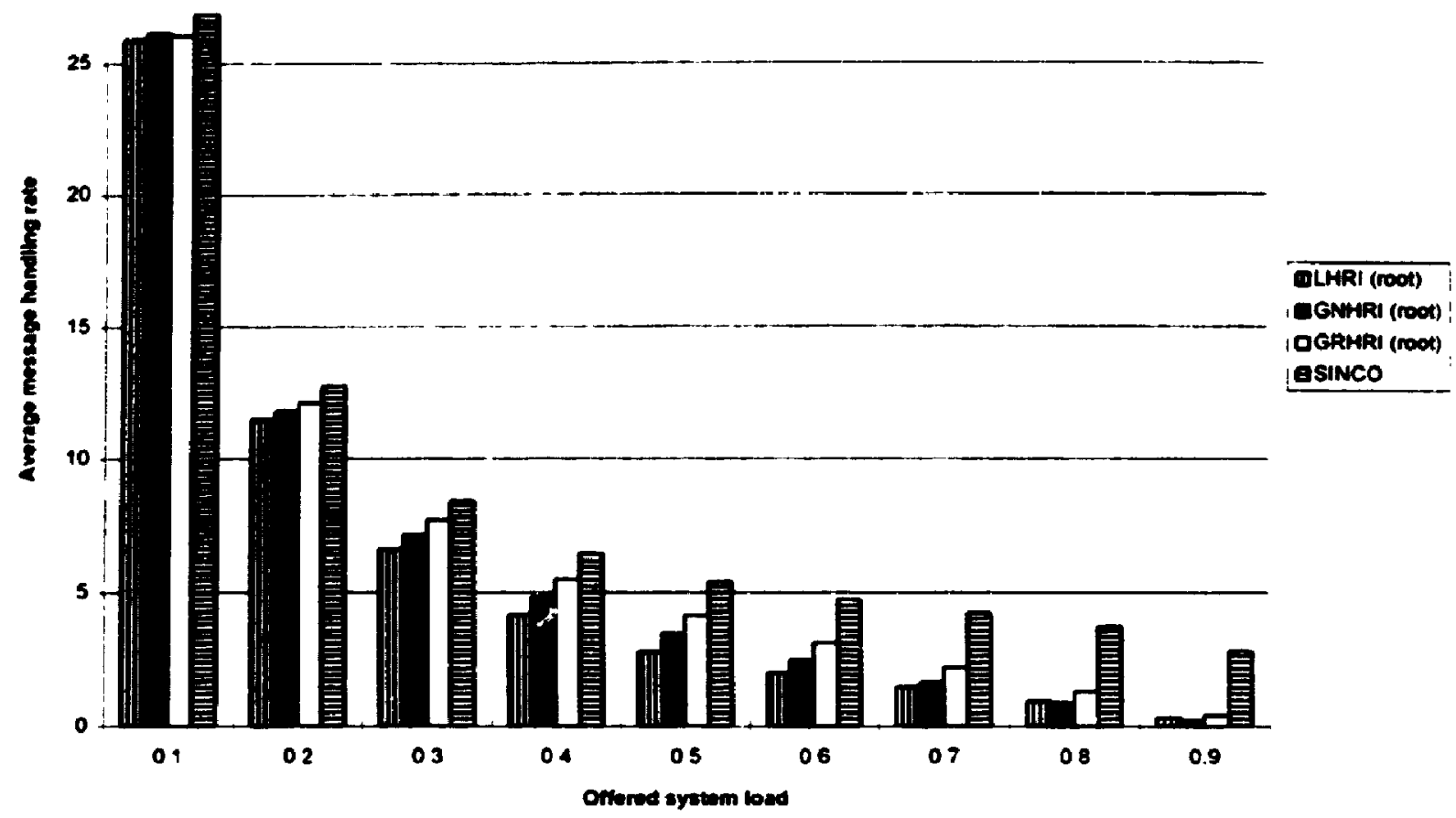

Figure 3.12(c) Average message handling rate at root level $\left(N=32 . B=4, C_{a}=C_{s}=1, \lambda\right.$ is varied to vary offered system load $\mu=1$ )

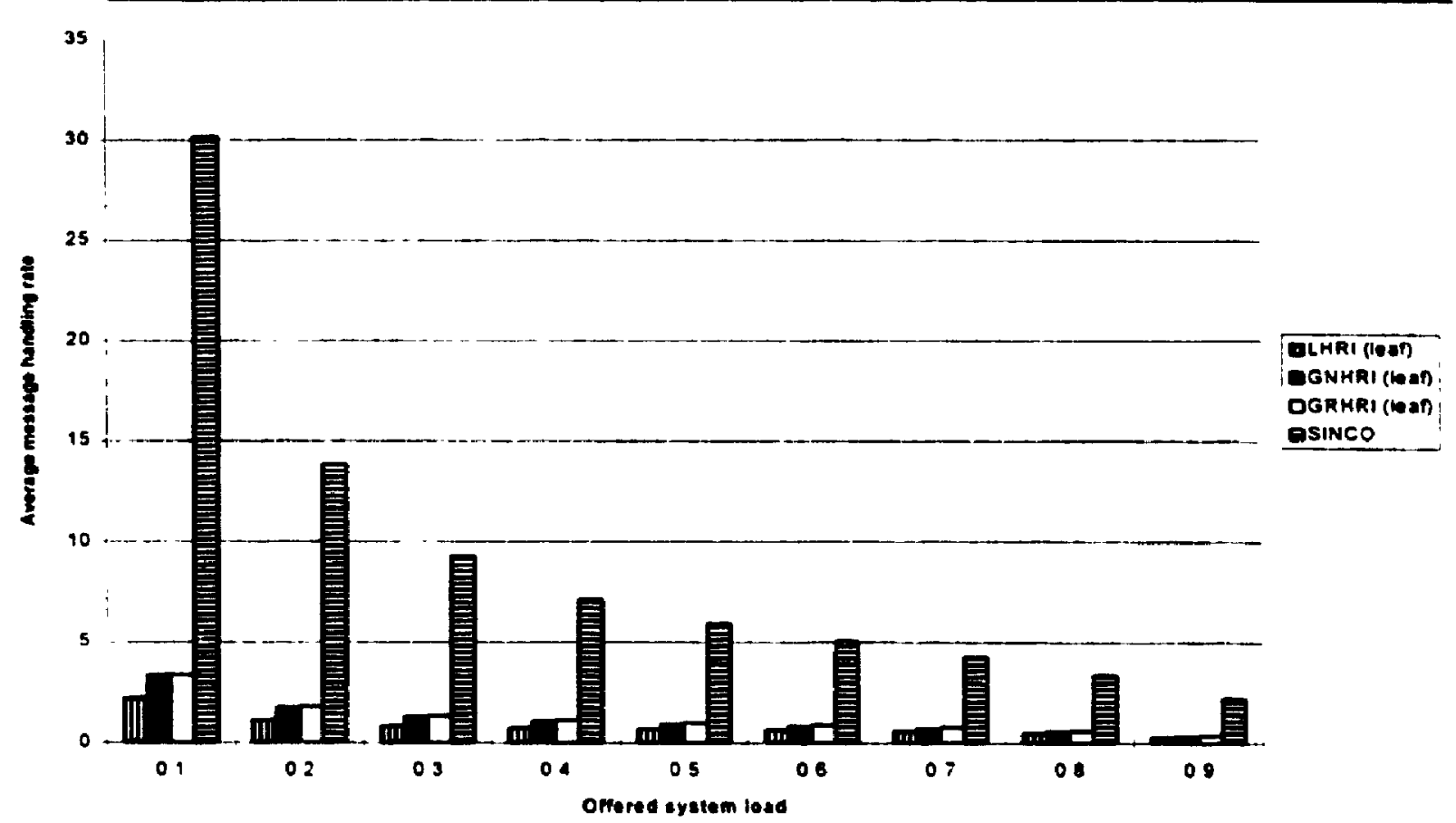

Figure 3.13(a) Average message handling rate at leaf level $N=32, B=4, C_{1}=C_{s}=4, \lambda$ is varied to vary offered system load. $\mu=1$ ) 
35 -

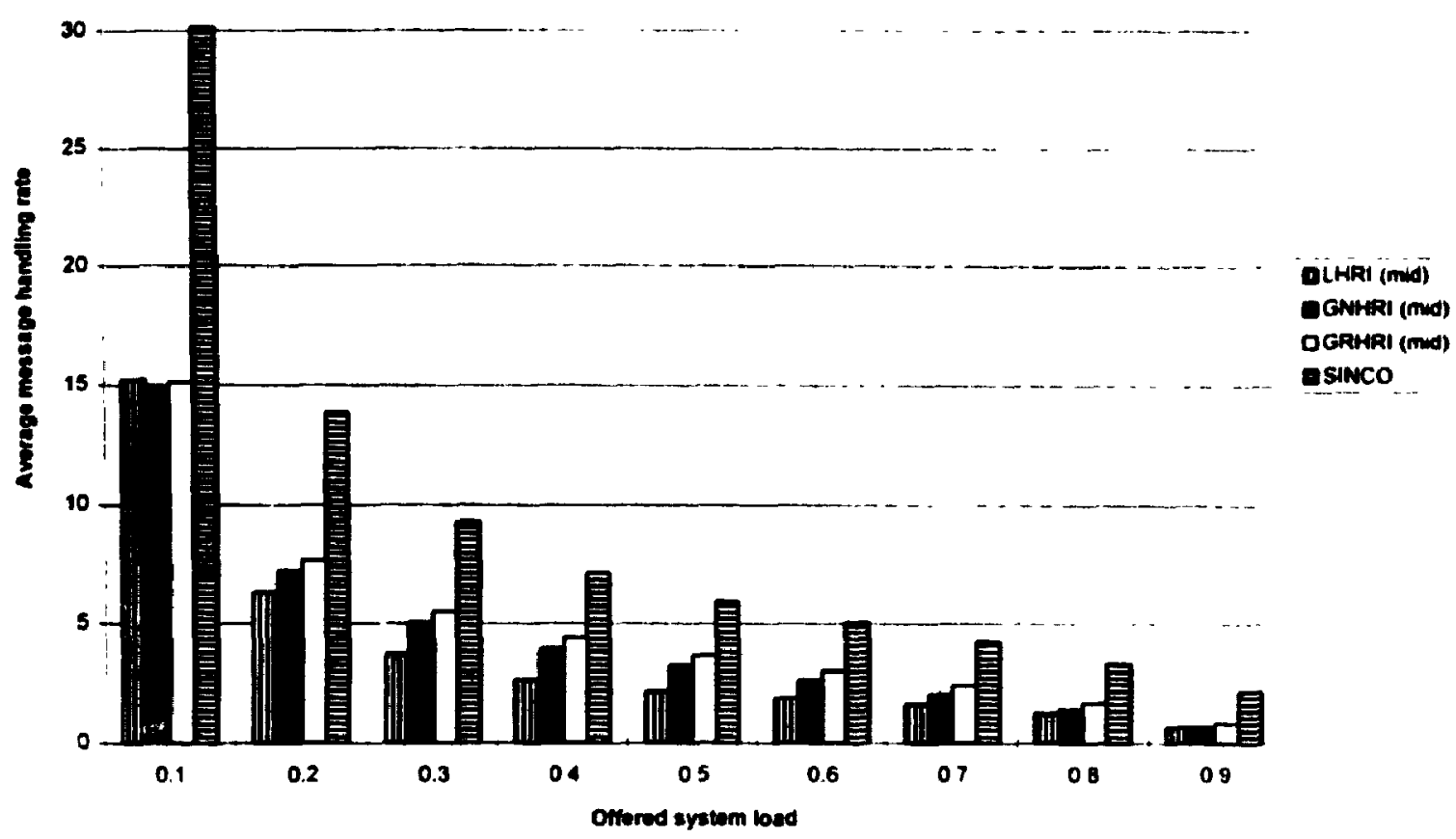

Figure 3.13(b) Average message handling at middle level $\left(N=32 . C_{a}=C_{3}=4, \lambda\right.$ is varied to vary offered s) jtem load $\mu=1, B=4$ )

35 .

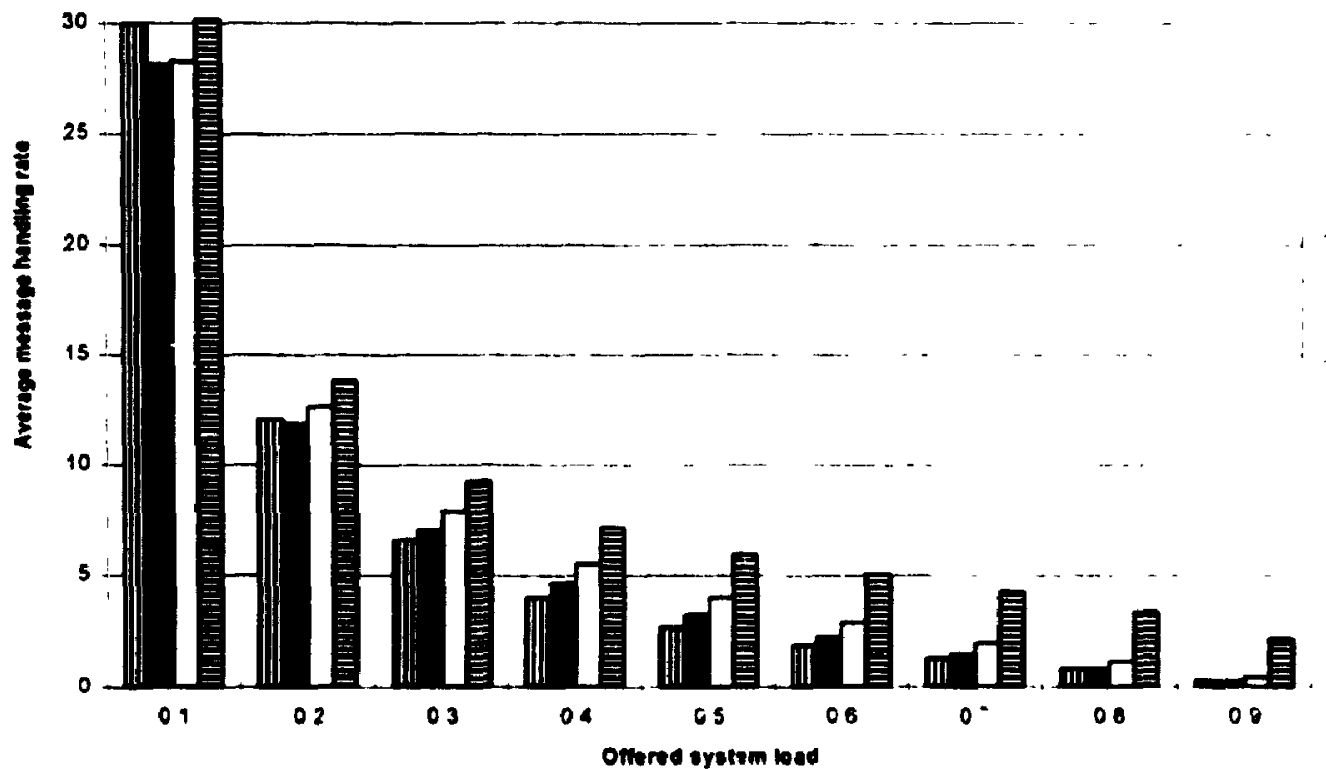

Figure 3.13(c) Average message handling rate at root level $N=32, C_{2}=C_{1}=4, \lambda$ is varied to vary offered system load $\mu=1, B=4$ ) 


\subsection{Summary}

This section summarizes the results presented in this chapter. We have seen in Section 3.4 the performance of six load sharing policies: the sender-initiated (SI), receiver-initiated (RI), periodic symmetrically-initiated (PSI), local hierarchical (LHRI), stable symmetrically-initiated adaptive (SSYM) and single coordinator policies (SINCO). The performance of the two variations of the local hierarchical policy is presented in Section 3.6.

The following are the major observations from the results presented in Section 3.4. LHRI and SSYM are the best alternative to SINCO, which may cause faulttolerance and scalability problems. These three policies yield significant performance improvement over SI, RI, and PSI at high system loads and at high variance in service and inter-arrival times or both. LHRI, SSYM and SINCO are relatively less sensitive to inter-arrival time and service time coefficients of variation (CVs). The experiments show that $\mathrm{SI}$ is more sensitive to service time $\mathrm{CV}$ and $\mathrm{RI}$ is more sensitive to inter-arrival time $\mathrm{CV}$. The performance sensitivity of SI and RI to inter-arrival time and service time CVs match that shown in the literature [Dan95a].

We have also scen that $\mathrm{RI}$ is - Jre sensitive to the probe limit than SI when there is high variation in inter-arrival times and service times. On the other hand, SSYM is not sensitive to the probe limit and thus scalable to large distributed systems. The performance sensitivity of SSYM to probe limit is similar to that given in [Shi90]. 
PSI performs much worse than SSYM at high system loads and at high variance in service and inter-arrival times even though both are symmetrically-initiated policies. This is because the timer period that is used to trigger the location policy, and the small probe limit deter the load sharing activity that should have been generated by senders and receivers.

LHRI outperforms SI and RI substantially at moderate to high loads and at high CVs. This demonstrates that hierarchical organization is effective in reducing the impact of high variation in service times and inter-arrival times and in diminishing the system load imbalance. The performance difference between LHRI and SSYM is marginal (and even indistinguishable). However, the inherent structure of LHRI is more suitable for a distributed system which consists of several LAN clusters.

We have also seen the performance of the two variations of LHR, namely, the global non-recursive (GNHR) and global recursive (GRHRI) hierarchical policies. The results show that GRHRI further improves the performance of the local hierarchical policy by using a global information scheme and recursive search (back tracking). However, it should be noted that when using the global scheme, the advantage of local load sharing is eliminated. Thus, it may not be suitable for a distributed system consisting of several LANs clusters in which the inter-clusters communication cost is high. The performance of GNHRI and LHRI are very close. When the branching factor is small $(B=2)$, LHRI actually yields better performance than GNHRI. The performaince of the three hierarchica: policies improves as the branching factor increases. 
In Chapter 4, we will see the performance of the five load sharing policies and the performance of hierarchical policies in two types of heterogeneous systems. 


\section{CHAPTER IV \\ PERFORMANCE OF LOAD SHARING POLICIES IN HETEROGENEOUS SYSTEMS}

\subsection{Introduction}

In Chapter 3, the results of simulation experiments on the six load sharing policies and on the three hierarchical policies have been discussed. This chapter will present the results of simulation experiments on the five load sharing policies. These policies are SI, RI, SSYM, LHRI and SINCO. PSI is not considered in these experiments because SSYM performs much better than PSI in the experiments discussed in the last chapter, despite the fact that both policies are symmetrically-initiated.

As noted in the literature [Mir90], heterogeneous systems can be broadly classified into two types: Type I and Type II. In a Type I system, all nodes in the system are identical, in terms of processing capabilities and speeds, but the job arrival rates may be different at different nodes. In a Type II system, nodes are functionally identical, but they process jobs at different speeds. In addition the nodes may also be subjected to different job arrival rates as in Type I system.

The remainder of this chapter is organized as follows. Section 4.2 describes the workload and system models for heterogeneous systems. The results of simulation experiments on the five load sharing policies for Type I heterogeneous systems are given in Section 4.3. Sections 4.4 shows the performance of the load sharing policies for Type II systems. The experimental results of three hierarchical policies for Type I and Type II 
systems are discussed in Section 4.5 and Section 4.6 respectively. The final section summarizes the results of these simulation experiments.

\subsection{System and Workload Models}

The system model used in this chapter is the same as that used in Chapter 3, except that the nodes are divided into two classes: Class I and Class II. All nodes in the same class are assumed to be identical and subjected to the same arrival rate of jobs. The workload model for each class is represented by four parameters. The job arrival process at each node in class $i$ is characterized by a mean inter-arrival time $1 / \lambda_{i}$ and a coefficient of variation $C_{2}$. Jobs are characterized by CPU service demand on a class i node with a mean of $1 / \mu_{i}$ and a coefficient of variations $\mathrm{C}_{\mathrm{si}}$. The service times and inter-arrival times are generated using a two-stage hyper-exponential model when the coefficient of variations is greater than one [Kob81]. The mean job response time is the chief performance metric used to compare the performance of the load sharing policies.

\subsection{Performance Comparison of Five Load Sharing Policies in Type I Systems}

This section presents the results of the simulation experiments for the Type I system. The default parameters are as follows. Unless otherwise noted, the default values are assumed in the experiments in this section and subsections. The distributed system consists of $\mathrm{N}=32$ nodes that are connected by a $10 \mathrm{Mbits} / \mathrm{second}$ communication network. The value of Class I node $N_{1}$ is 6 and the value of Class II nodes $N_{2}$ is $N-N_{1}=26$. The mean job service rates for both classes is 1 . The standard message, such as the search message 
and the state update message, is 16 bytes. The CPU overhead to send/receive standard message $T_{m s g}$ or to send/receive probe $T_{\text {probe }}$ is 0.003 time units and to transfer a job $T_{j x}$ is 0.02 time units. Job transfer communication overhead is uniformly distributed $\mathrm{L}_{\mathrm{jx}}=0.009$ and $U_{j x}=0.011$ time units. The upper threshold $T_{h}$ and the lower threshold $T_{1}$ are 1 for SINCO and LHRI for both classes. The transfer threshold $T_{m}$ is 2 for both SI and RI. The threshold $T_{s}$ of the sender-initiated policy and the receiver-initiated policy is 2 and 1 respectively. The threshold T for SSYM is 1 . The thresholds used by a policy are the same among all nodes in the system. The probe limit $P_{1}$ for SSYM. SI, and RI is 3 for all classes. The branching factor (B) for LHRI is fixed at 4 for both classes. The load distribution reinitiation periods, $T_{\text {wait }}$ and $T_{\text {false, }}$, for "no-sender" and "false-sender" are 1 and 0.2 respectively.

Batch strategy has been used to compute confidence intervals (at least 30 batch runs and 30,000 jobs per batch run were used for the results reported here). When the CVs equal 1 , this strategy produced $95 \%$ confidence intervals that were less than $1 \%$ of mean response times when system utilization is low to moderate and less than $5 \%$ at times of high system utilization. When the $\mathrm{CV}$ is set to four and at high utilization ( $80 \%$ or above), the confidence interval is less than $10 \%$ of the mean response time and less than $5 \%$ for low to moderate system utilization. 


\subsubsection{Mean Response Time as a Function of System Load}

Figure 4.1 shows the mean response times of five load sharing policies as a function of offered system load. There are $\mathrm{N}=32$ nodes in the system and the nodes are divided into two classes: Class 1 and Class II. Class $I$ consists of $N_{1}=6$ nodes and Class II consists of $N_{2}=32-N_{1}$ nodes. The offered system load at each class $i$ is given by $\lambda_{i} / \mu_{i}$. As $\mu_{i}=1$, the offered system load is equal to the arrival rate $\lambda_{i}$, where $i=1,2$. The $\lambda_{1}$ is fixed at $90 \%$ and $\lambda_{2}$ is varied to see the performance impact of the system load on Class II nodes. Both the inter-arrival time $\mathrm{CV}$ and the service time $\mathrm{CV}$ are fixed at 1 for both classes in Figure 4.1(a).

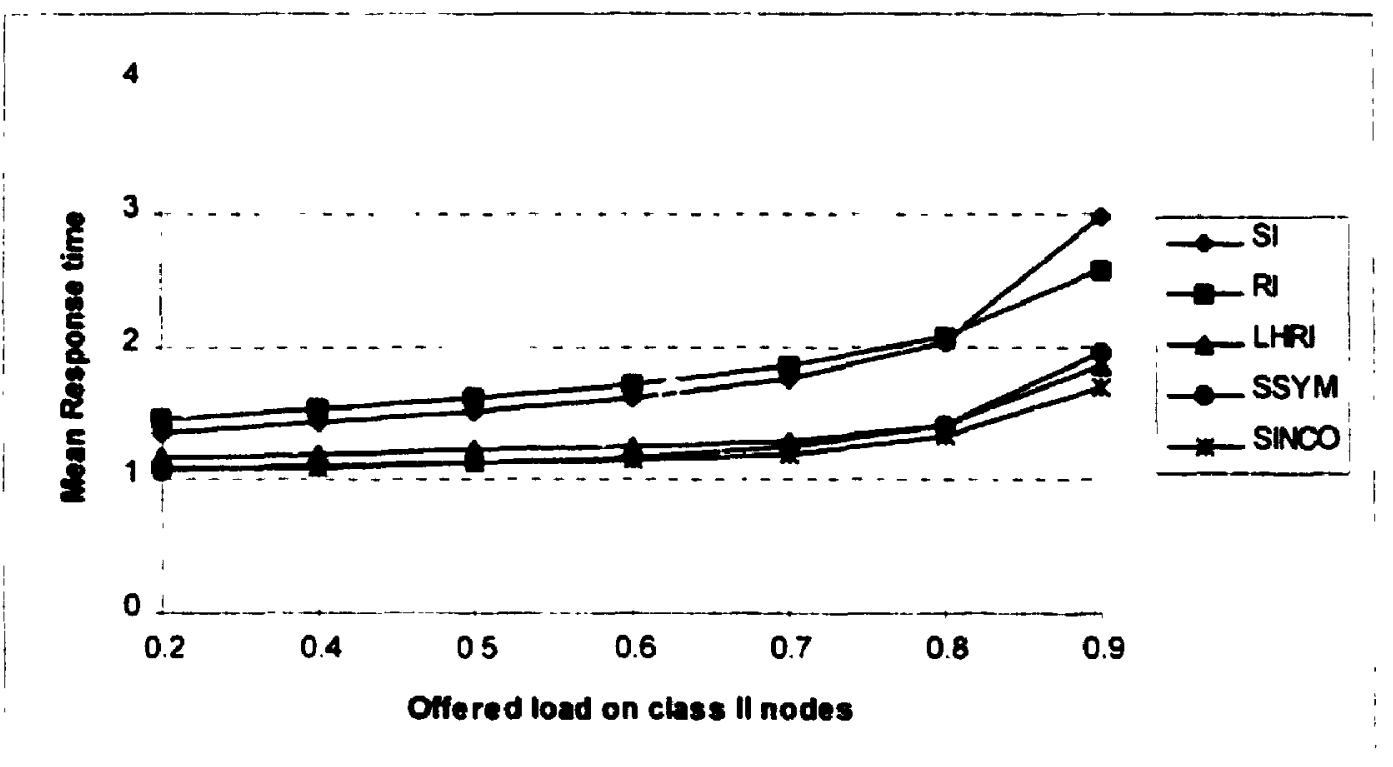

Figure 4.l (a) Mean response time as a function of offered load on Class II nodes. $\left(N=32, N_{1}=6, N_{2}=26\right.$, $\lambda_{1}=0.9, \lambda_{2}$ is varied, $\mu_{1}=1, \mu_{2}=1, T_{1}=T_{n}=1, T=1$ for SSYM, $P_{1}=3$ for SI, RJ SSYM, $T_{m}=2$ for SI and RJ, $T_{3}=1$ for RI. $T_{3}=2$ for Sl, $T_{\text {wat }}=1$ for RI. LHRI and SINCO, $T_{\text {fluse }}=0.2$ for LHRI and SINCO, $B=4$ for LHRI, $C_{a}=C_{s}=1$ ) 
As shown in Figure 4.1(a). for the whole range of loads examined. LHRI. SSYM and SזJCO perform substantially better than SI and RI. At low loads on class 11 nodes. SSYM, SINCO and LHRI exhibit similar performance. At such loads, SSYM has an edge over SINCO and LHRI. There are many receivers but fewer senders in the lightly loaded system; therefore a sender can easily find a suitable receiver for transferring a new extra job. The new job is transferred almost immediately (i.e., after finishing probing). On the other hand, in LHRI and SINCO that job may wait for a longer period before being transferred. This is because receiver-initiated job requests to the coordinator(s) are initiated at the job completion and subsequent initiations are at a rate of $1 / T_{\text {wait }}$ if there is no sender folind during the first initiation. The SSYM has both receiver-initiated and sender-initiated components, but LHRI and SINCO only has a receiver-initiated cumponent. Thus, SSYM transfers jobs immediately from senders to receivers through sender-initiated transfers that increase its performance at low loads on Class II notes SINCO has complete system state information but the job zrival rate at Class II is so low that the receiver-initiated transfers encounter more delays than the sender-initiated transfers of SSYM. Therefore, the performance of SINCO and LHRI deteriorates at low loads on Class II nodes. Note that LHRI incurs more overhead at each search for sender than SINCO, so LHRI yields the highest mean response time among these three policies. The fact that the sender-initiated transfers provide an immediate transfer of new jobs also accounts for why the performance of $\mathrm{RI}$ is worse than that of $\mathrm{SI}$ at low loads. 

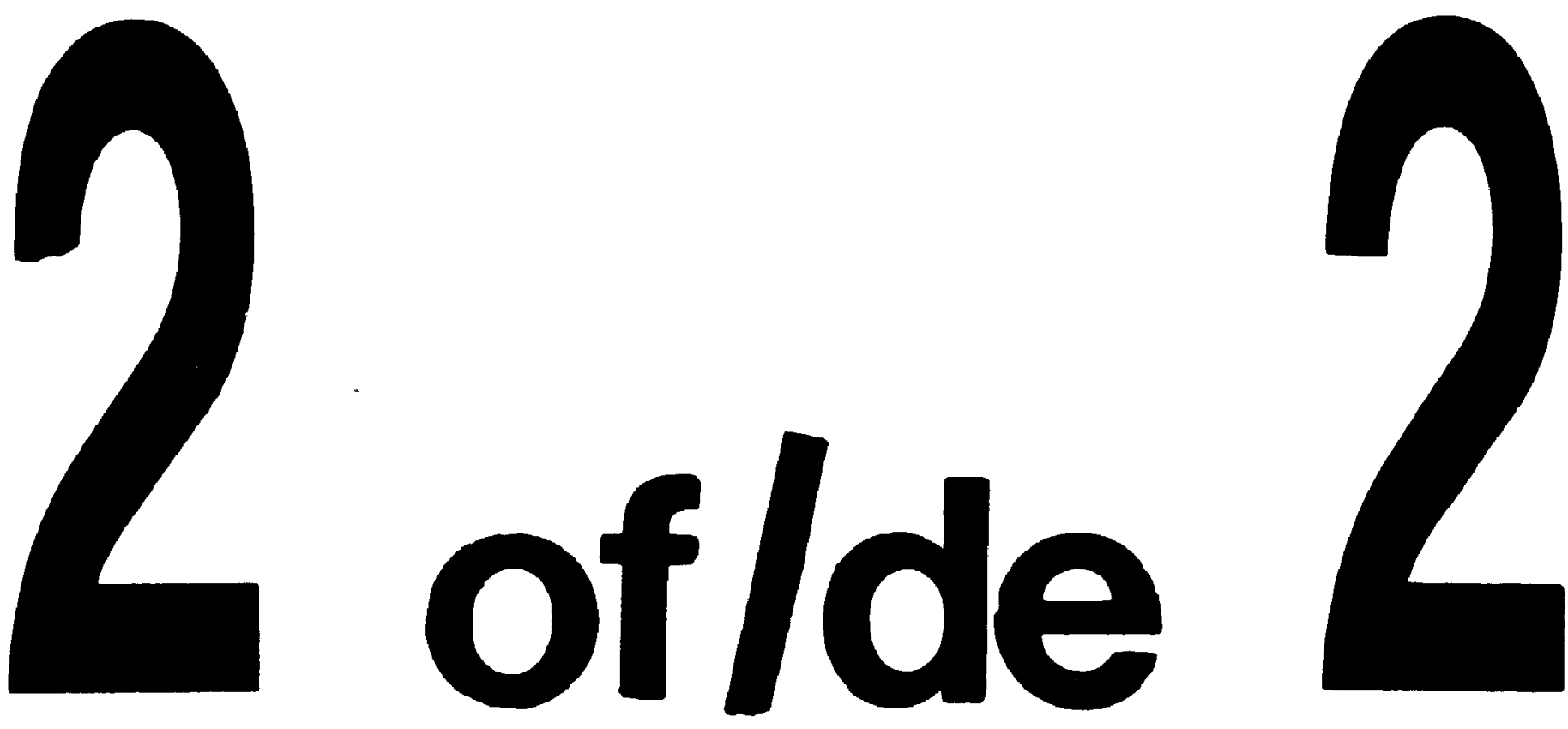

PM-1 31/2"X4" PHOTOGRAPHIC MICROCOPY TARGET NBS 1010a ANSI/ISO *2 EQUIVALENT

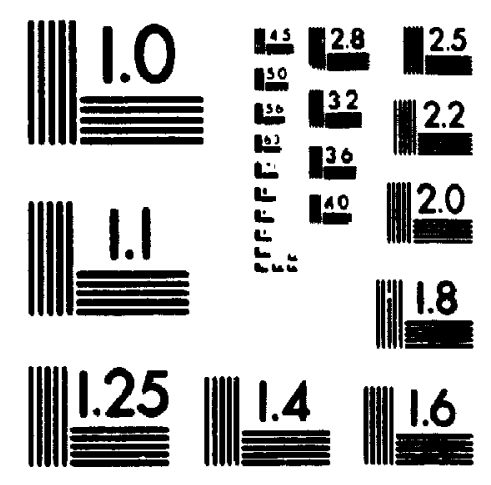

PAECISIONSM RESOLUTION TARIGETS 
At high loads on Class II nodes. SINCO shows marginally better performance than SSYM. This is because it is more difficult for a sender to locate a receiver at high system loads, so the SSYM cannot take advantage of the sender-initiated transfers. Moreover. each fruitless probe for a receiver further increases the burden on the sender node. Since LHRI incurs more CPU overhead than SINCO and in LHRI job transfers encounter longer time delays than those encounter in SINCO, LHRI shows no performance improvement over SSYM at high loads on Class II nodes. LHRI incurs more CPU overhead because more messages are required for each job request (which may flow through several clusters before a sender is located). The increased number of messages for each request implies that the time delays before a request of transferring a job is sert to a sender is longer than that in SINCO.

Figure 4.1(b) shows the mean response time, as a function of an offered system load, for the : iter-arrival time and the service time CVs fixed at 4 . When the loads of class II nodes are low, SINCO cannot outperform SSYM. This follows the same explanation as above, that sender-initiated activity dominates at low loads. Nevertheless, the performance difference among SINCO, SSYM and LHRI is very small and even indistinguishable at low: loads. However, at high loads on Class II nodes, the performance of SINCO is marginally better than that of SSYM and LHRI. The performance of LHRI and SSYM is also similar at high loads on Class II nodes. SINCO, SSYM and LHRI outperforms SI and RI substantially for the whole range of loads on Class II nodes. SI performs marginally better than RI at low loads on Class II nodes whereas at high loads on Class II nodes SI degrades 
rapidly. This performance pattern of SI and RI is the same as that in the homogeneous system.

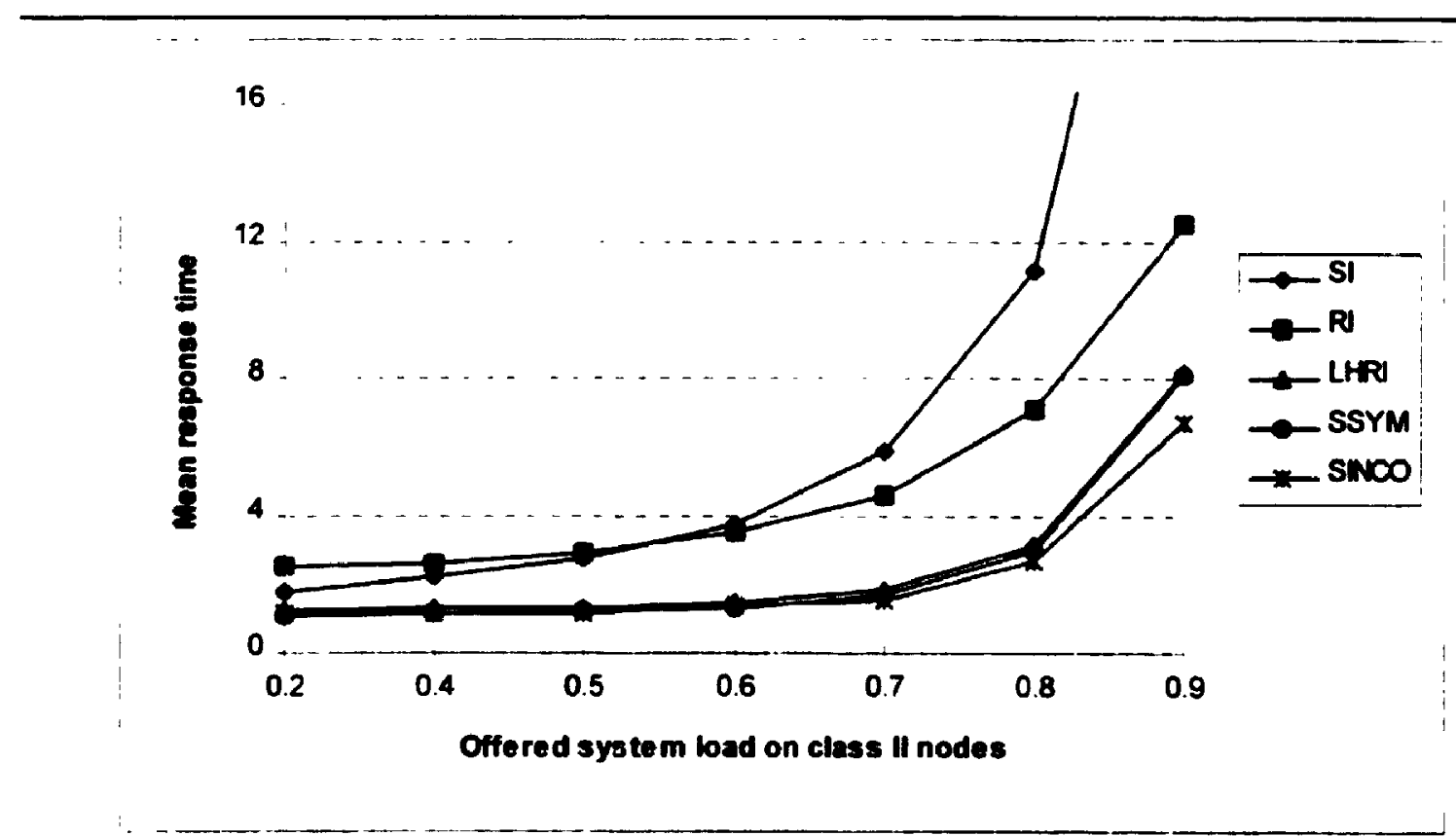

Figure 4.1(b) Mean Response time as a function of offered system load on Class II nodes $\left(N=32, N_{1}=6 . N_{2}=26\right.$. $T_{1}=T_{h}=1, T=1$ for $S S Y M . P_{1}=3$ for $S I$ and $R I, T_{\text {wat }}=1$ for $R I$. LHRI and SINCO, $T_{\text {fase }}=0.2 B=4$ for LHRI and SINCO, $C_{2}=C_{s}=4$ ).

The performance difference between LHRI and SSYM is larger in a heterogeneous system than that in a homogeneous system. This is because although the sender-initiated probes of SSYM sometimes are fruitless at high loads on class II nodes, the probability for a sender to locate a receiver at high loads in such a system is larger than that for a sender to locate a receiver at high loads in a homogeneous system. There are still some nodes subjected to a lower rates of arrivals. Thus, SSYM can still take advantage of immediate new job transfers initiated by senders. On the other hand, LHRI, which has a longer time delays in initiating job transfers and incurs more CPU overhead than SNNCO, is more 
difficult to outperform SSYM at high system loads on Class II nodes. These factors account for the larger performance difference in heterogeneous system.

\subsubsection{Sensitivity to the Inter-arrival Time Coefficient of Variation}

In this experiment, the service time $\mathrm{CV}$ for both classes is fixed at 1 . The interarrival time coefficient of the variation $\mathrm{C}_{a i}$ is set to the same value for each class $i=1.2$. Since the value of $C_{2 j}$ for both classes is equal, the symbol $C_{a}$ is used to represent the common value of the inter-arrival time coefficient of variation for both classes. This representation will also be used whenever a parameter for both classes is equal. The value of $N_{1}$ and $N_{2}$ is 6 and 26 respectively. The arrival rate for Class I and Class II node is fixed at 0.1 and 0.9 respectively. The default values are used for all other parameters. The results are shown in Figure 4.2.

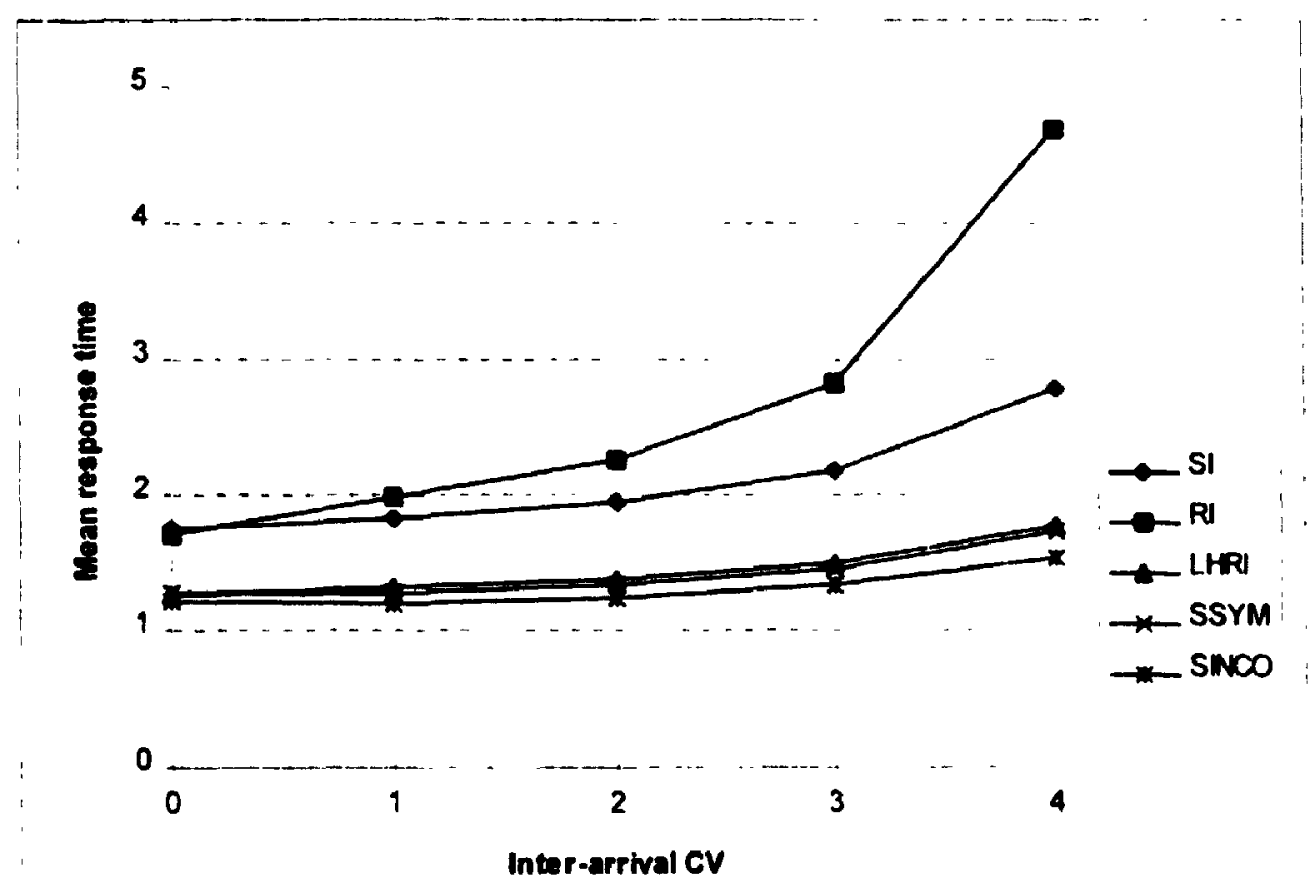


Figure 4.2 Performance sensitivity to inter-arrival $C V N=32, N_{1}=6, N_{2}=26, \lambda_{1}=0.1 \lambda_{2}=0.9 C_{2}$ is varied from 0 to 4 and $C_{4}$ is fixed at 1.)

SI shows less sensitivity to the inter-arrival time coefficient of variation than $\mathrm{RI}$. But both SI and RI show substantially higher sensitivity to $\mathrm{C}_{\mathrm{a}}$ than LHRI SSYM and SINCO. The greater performance sensitivity of RI to high variance in inter-arrival times is because increased $\mathrm{C}_{\mathbf{a}}$ implies a clustered nature of job arrivals into the system. This reduces the number of job transfers initiated by receivers under RI because each node must finish the jobs in a cluster before it initiates any probe. On the other hand, the clustered nature of job arrivals cause senders to initiate more probes in SI. Therefore SI is less sensitive to the variance in inter-arrival times than $R I$.

LHRI is marginally more sensitive to the inter-arrival time CV than SSYM. The mean response time of LHRI increases $40 \%$ while that of SSYM only increases $35 \%$ when the inter-arrival time $\mathrm{CV}$ changes from 0 to 4 . This is because the receiver-initiated load sharing activity under LHRI is reduced at high variance in inter-arrival times, as expleirad for the behavior of basic RI. In contrast the sender-initiated activity of SSYM increases as the CV increases, because SSYM not only triggers receiver-initiated transfers but also triggers sender-initiated transfers. Note that at any CV value the performance of SSYM is marginally better than that of LHRI. This is due to the fact that there are more highly loaded nodes in the system than lightly nodes that again gives ar. edge to sender-initiated activity to transfer more jobs from sender nodes to idle nodes. In this heterogeneous system, there are six nodes subjected to a lower arrival rate of jobs. These idle nodes could 
definitely alleviate the burden on those overloaded nodes through either sender-initiated transfers or receiver-initiated transfers of SSYM.

SINCO shows least sensitivity to the inter-arrival time CV, even though it only has a receiver-initiated component. SINCO utilizes the global picture of the system state, which most of the time is up-to-date, to allocate a job from a sender to a receiver for each receiverinitiated job request. This reduces the impact of fewer receiver-initiated requests at high variance in inter-arrival times.

\subsubsection{Performance Sensitivity to Variance in Service Times}

In this experiment, the inter-arrival time $\mathrm{CV}$ is set to one and the service time coefficient of variation $C_{s}$ varies from 0 to 4 for both classes. The $N_{1}$ and $N_{2}$ are 6 and 26 respectively. The job arrival rate at Class I is 0.1 and that of Class II is 0.9 . Figure 4.3 illustrates the performance sensitivity of the five policies to variance in service times.

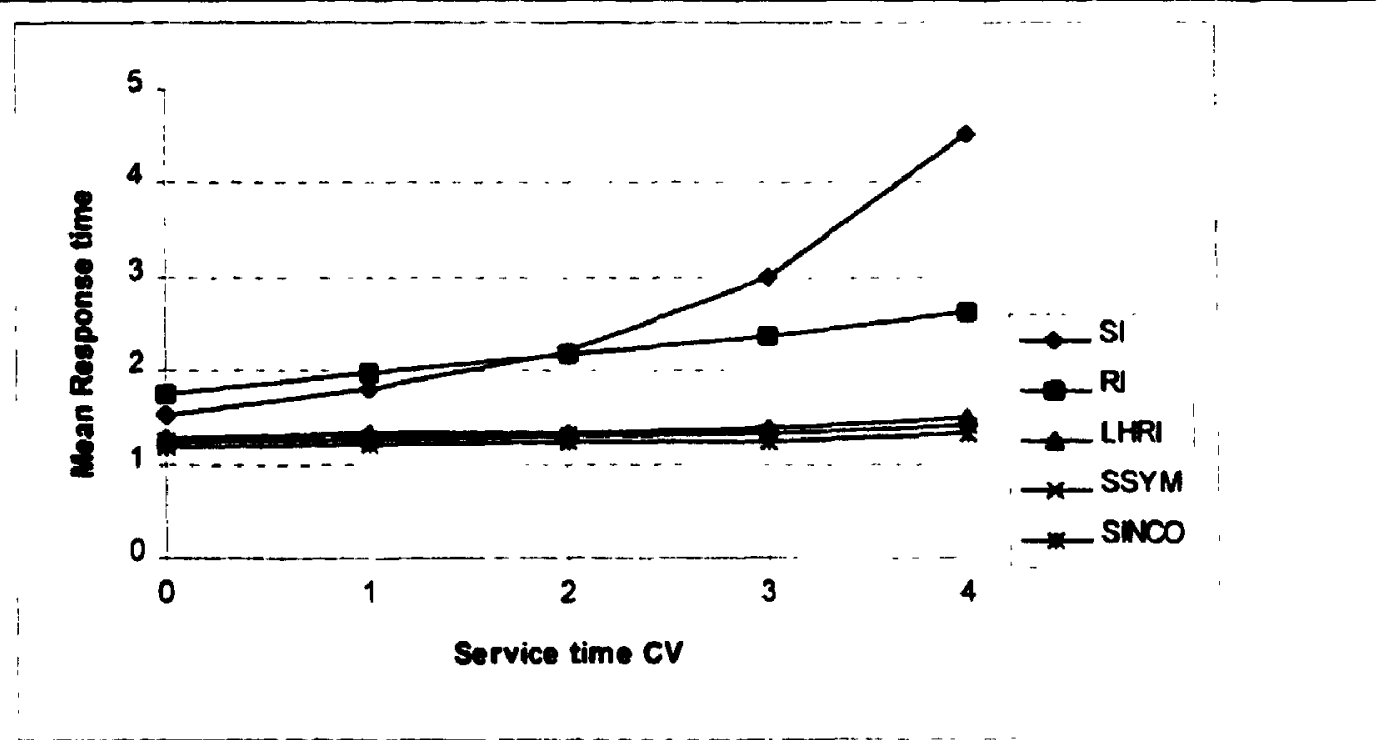

Figure 4.3 Impact of variance in service times $\left(N=32, N_{1}=6, N_{2}=26, \lambda_{1}=01 \lambda_{2}=0.9 C_{3}\right.$, s varied from 0 to 4 and $C_{2}$ is fixed at 1 .) 
At high variance in service times most of the nodes are monopolized by long jobs, so more sender-initiated probes are generated. However, the increased probes at each node may not result in more job transfers from busy nodes to lightly loaded nodes, since SI may not able to locate the existing lightly loaded nodes in the system through the random probing process. Each fruitless probe places an extra burden on those busy nodes. Therefore, $\mathrm{SI}$ is the most sensitive policy to the service time CV. Unlike the senderinitiated random protes, the receiver-initiated random probes do not consume useful CPU time when there is work to be done. This results in less sensitivity to variance in service times.

Even though receiver-initiated random probes do not impose an extra burden on busy nodes, they are still not very effective in locating sender nodes for receiver nodes, so the performance is still inferior to that of LHRI. SSYM is relatively less sensitive to the service time CV than LHRI. This is because SSYM cumbines the benefits of controlled sender-initiated activities, which reduces fruitless probes and the full benefit of receiverinitiated activities. SSYM accurately locates Class I nodes through the use of state lists within each node. So SSYM can transfer more jobs from highly loaded nodes to idle nodes through sender-initiated transfers as there are six very lightly loaded nodes, which are very likely to be idle, in Class I. In addition, the benefits of receiver-initiated transfers which do not cause extra burden to busy nodes are also augmented in the SSYM policy. On the other hand. LHRI can only obtain the benefits from receiver-initiated activities but not senderinitiated activities. Thus, SSYM is marginally less sensitive to such variance in such a 
heterogeneous system. As expected, SINCO is the least sensitive policy to the variance in service times, as complete state information is given for each search for a sender.

\subsubsection{Sensitivity to the Degree of Heterogeneity}

This section presents the performance sensitivity of the five load sharing policies to the degree of heterogeneity. The distributed system is divided into two classes. The number of Class II nodes, $\mathrm{N}_{2}$, is varied and the number of Class I nodes is $\mathrm{N}_{1}=\mathrm{N}-\mathrm{N}_{2}$. The offered loads on Class I and Class II nodes are fixed at 0.1 and 0.9. (i.e.. $\lambda_{1}=0.1, \lambda_{2}=0.9$ ) As the number of Class II nodes increases the mean response time of all load sharing policies increases. This is due to the fact that as $\mathrm{N}_{2}$ increases so do the number of heavily loaded nodes. Figure 4.4 plots the mean response time as a function of Class II nodes.

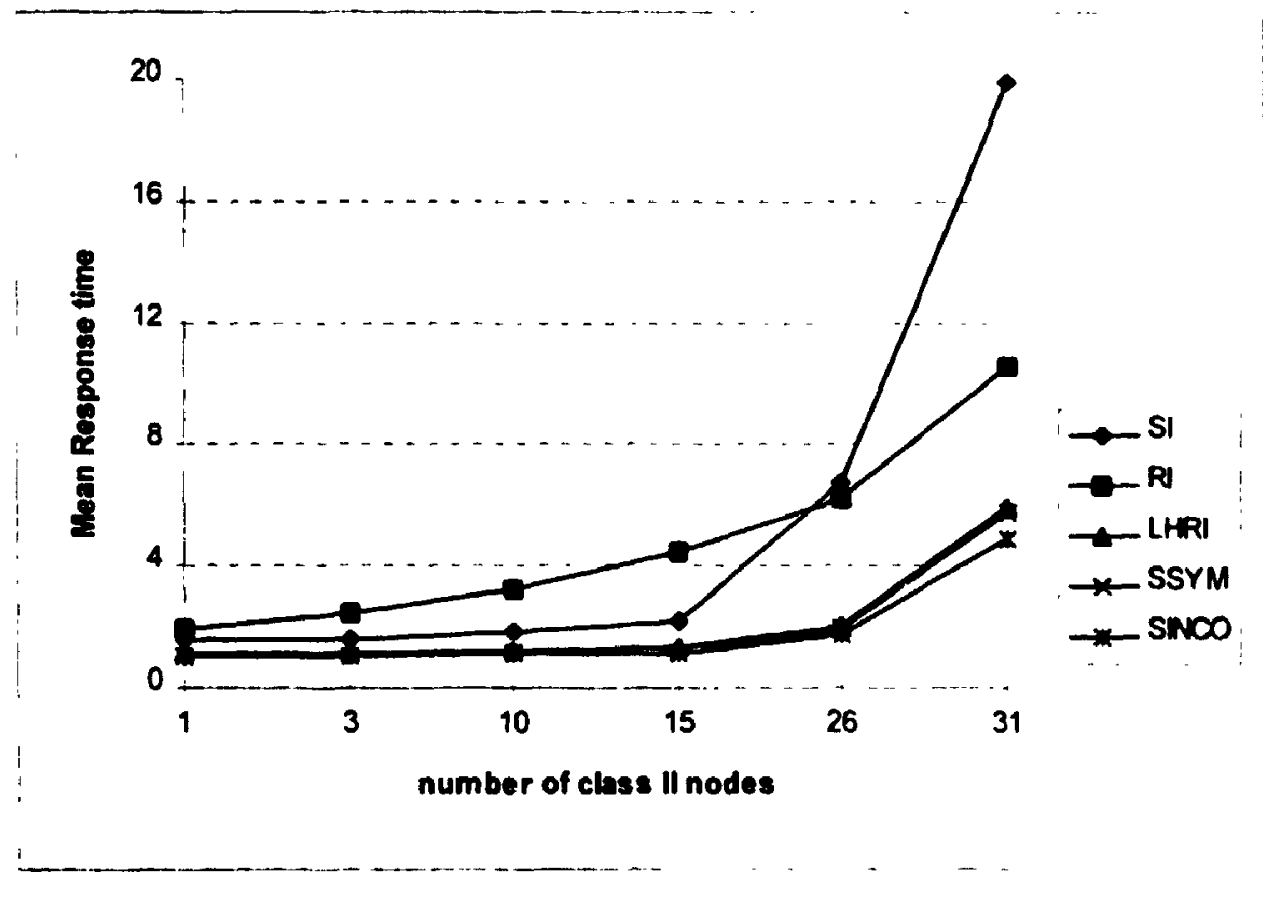

Figure 4.4 Impact of the degree of heterogeneity $\left(N=32, N 2\right.$ is varied and $\left.N_{1}=N-N_{2}, C_{1}=C_{1}=4 \lambda_{1}=0.1 \lambda_{2}=0.9\right)$ 
SI performs better than RI when the number of class II nodes is smaller than the number of class I nodes which are lightly loaded. This is because in this situation the probability of a sender node locating a receiver through random probing is high. On the other hand, as $\mathrm{N} 2$ increases, the probability of a receiver node finding a sender node is high, so RI outperforms SI. The performance of SSYM, LHRI and SINCO is the same except that when the number of Class II nodes is 31 , SINCO yields a marginally better performance. In this case, senders find it relatively more difficult to locate a receiver. The sender-initiated component of SSYM is relatively less active in this system so most job transfers are initiated by receivers. When the number of class II nodes is larger, SSYM becomes like a receiver-initiated policy and its performance now depends mainly on how effective it locates a sender with the state lists at each node. The results shows that SSYM is no more effective than LHRI in locating a sender. In SINCO, a single node maintains the state of all nodes so it is more effective in finding a sender for receivers than the other two policies. However, when the number of Class II nodes is small, SINCO exhibits same performance as that of SSYM because the sender-initiated component of SSYM allows job transfers to be initiated almost immediately upon the arrival of new jobs. In addition, because the job arrival rate of Class I nodes is so low that the new jobs wait longer to be transferred than that to be transferred if SSYM is used. Since SINCO cannot outperform SSYM when number of Class II nodes is small, LHRI shows no improvement over SSYM for all the values of $\mathrm{N}_{2}$ tested (it has been noted before LHRI cannot do better than SINCO). 


\subsection{Performance of Five Load Sharing Policies in Type II Systems}

This section presents the performance of the five load sharing policies in a Type II heterogeneous system. In a Type II heterogeneous system. nodes are functionally identical but have different processing rates and may possibly be subjected to different job arrival rates. As in the previous sections, there are $\mathrm{N}=\mathbf{3 2}$ nodes in the system and the nodes are divided into two classes: Class I and Class II. The system and workload models used are the same as those described in Section 4.2.

\subsubsection{Performance as a Function of System Load}

Figure 4.5 shows the mean response time as a function of offered system load. The nodes in the system are equally divided into two classes (i.e., $N_{1}=16, N_{2}=16$ ). The processing rate of Class I nodes is $\mu_{1}=0.5$ and the processing rate of Class II nodes is $\mu_{2}=1$. In order to maintain the same offered system load $(\lambda / \mu)$ for both classes, the job arrival rate of Class I is kept at half of that for Class II because Class II nodes are twice as fast (i.e. $\lambda_{1}=\lambda_{2} / 2$ ). The thresholds $T_{m 1}$ and $T_{m 2}$ are 2 and 3 respectively for $S I$ and RI. The threshold $T_{s}$ is 1 for both classes in RI policies. In SI, $T_{s 1}$ and $T_{s 2}$ are equal to $T_{m 1}$ and $T_{m 2}$ respectively. The threshold $T_{1}$ and the threshold $T_{h}$ are both 1 for both classes of nodes for LHRI. The threshold T for SSYM is 1 . The inter-arrival time and service time coefficient of variations are both 4 for both classes. The average communication cost for each job transfer is $1 \%$ of the mean service time at Class II nodes and it is uniformly 
distributed. The branching factor $B$ for $L H R I$ is fixed at 4 . The re-initiation periods $T_{\text {wait }}$ and $T_{\text {false }}$ are 1 and 0.2 , respectively.

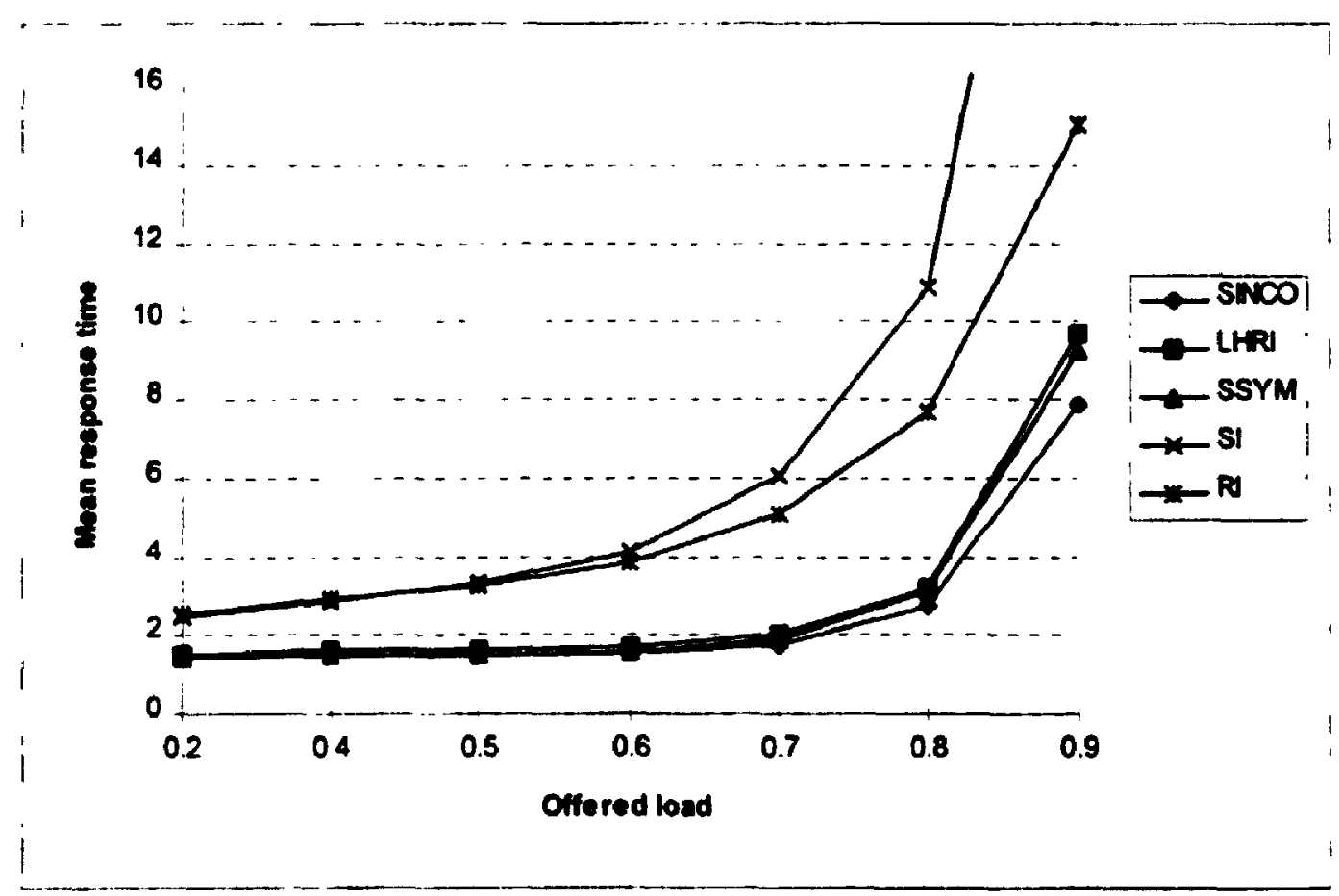

Figure 4.5 Performance sensitivity to offered system load $N=32, N_{1}=N_{2}=16, \lambda_{1}=\lambda_{2} / 2, \lambda_{2}$ is varied $1 \mu_{1}=0.5$ $\mu_{2}=1, C_{a}=C,=4 B=4$ for LHRI $T_{m 1}=T_{11}=2, T_{m 2}=T_{s 2}=3$ for $S 1 ; T_{m 1}=2 T_{m 2}=3, T_{31}=T_{32}=1$ for $R I, T_{w a t}=1$ for LHRI and RI. $T_{\text {fuse }}=0.2$ for false sender in LHR)

SINCO yields the lowest mean response time among the five load sharing policies.

SI provides marginally better performance than RI at low to moderate loads but substantially worse performance than $\mathrm{RI}$ at very high loads. This is because at high system loads it is more difficult for a sender to find a receiver, particularly for slower nodes in Class I. The performance difference between SSYM and LHRI is indistinguishable. In other words, they perform equally well at such system. However, the performance of SINCO, SSYM and LHRI shows substantial performance improvement over the senderinitiated and the receiver-initiated policies. 


\subsubsection{Impact of the Degree of Heterogeneity}

In this experiment, the nodes are again divided into two classes. The job arrival rate at Class I nodes and job arrival rate at Class II nodes are fixed at 0.4 and 0.8 respectively. The processing rate of Class I and Class II nodes is the same as that noted in Section 4.4.1. Thus, nodes in both classes are subjected to the same offered load. The number of Class I nodes (slower nodes) $N_{1}$ is varied and the number of class II nodes $N_{2}$ is given by $32-N_{1}$. All other parameter values are set as in Section 4.4.1.

Figure 4.6 shows the mean response time versus the number of Class I nodes. When we move from left to right in this figure. the mean response time of the five policies increases because the number of slower nodes increases. SI is the most sensitive to the degree of heterogeneity. This is because a sender has a higher probability to locate a receiver when there are fewer Class I nodes (slower nodes) than when there are more Class I nodes. SINCO shows the least performance sensitivity to the degree of heterogeneity. This is due to the fact that it has the complete state information for the whole system so a receiver is very likely to acquire a job from a sender by consulting a single node. Both SSYM and LHRI yield similar performance for all values of $\mathrm{N}_{1}$ examined. Unlike SINCO, LHRI cannot outperform SSYM even when there are more Class I nodes that causes SSYM to become like a receiver-initiated policy. This is because the time delays associated with job requests initiated by receivers in LHRI is longer than that in SINCO. Nevertheless, SINCO, SSYM and LHRI exhibit less sensitivity to the degree of heterogeneity than SI and 
RI. We will see the performance of the three hierarchical policies in Type I and Type II in Section 4.5 and Section 4.6.

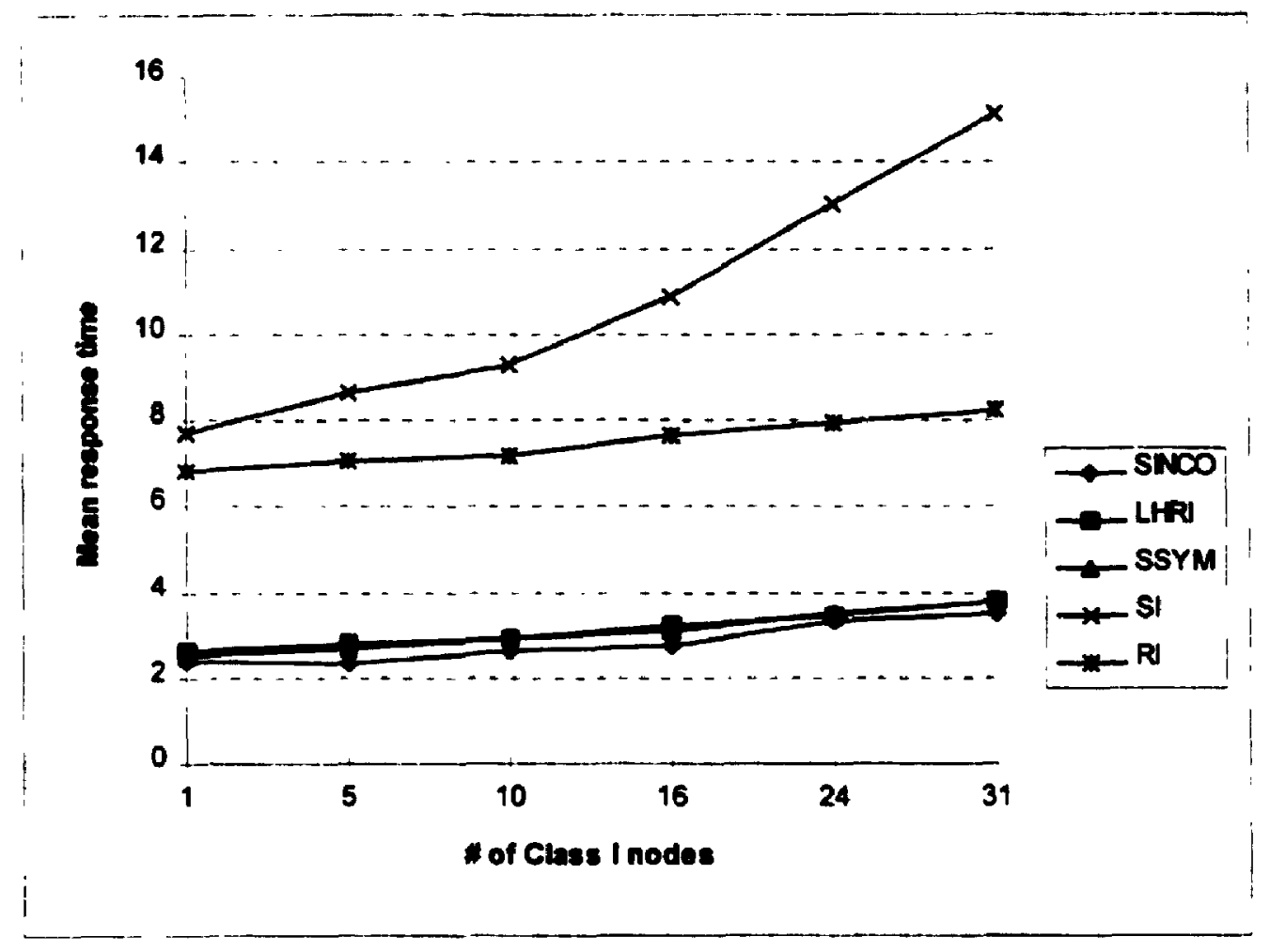

Figure 4.6 Mean response time as a function of \# of class 1 nodes $\left(N=32, N_{1}\right.$ is varied. $N_{2}=N-N_{1}$ $\lambda_{1}=0.4, \lambda_{2}=0.8, \mu_{1}=0.5 \mu_{2}=1, C_{3}=C_{3}=4, B$ is fixed at 4 for LHRI) 


\subsection{Performance of Hierarchical Policies in Type I Heterogeneous Systems}

As described in Chapter 3, the global non-recursive policy differs from the local policy in the state information maintained at the hierarchical. The two states for each node are the sender state and the non-sender state (OK or RECEIVER). These two states are represented by 1 and 0 respectively. In addition to the state information maintained in the hierarchy, the global recursive policy also incorporates back tracking in searching for a sender.

The system and workload models used in this section are the same as those described in Section 4.1. The following are the default parameters. The branching factor (B) of these three hierarchical policies is fixed at 4 for all experiments. The thresholds $T_{h}$ and $T_{1}$ are both fixed at 1 . The load distribution re-initiation period $T_{\text {false }}$ $\left(T_{\text {wail }}\right)$ for the "false-sender" (no job) scenario is fixed at $0.2(1)$. The nodes in the system are divided into two classes: Class I and Class II. The number of Class I nodes, $N_{1}$, is 6 and the number of Class II nodes, $N_{2}$, is 26 . The offered system load at each class $\mathrm{i}$ is defined as

$\lambda_{i} / \mu_{i}$. The mean service demand is 1 for both classes.

\subsubsection{Performance as a Function of Offered System Load on Class II Nodes}

Figure 4.7(a) shows mean response time as a function of offered system load on

Class II for both CVs fixed at 1 for both classes. The $\lambda_{2}$ is varied to vary the system load on Class II nodes. We can see that global policies provide marginally better performance than 
LHRI over the whole range of offered system loads examined. However the performance difference between the global recursive policy (GRHRI) and the local policy (LHRI) is larger than that between the global non-recursive and the local policy particularly at high system loads on Class II nodes. This is because with back tracking, GRHRI has a higher probability of locating a sender than the other policies. Unless the state information maintained in the hierarchy does not reflect any sender in the system (because of communication delays), it is very unlikely at high loads on both classes, that a receiver would not acquire a job from a sender. In the global non-recursive policy, a receiver may need to wait for re-initiation before acquiring a job from a sender, because of the failure of the first initiation due to the "false-sender" scenario. Thus, the time delays before a receiver initiates a transfer may be higher in GNHRI than that in GRHRI.

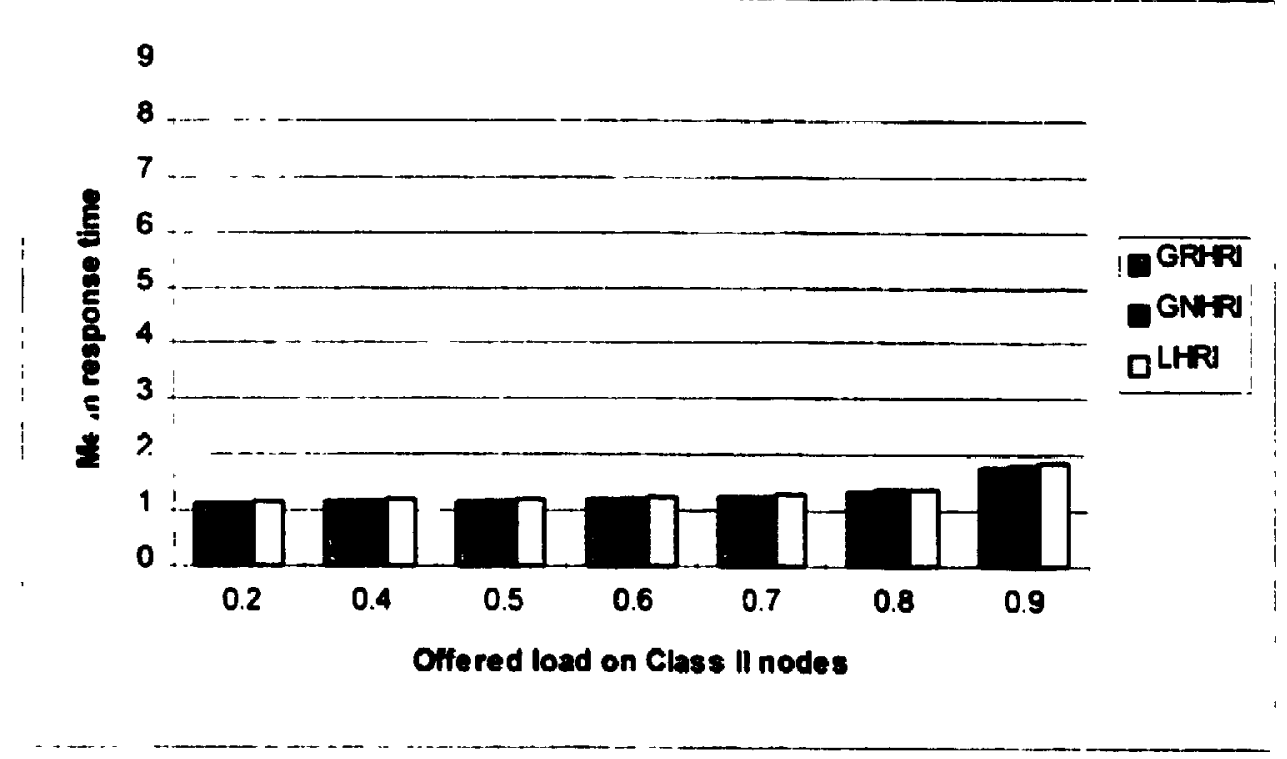

Figure 4.7 (a) Performance as a function of system load on Class II nodes $\mathrm{N}=32, \lambda_{2}$ is varied to vary load. $\lambda_{1}=0.9, \mu=1$ for both classes. $B=4 . T_{1}=T_{h}=1 . C_{s}=C_{1}=1, r_{\text {false }}=0.2 . T_{\text {mail }}=1$, transfer cost is $1 \%$ of $\mu$ ) 
Figure 4.7(b) shows the mean response time as a function of system load on Class II nodes. This figure corresponds to both CVs of 4 for both classes of nodes. The performance difference among the three policies at high system loads on Class II nodes is larger than that when CVs is set at 1 . The GRHRI provides the best performance at high system loads on Class II nodes. The performance of GNHRI is marginally better than that of LHRI at low to moderate loads on Class II nodes. At high system loads on Class II nodes, the time delays associated with acquiring a job from a sender in LHRI are diminished, and these time delays are (usually) longer because LHRI encourages local load sharing. At $90 \%$ of load, LHRI yields marginally lower response time than GNHRI. This is not only because the net effect of longer time delays associated with local sharing is diminished at very such a load, but also due to the benefits of fewer "false sender" cases when local sharing is given higher priority. Nevertheless, the GRHRI still performs better than LHRI and GNHRI at $90 \%$ load due to the use of back tracking searches for senders.

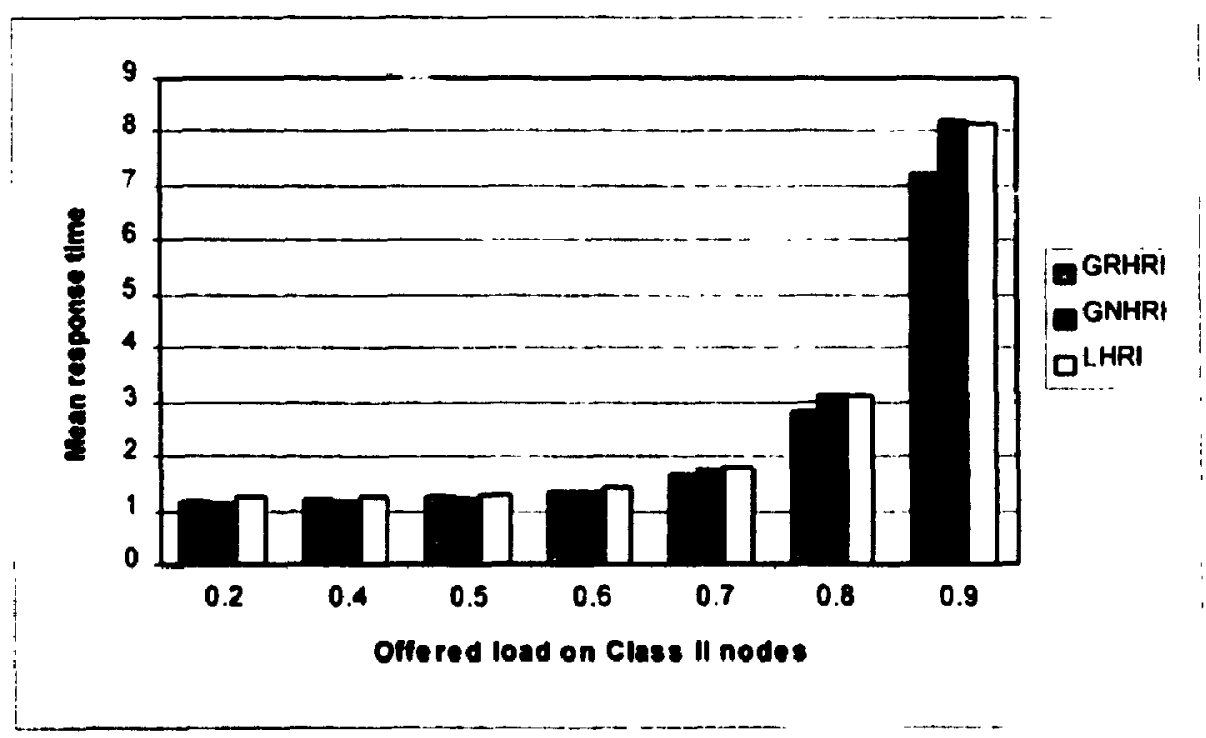

Figure 4.7(b) Performance as a function of Offered load on Class II nodes $N=32, \lambda_{2}$ is varied to vary load, $\lambda_{1}=0.9, \mu=1, B=4, T_{1}=T_{h}=1, C_{1}=C_{1}=4, T_{\text {false }}=0.2, T_{\text {wat }}=1$, transfer cost is $1 \%$ of $\mu$ ) 


\subsubsection{Sensitivity to Variance in Inter-Arrival Times}

This section shows the performance sensitivity to the variance in inter-arrival times. In this experiment, the service time coefficient of variation $\left(\mathrm{C}_{\mathrm{s}}\right)$ is fixed at 1 for both classes of nodes. The branching factor (B) is assumed to be 4 for the three hierarchical policies. The number of Class I nodes is 6 and the number of Class II nodes is 26. The offered load of Class $I$ and Class II nodes is fixed at 0.1 and 0.9 respectively. Figure 4.8 shows the performance sensitivity of the three hierarchical policies to the inter-arrival time CV.

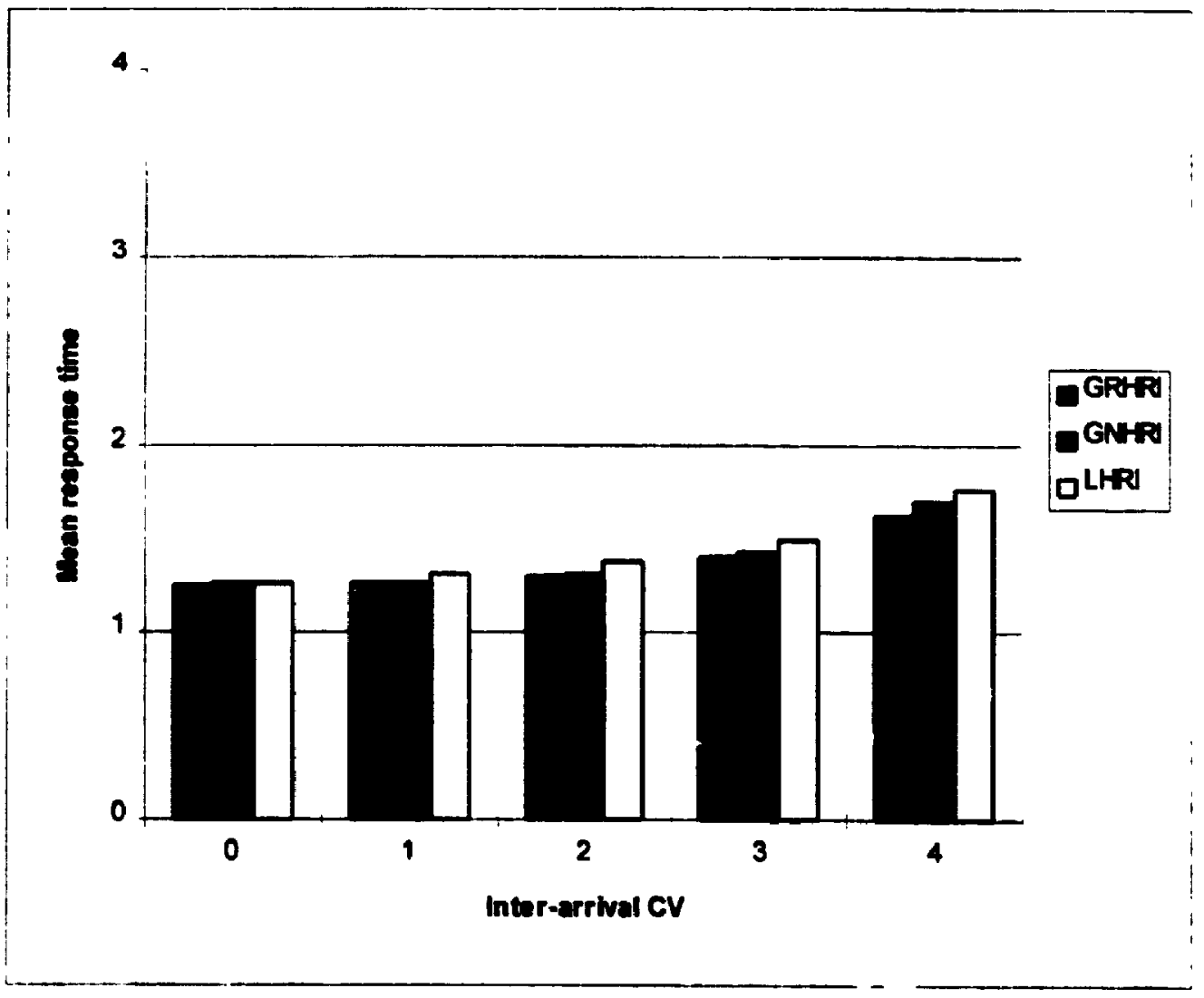

Figure 4.8 Performance sensitivity to inter-arrival $C V\left(N=32, N_{1}=6 N_{2}=26, C_{8}=1, C_{2}\right.$ is varied, $B=4$, $T_{h}=T_{1}=1, \lambda_{1}=0.1, \lambda_{2}=0.9, \mu_{1}=\mu_{2}=1 . T_{\text {mat }}=1, T_{\text {false }}=0.2$ transfer cost $i .1 \%$ of $\mu_{1}$ ) 
There is a small increase in mean response time when the inter-arrival time coefficient of variation $\left(C_{2}\right)$ changes from 0 to 3 . However, when the $C_{2}$ is increased from 3 to 4 , the mean response time of three policies increases to a greater extent. GRHRI is relatively less sensitive than GNHRI and LHRI. LHRI exhibits relatively more sensitivity than the global policies to the inter-arrival time CV. The performance difference among three policies is marginal when the value of $C_{a}$ is small, but increases when $C_{a}$ equals 4 . In general, the performance of the three policies is not very sensitive to the inter-arrival time CV. The fact is that although the higher inter-arrival CV deters the receiver-initiated load distribution, the hierarchical organization, which reflects state of node accurately, allows receivers to acquire jobs from senders and to reduce the burden on the sender nodes. Note that there are six very lightly loaded nodes which could decrease the loads of those highly loaded nodes if the load sharing is effective as in LHRI, GNHRI and GRHRI policies. So the mean response time does not increase much even at high inter-arrival time CV.

\subsubsection{Sensitivity to Variance in Service Times}

This section compares the performance sensitivity of three hierarchical policies to the variance in service times. The parameter values used in this experiment are the same as those in the previous experiments, except that $C_{s}$ is altered to vary the variance in service times and $C_{a}$ is fixed at 1 for both classes of nodes. Figure 4.9 shows the results of the experiment. 


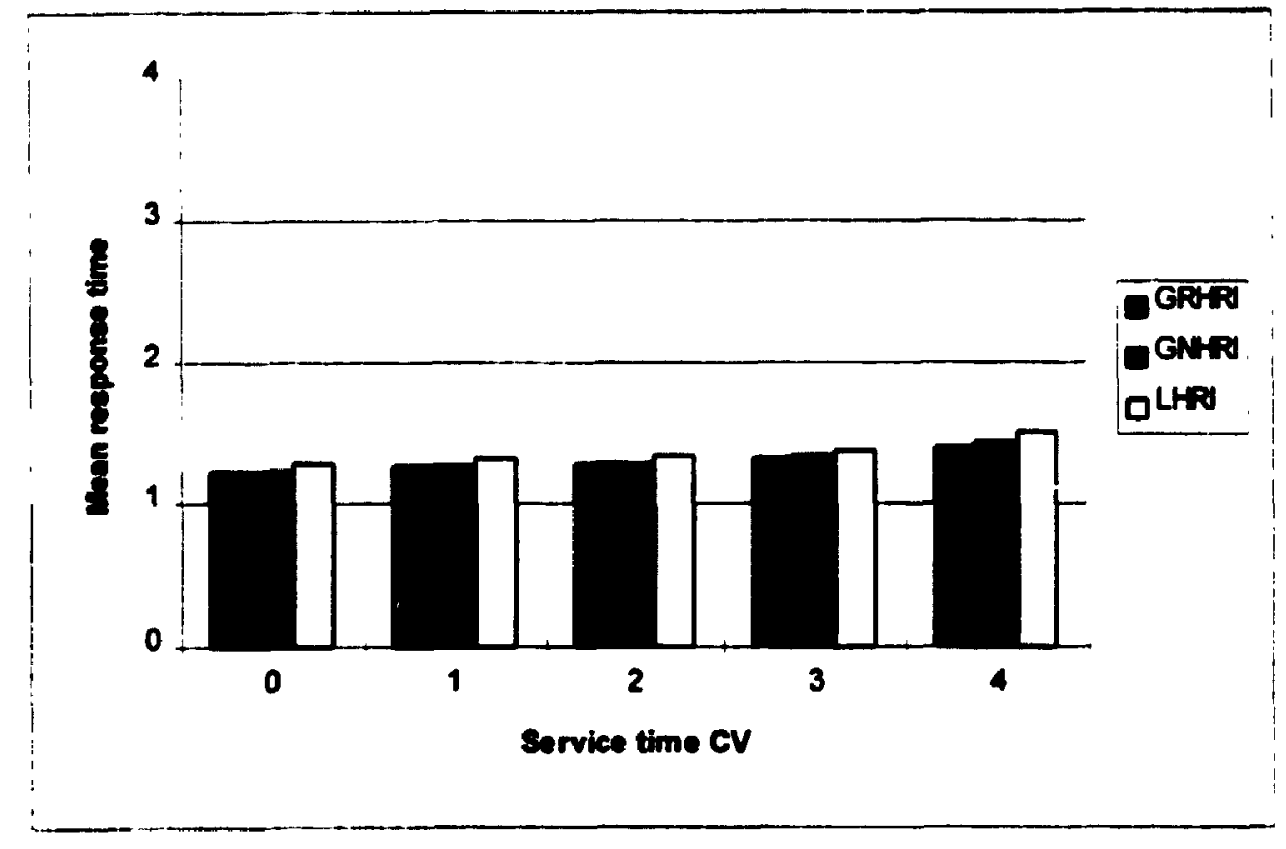

Figure 4.9 Performance sensitivity to service time $C V\left(N=32, N_{1}=6 N_{2}=26, C_{2}=1, C_{3}\right.$ is varied. $B=4$, $T_{h}=T_{1}=1, \lambda_{1}=0.1, \lambda_{2}=0.9, \mu_{1}=\mu_{2}=1, T_{\text {wat }}=1, T_{\text {false }}=0.2$, transfer cost is $1 \%$ of mean service demand)

The three policies are relatively less sensitive to the service time CV than to the inter-arrival time CV. These results are similar to those in the homogeneous system. GRHRI shows the least sensitivity to service time CV and LHRI shows the most sensitivity to such CV. The performance of GNHRI falls between the performance of LHRI and GRHRI. In general, these three policies are not sensitive to the service time CV.

\subsubsection{Impact of the Degree of Heterogeneity}

The impact of the degree of heterogeneity will be examined in this section. The offered load for Class I and Class II nodes is fixed at 0.1 and 0.9 respectively. The number 
of Class Il nodes, $\mathrm{N}_{2}$, is varied to change the degree of heterogeneity. The number of Class I nodes, $\mathrm{N}_{1}$, is given by $\mathrm{N}-\mathrm{N}_{2}$ where $\mathrm{N}$ is equal to 32 .

Figure 4.10 shows the mean response time as a function of the number of Class II nodes. As the number of Class II nodes (highly loaded nodes) increases, the response time increases because the system as a whole is subjected to higher workloads. GRHRI provides the best performance for all values of $\mathrm{N}_{2}$ tested. The performance difference between GRHRI and GNHRI and the performance difference between GRHRI and LHRI increase as the number of $\mathrm{N}_{2}$ nodes increases. This shows that GRHRI is relatively more effective in locating a sender with back tracking than the other two policies. The performance difference between GNHRI and LHRI is marginal.

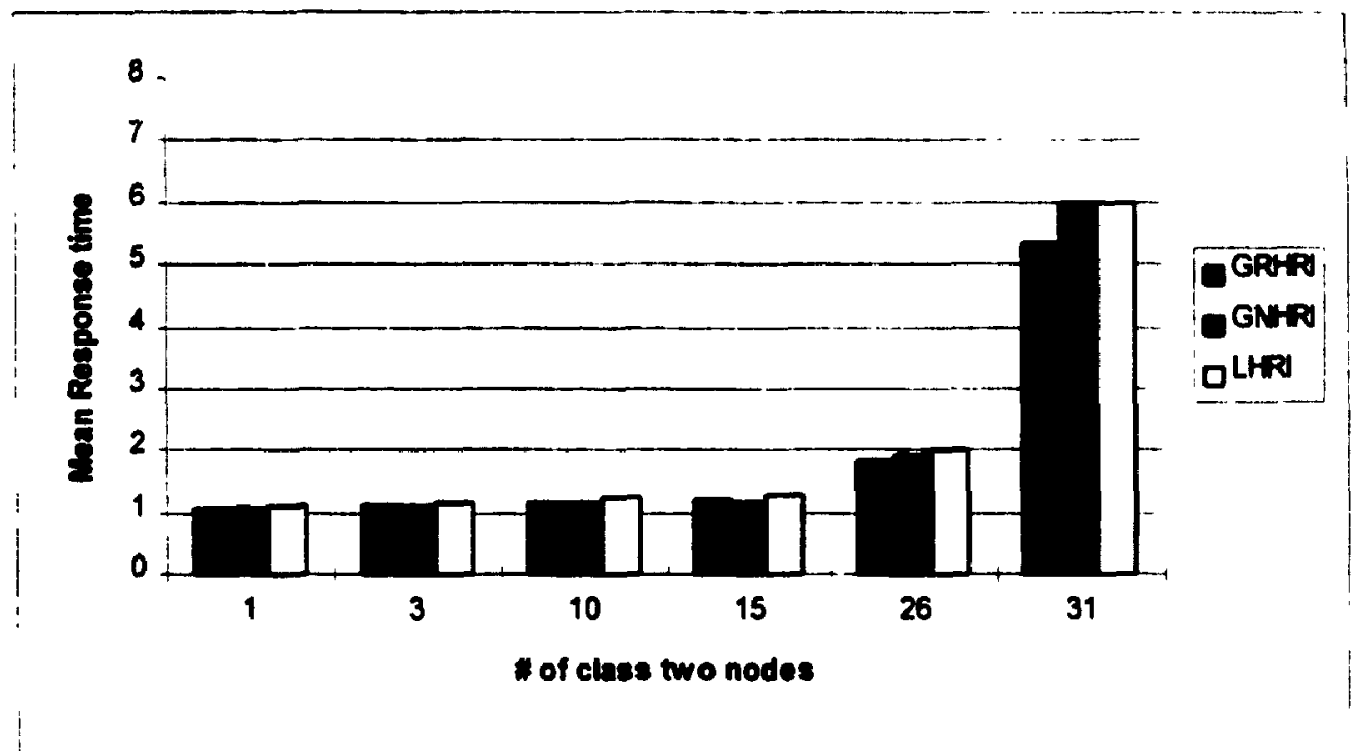

Figure 4.10 Performance sensitivity to the degree of heterogeneity $\mathbb{N}=32, N_{1}=N-N_{2}, N_{2}$ is varied, $C_{1}=C_{1}=4, \mu_{1}=\mu_{2}=1, \lambda_{1}=0.1, \lambda_{2}=0.9, T_{\text {wall }}=1, T_{\text {false }}=0.2, B=4$, transfer cost $=1 \%, T_{1}=T_{h}=1$ ) 


\subsection{Performance of Hierarchical Policies in Type II Heterogeneous Systems}

This section presents the performance of the hierarchical policies in Type II systems. Section 4.8.1 shows the mean response time as a function of offered load. Section 4.8.2 discusses the impact of the degree of heterogeneity.

\subsubsection{Performance as a Function of Offered Load}

The parameters in this experiment are the same as those used in Section 4.4.1. Figure 4.11 shows the perfi nance sensitivity to offered system loads. Note that at $90 \%$ load, the mean response time of all policies increases substantially from that at $80 \%$. The reason for this is that the faster nodes at such loads can no longer spare as much CPU time to smooth the workloads of slower nodes, as they are also very highly loaded.

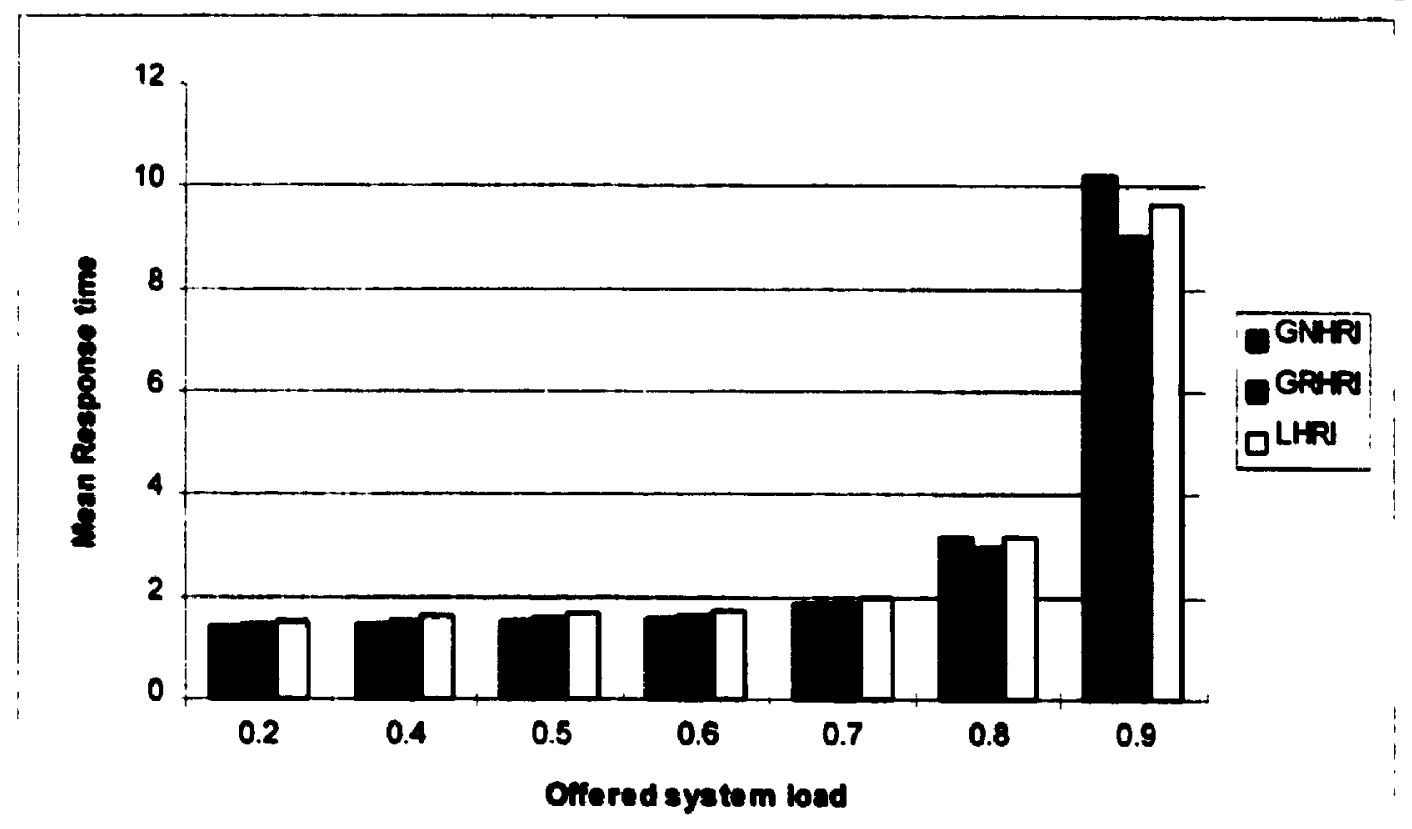

Figure 4.11 Performance as a function of system load $\mathbb{N}=32, N_{1}=N_{2}=16, \lambda_{1}=\lambda_{2} / 2, \lambda_{2}$ is varied, $C_{1}=C_{1}=4, \mu_{1}=0.5, \mu_{2}=1, T_{h}=T_{1}=1 B=4$ for all policies, $T_{\text {wait }}=1, T_{\text {fube }}=0.2$, transier cost $=1 \%$ ) 
The performance of GNHRI is marginally better at low to moderate loads than the other two hierarchical policies. At low to moderate loads. the performance difference between GRHRI and the other two hierarchical policies is marginal. At high system loads GRHRI performs (marginally) better than the other two policies. The performance of GNHRI at $90 \%$ load is worse than that of LHRI. This is because the net effect of "falsesender" scenario is larger than the effect of giving higher priority to local load sharing in LHRI. At such system load, receivers' requests are relatively easier to be filled even though senders may not be publicized as wide as possible.

\subsubsection{Performance Sensitivity to the Degree of Heterogeneity}

In this experiment the parameter values are set to the same as those described in Section 4.4.2. Figure 4.12 shows the mean response time as a function of the number of Class II nodes. All hierarchical policies are only slightly sensitive to the degree of heterogeneity at the given offered system load. This is because receiver-initiated load distribution is superior at higher system loads and the hierarchical organization allows all policies to effectively locate a sender for a receiver. Thus, the workloads of the senders can be shared with minimal delays. The result of this experiment shows that GRHRI exhibits least sensitivity to the degree of heterogeneity. The performance difference between GNHRI and LHRI is marginal. For some values of $N$ (e.g., 5 and 10), LHRI produces lower mean response time than GNHRI, but for other values GNHRI is better. This variation in performance is due to the effect of giving a higher priority to local sharing and the effect of the drawback of a "false-sender" scenario. The "false-sender" effect on 
GNHRI is greater than that on LHRI, since giving priority to local sharing reduces the number of "false-sender" cases. However, the effect of longer time delays in requesting jobs from senders in LHRI (because of higher priority in local load sharing) reduces the performance of LHRI. Thus, the performance depends on the net effect of these factors.

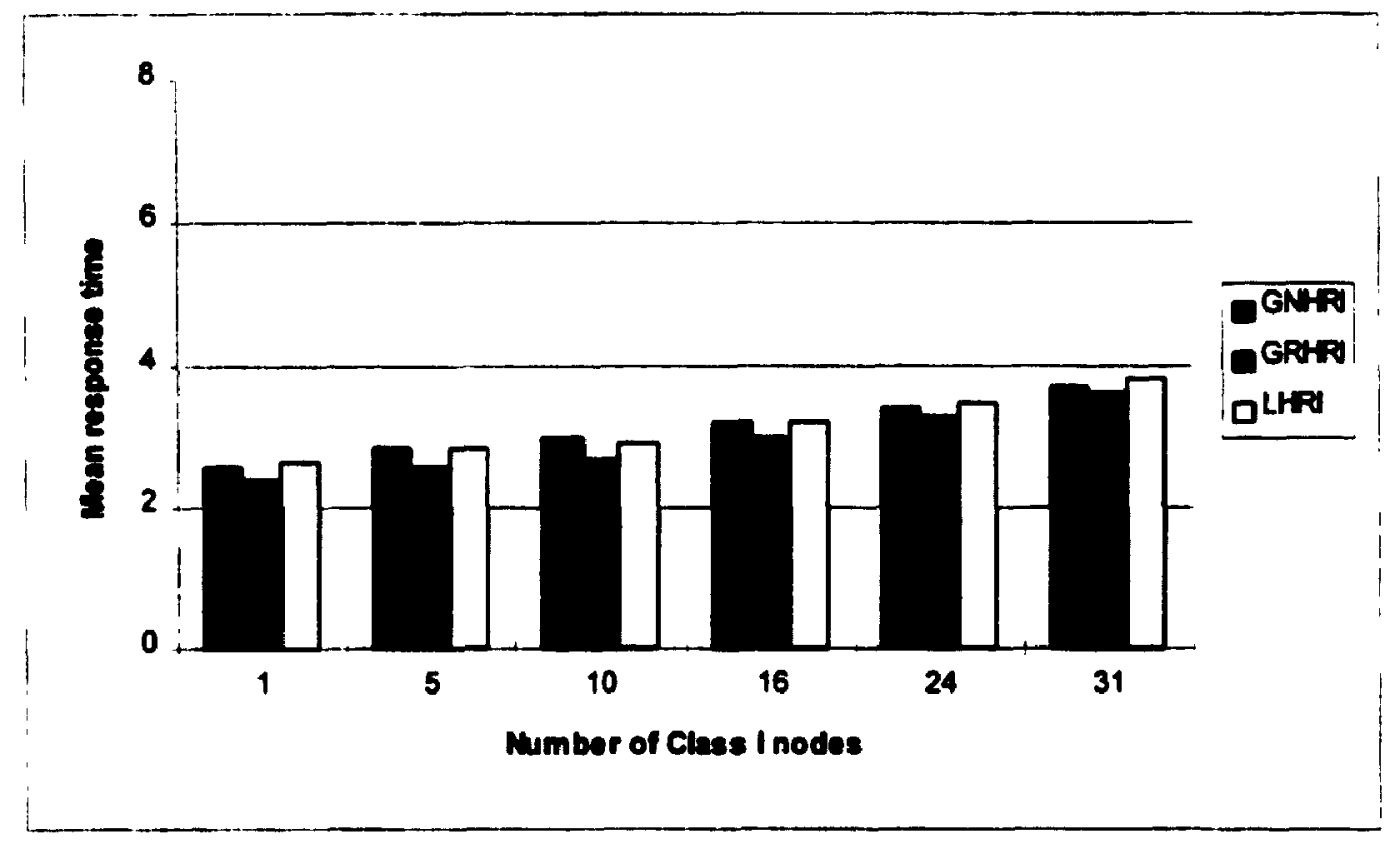

Figure 4.12 Performance sensitivity to degree of heterogeneity $\left(N=32, N_{1}\right.$ is varied, $N_{2}=N-N_{1}, \lambda_{1}=0.4$ $\lambda_{2}=0.8, \mu_{1}=0.5 \mu_{2}=1, C_{2}=C_{3}=4, T_{h}=T_{1}=1, B=4$, transfer cost $=1 \%$ of $\mu_{2}, T_{\text {wat }}=1, T_{\text {fule }}=0.2$ )

\subsection{Summary}

This section presents a brief summary of the results of the experiments. We have seen the performance of the five load sharing policies in both Type I and Type II heterogeneous systems. Then, we have also seen how the two variations of the local 
hierarchical policy perform in both types of heterogeneous systems. The following are the major observations:

Type I system:

1. SINCO, LHRI and SSYM perform significantly better than SI and RI over the whole range of system loads examined.

2. SINCO, LHRI and SSYM provide similar performance at low loads on Class II nodes but SINCO marginally outperforms the two policies at high loads on Class II nodes.

3. Over the whole range of system loads on Class II nodes, LHRI and SSYM provide similar performance. The performance difference is indistinguishable.

4. SINCO, LHRI and SSYM are relatively less sensitive to the variance in both service times and inter-arrival times than $\mathrm{SI}$ and $\mathrm{RI} . \mathbf{R I}$ is the most sensitive to the inter-arrival CV. However, $\mathrm{SI}$ is the most sensitive to the service time CV. Again the performance difference among SINCO, LHRI and SSYM in the sensitivity experiments is marginal. However, the impact of variance in inter-arrival times is larger than that of variance in service times on the three policies (for the parameter values chosen in the experiments).

5. For the hierarchical policies, GNHRI shows no noticeable improvement over LHRI but GRHRI performs marginally better than the other two hierarchical policies particularly at high system loads and at high variance in inter-arrival times and in service times. 
Type II system:

1. In such a system, the performance patterns are similar to those in a Type I system. SINCO is the best policy (especially at high system loads). The performance difference among SINCO and SSYM and LHRI is marginal (and in most cases are indistinguishable).

2. For the hierarchical policies, the performance of GRHRI is marginally better than that of LHRI and GNHRI at high system loads. At low to moderate loads, the performance difference among the hierarchical policies is negligible. The performance improvement of GNHRI over LHRI is insignificant, in some extreme cases (at very high loads) LHRI performs marginally better than GNHRI. 


\section{CHAPTER V \\ CONCLUSIONS}

\subsection{Summary}

Chapter 1 presented the reasons for considering load sharing in distributed computer systems and introduced issues related to load sharing. As well, the classes of load sharing policies, load characterization, and the components of typical load sharing policies are discussed.

Chapter 2 presented a survey of dynamic load sharing policies and the goals and motivation for this thesis.

Chapter 3 described the simulation models, which are used to evaluate the performance of six load sharing policies in homogeneous systems. The results of the simulation experiments are also presented in this chapter. Two vanations of the local hierarchical policy along with the performance implications are also discussed. A summary of results is given at the end of the chapter.

Chapter 4 reported the performance of five load sharing policies in both types of heterogeneous systems. The results are summarized at the end of the chapter.

\subsection{Contribution of the Thesis}

The main contributions of the thesis are in Chapter 3 and 4. In Chapter 3, six load sharing policies are evaluated in a homogeneous distributed system model. The 
performance of two variations of the local hierarchical load sharing policy is studied as well. The results from the simulation experiments show that, in general, the single coordinator policy (SINCO), local hierarchical receiver-initiated policy (LHRI) and stable symmetrically-initiated adaptive policy (SSYM) perform equally well. In addition, they are less sensitive to variance in both service times and inter-arrival times than receiverinitiated and sender-initiated policies. The results have also shown that the global recursive hierarchical receiver-initiated policy (GRHRJ) outperforms LHRI, particularly at moderate to high system loads. However, the globai non-recursive hierarchical receiver-initiated policy shows no significant improvement over local hierarchical policy. This concludes that the difference in the information maintained itself cannot provide improvement in performance unless recursive method is used together with global sharing strategy in which the existence of a sender is publicized as widely as possible.

In Chapter 4 the performance of five load sharing policies in two types of heterogeneous distributed systems is studied. The policies are SINCO, LHRI, SSYM, SI and RI. Moreover, the three hierarchical receiver-initialed load sharing policies are also evaluated under the same workload and system models used to compare the performance of the five load sharing policies. The results show that SINCO, LHRI and SSYM yield much better performance in heterogeneous systems than SI and RI. These three policies exhibit less sensitivity to variance in service times and inter-arrival times than $\mathrm{RI}$ and SI. The results also indicate that $4 H R I$ and SSYM are the best alternatives to SINCO. Finally, this thesis has demonstrated that job transfers initiated by receivers may not pose 
a performance problem as long as only non-executed jobs are considered and FCFS scheduling is used (e.g., the hierarchical policies).

\subsection{Further Research}

In this thesis, the performance of six load sharing policies in a homogeneous system and the performance of five load sharing policies in two types of heterogeneous systems are studied. However, the workload model assumes only CPU intensive jobs. Further research is needed to investigate the impact of other types of jobs such as parallel jobs, $1 / O$ intensive jobs and memory intensive jobs.

A parallel job is usually composed of many tasks. It may pose a performance problem on the communication network of a distributed system if the tasks of a single job are executed in different nodes and require frequent communication. Moreover, if the transferred tasks of a parallel job communicate frequently with each other, then the response time of the parallel job may be significantly higher than that if all tasks of the job are executed locally. This is because the cost of local communication is much cheaper than that of remote communication. Thus, a more complex selection policy is needed to incorporate to the LHRI and SSYM policies for parallel jobs in order to achieve high performance.

An I/O or memory intensive job may only be executed on certain nodes in a heterogeneous distributed system since nodes may have different capacities. We need to design a new model in which nodes have different capacities rather than just different 
processing capacities to evaluate the performance of load sharing policies when I/O or memory intensive jobs are considered. The LHRI and SSYM policies may require modifications in order to yield better performance. 


\section{REFERENCES}

[Ben94]

K. Benmohammed-Mahieddine, P.M. Dew, and M. Kara, "A Periodic SymmetricallyInitiated Load Balancing Algorithm for Distributed Systems," IEEE Int. Conf. Dist. Computing Systems, 1994, pp. 616-623.

[Dan95a]

S. P. Dandamudi, "Performance Impact of Scheduling Discipline on Adaptive Load Sharing in Homogeneous Distributed Systems," IEEE Int. Conf. Dist. Computing Systems, 1995, pp. 484-492.

[Dan95b]

S. P. Dandamudi, "The Effect of Scheduling Discipline on Adaptive Load Sharing in Heterogeneous Distributed Systems," Tech. Report 1995, School of Computer Science, Carleton University, Ottawa, K1S 5B6 Canada .

[Dan96]

S. P. Dandamudi and K.C. Michael Lo, "Hierarchical Load Sharing Policies for Distributed Systems," Tech Report SCS-96-1 1996, School of Computer Science, Carleton University, Ottawa, K1S 5B6 Canada.

[Dik89]

P.Dikshit, S.K. Tripathi, and P.Jalote, "SAHAYOG: A Test Bed for Evaluating Dynamic Load-Sharing Policies," Software-Practice and Experience, Vol. 19, No.5, May 1989, pp. 411-435.

[Dou91]

Fred Douglis and John Ousterhout, "Transparent Process Migration: Design Alternatives and the Sprite Implementation," Software-Practice and Experience, Vol. 21(8), pp. 757. 785 August 1991.

\section{[Eag86a]}

Derek L. Eager, Edward D. Lazowska and John Zahorjan, "A Comparison of ReceiverInitiated ans Sender-Initiated Adaptive Load Sharing," Performance Evaluation, Vol.6, No.1, Mar 1986, pp.53-68.

\section{[Eag86b]}

Derek L. Eager, Edward D. Lazowska and John Zahorjan, "Adaptive Load Sharing in Homogeneous Distributed Systems," IEEEE Trans. Software Eng., Vol. 12, No.5, May 1986, pp.662-675. 
[Eag88]

Derek L. Eager, Edward D. Lazowska and John Zahorjan, "The Limited Performance Benefits of Migrating Active Processes for Load Sharing," ACM Sigmetrics Conf., 1988, pp. 63-72.

[Kob81]

H. Kobayashi, Modeling and Analysis: An Introduction to System Performance Evaluation Methodology, Addison-Wesley, Reading 1981.

[Knu91]

Thomas Knuz, "The Influence of Different Workload Descriptions on Heuristic Load Balancing Scheme,” IEEE Trans. Software Eng., Vol. 17, No. 7, July 1991, pp. 725-730.

[Kru87]

Philip Krueger and Miron Livny, "The Diverse Objectives of Distributed Scheduling Policies," IEEE Proc. Seventh Int'l Conf. Distributed Computing Systems, 1987, pp. 242-249.

[Kru88]

Philip Krueger and Miron Livny, "A Comparsion of Preemptive and Non-Preemptive Load Distributing," Eighth Int'l Conf. Distributed Computing Systems, 1988, pp. 123130.

[Lel86]

W. E. Leland and T. J. Ott, "Load Balancing Heuristics and Process Behavior," Proc. PERFORMANCE 86 and ACM SIGMETRICS 86, 1986, pp. 54-69.

[Liv82]

Miron Livny and Myron Melman, "Load Balancing in Homogeneous Broadcast Distributed Systems," Proc. ACM Computer Network Performance Symp. 1982, pp. 47-55.

[Mir89]

Ravi Mirchandaney, Don Towsley, John A. Stankovic, "Analysis of the Effects of Delays on Load Sharing," IEEE Transactions on Computers, November 1989, pp. 1513-1525.

[Mir90]

Ravi Mirchandaney, Don Towsley and John A. Stankovic, "Adaptive Load Sharing in Heterogeneous Distributed Systems," Journal of Parallel and Distributed Computing, Vol. 9, 1990, pp.331-346

[Shi90] 
Niranjan G. Shivaratri, Philip Krueger, and Mukesh Singhal, "Two Adaptive Location Poliries for Global Scheduling Algorithms," IEEE Int. Conf. Dist. Computing Systems 1990, pp. 502-509.

[Shi 92]

Niranjan G. Shivaratri, Philip Krueger, and Mukesh Singhal, "Load Distributing for Locally Distributed Systems," IEEE Computer, December 1992, pp.33-44.

[Wan85]

Y. T. Wang and R. J. T. Morris, "Load Sharing in Distributed Systems," IEEE Transactions on Computers, 1985, C-34 (3), pp. 204-217.

[Zhou93]

Songnian Zhou, Xiaohu Zheng, Jingwen Wang and Pierre Delisle, "Utopia: aLoad Sharing Facility for Large, Heterogeneous Distributed Computer Systems," SoftwarePractice and Experience, December 1993, Vol. 23(12), pp.1305-1336. 

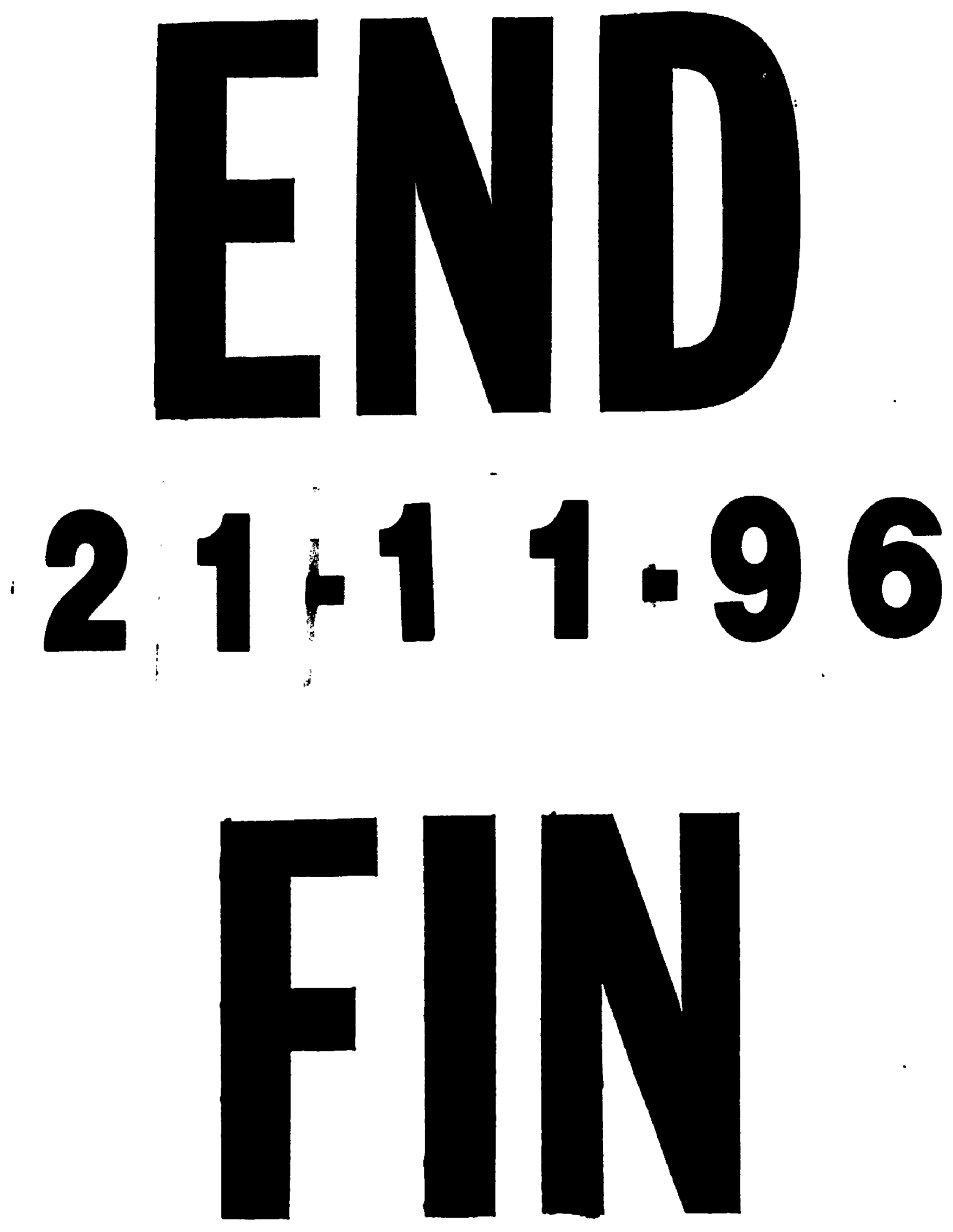